Original Research Paper

\title{
In Silico Prediction and Functional Characterization of Genes Related to Abiotic and Biotic Stresses in Chickpea (Cicer arietinum)
}

\author{
${ }^{1,2}$ Sukhdeep Kaur, ${ }^{2}$ Satendra Singh, ${ }^{1,2}$ Gitanjali Tandon, \\ ${ }^{1}$ Sarika Jaiswal, ${ }^{1}$ Mir Asif Iquebal, ${ }^{1}$ Anil Rai and ${ }^{1}$ Dinesh Kumar \\ ${ }^{I}$ Centre for Agricultural Bioinformatics, \\ ICAR-Indian Agricultural Statistics Research Institute, New Delhi-11012, India \\ ${ }^{2}$ Departments of Computational Biology and Bioinformatics, SHUATS, Allahabad-211007, India
}

\author{
Article history \\ Received: 09-11-2016 \\ Revised: 20-02-2017 \\ Accepted: 22-09-2017 \\ Corresponding Author: \\ Dinesh Kumar \\ Centre for Agricultural \\ Bioinformatics, ICAR-Indian \\ Agricultural Statistics Research \\ Institute, New Delhi-11012, \\ India \\ Email: dineshkumarbhu@gmail.com
}

\begin{abstract}
Chickpea (Cicer arietinum L.) is second largest grown legumes worldwide contributing $75 \%$ of total pulse production. It is a cool season legume crop and grown in tropical and subtropical areas. Due to drastic climatic changes, chickpea suffers from many biotic (blight and wilt) and abiotic (salinity, drought, cold) stresses that directly impact the growth and yield. In our study, we predicted and annotated the genes related to biotic and abiotic stresses. Total 20162 ESTs for salinity, 34346 for drought and 191 for cold stress were downloaded. For biotic stresses, viz., wilt and blight disease, 7866 and 56 ESTs were collected, respectively from public domain. All these ESTs were assembled into contigs and blast against protein nonredundant database. Each blast results were mapped to get the corresponding GO terms. Total 1631, 3133 and 13 contigs for salinity, drought and cold stress showed 1333, 2693 and 7 GO terms respectively, while 1144 contigs for Fusarium wilt and 6 contigs for Ascochyta blight disease showed 955 and 4 GO terms. These GO terms describe biological process, molecular function and cellular components of corresponding stresses. Remaining 298 (salinity), 440 (drought), 6 (cold), 189 (wilt) and 2 (blight) contigs were mapped to reference genome and further used for annotation using gene prediction methods and promoter analysis. This study provide insight to novel gene related to abiotic and biotic stress mechanism that can be further analyzed in molecular biology studies for breeding programs.
\end{abstract}

Keywords: Abiotic, Annotation, Biotic Stress, Chickpea, Gene Prediction

\section{Introduction}

Chickpea, a member of Fabaceae family also known as legume family is of varied nutritional and economic values. Studies show that chickpea seed comprise of $3-6 \%$ oil content, $40 \%$ carbohydrate and $20-30 \%$ crude protein (Jha et al., 2014). The climate requirement of chickpea is very favorable as it can easily grow in tropical and sub-tropical regions during winters that make it third highest produced legume crop in world and highest in Asia (Gaur et al., 2010). India is the largest producer of chickpea, comprising of $68 \%$ of global production as compared to America $(3.8 \%)$, Africa $(4.8 \%)$ and Europe $(0.9 \%)$. Total production of 13.1 million tons from an area of 13.5 million ha and a productivity of $9676 \mathrm{Hg} / \mathrm{ha}$ was recorded in year 2013 (FAOSTAT, 2012).

Comparative statistics of the global versus Indian chickpea production, area harvested and yield shows that India has major impact and contribution on global chickpea requirement. Various unfavorable conditions during life cycle of chickpea, especially during growing season causes approximately $50 \%$ yield loss each year and are increasing every year (Krishnamurthy et al., 2010; Ahmad et al., 2005; Varshney et al., 2010). Among the abiotic stresses, drought and salt stress are the major reasons for loss in production. Reports depicted 6.4 million tons yield loss due to abiotic stresses, out of 
which more than $40 \%$ occurred from terminal drought (Garg et al., 2016). Abiotic stress (drought, cold and salinity) contributes economic loss of approximately1.3 billion, 186 million and 354 million US dollars, respectively (Ryan, 1997). Among the various abiotic stresses affecting chickpea production, drought stress, particularly at the end of the growing season is a major constraint to chickpea production and yield stability in arid and semi-arid regions of the world. World's $20 \%$ of cultivable land is unable to provide quality yield due to increased soil salinity and high salt concentrated water used for irrigation (Flowers et al., 2010; Selvakumar et al., 2014). Other than abiotic stress, various biotic stresses also affect yield loss of 4.8 million tons (Ryan 1997). Supplementary Table S1 shows many biotic diseases listed in literature with their causative age.

Many fungal diseases damage this crop, of which Ascochyta blight disease caused by Ascochyta rabiei is very important, leading to severe damage. Sometimes, it results into complete yield loss due to blight formation during flowering and podding stage (Nene, 1982; Nene et al., 1996). Fusarium oxysporum pathogen infected seedlings show leaf drop and collapse due to browning and blackening of xylem (Kraft et al., 1994) causing wilt disease. Another rust causing fungus, Uromyces cicerisarietini infects the plant seedlings with visible round, brown spots causing leaf drop to death of plant (Stuteville et al., 2010). Phytoplasma are specialized bacteria that causes phyllody (Pallavi et al., 2012). Due to adverse climate and pathogen scenario, there is a great necessity to develop varieties resistant to such biotic and abiotic stresses.After the draft genome of chickpea, which was sequenced in 2013 (Varshney et al., 2013; Jain et al., 2013) ways to explore the novel and unpredicted stress genes expressing in biotic and abiotic stresses has been created.
There is a gap between potential and produced yield due to these stresses and need to fill by regulating corresponding genes. Although there are few studies available for identification of abiotic/biotic stress responsive genes using allele diversity approach (Roorkiwal et al., 2014) and microarray analysis (Mantri et al., 2007), but there are many unexplored genes that control stress responses and triggered stress responsive pathway by regulating corresponding transcription factors (Chen and Zhu, 2004). Available information and techniques for chickpea crop improvement needs more candidate genes for breeding strategies viz., gene pyramiding, marker assisted recurrent selection, multiline strategy.

In our study, efforts are made to understand the biology behind the stress conditions and characterization of genes that are getting expressed. In addition to functional characterization, computational analysis has been done to predict the novel candidate genes and their mapping on chromosome which can provide a good insight into complex abiotic/biotic stress tolerance pathways.

\section{Materials and Methods}

ESTs sequences related to abiotic and biotic stresses in chickpea were downloaded from NCBI-EST (http://www.ncbi.nlm.nih.gov/). Boolean search has been performed with the keywords like chickpea with salinity, drought, cold, fusarium wilt, Ascochyta blight and Chickpea rust. Figure 1 describes the flow of analysis for gene prediction and functional characterization. These ESTs were aligned and merged to reconstruct the gene sequences. Pre-processing of these ESTs were performed for repeat masking and cleaning, which was followed by assembly using EGassembler (Masoudi-Nejad et al., 2006). The generated contigs from EGassembler were considered for further analysis.

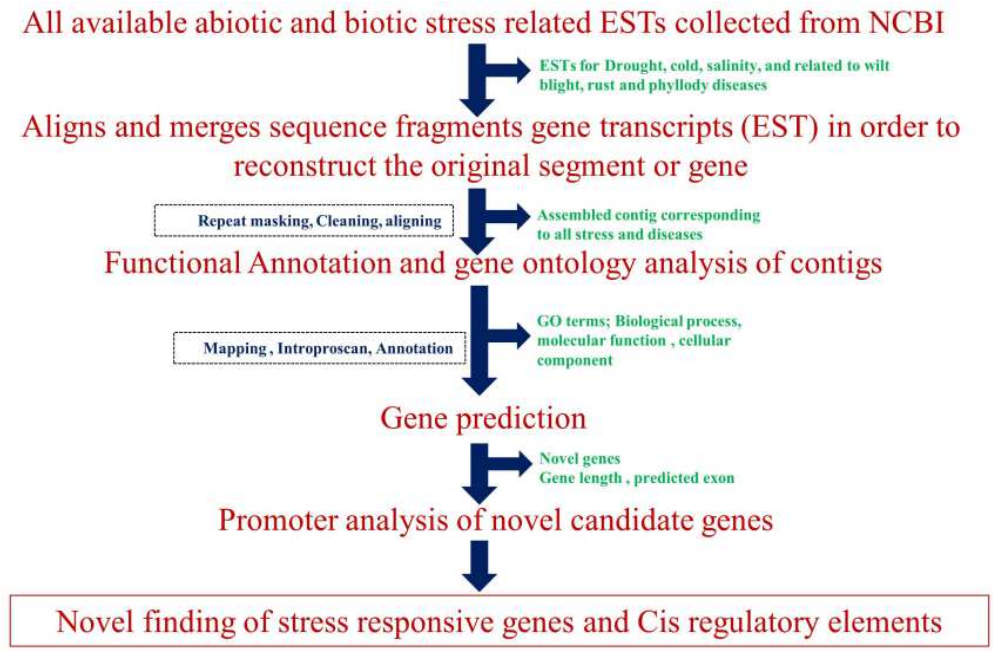

Fig. 1: Analysis work flow for gene prediction and functional characterization 


\section{Functional Characterization and Annotation}

Contigs assembled by EGassembler were annotated using Blast2GO Pro (Conesa et al., 2005) pipeline. Blastx (Altschul et al., 1990) was used to annotate the contigs for each stress individually. This was followed by mapping and InterProscan (Quevillon et al., 2005) to retrieve complete information of Gene Ontology (GO) and domains of annotated contigs searched against protein database for all six reading frames. Each GO term describes its involvement in molecular function, biological process and cellular component. Unannotated or uncharacterized contigs from all stress datasets were filtered out for gene prediction.

\section{Identification and Prediction of Unannotated Contig}

Filtered unannotated contigs were stored in fasta file format and subjected to ab initio gene prediction tool, FGENESH (Salamov and Solovyev, 1998) accessed through MolQuest version 2.4.5.1135 (http://www.molquest.com) for prediction of genes.

\section{Linkage Map and Promoter Analysis}

Predicted candidate genes were located on chromosomes and a linkage map has been created for disease/ stress findings using chickpea genomic web resource (http://www.nipgr.res.in/CGWR/home.php) (Kumari et al., 2014). All the predicted genes were searched in PLACE (Higo et al., 1999) database for identification of cis-elements, motifs, corresponding transcription factors and its description in other species.

\section{Results and Discussion}

In this study, chickpea ESTs related to abiotic stresses, like salinity, draught and cold downloaded were 20162, 34346 and 191, respectively. Among the biotic stresses in chickpea, wilt, blight, rust and phyllody diseases ESTs obtained were 7866, 56, 2 and 3 ESTs, respectively. A total of 1631,3133 and 13 contigs were generated by EGassembler for salt, drought and cold stress, respectively. Similarly, 1144, 6, 1 and 1 for contigs were generated for wilt, blight, rust and phyllody, stress, respectively (Table 1).

\section{Functional Annotation and Prediction of Candidate Genes}

Blast2Go Pro annotated total 1333, 2693 and 7 contigs related to salinity, drought and cold stress, respectively. Annotation was not obtained for contigs from rust and phyllody ESTs, while 955 and 4 annotation were recorded for wilt and blight related contigs, respectively. All these annotation were mapped to different $\mathrm{GO}$ categories i.e., biological process, molecular function and cellular components. Distribution of GO terms showed that $45 \%$ $47 \%$ were related to biological process, $33-36 \%$ to molecular functions and $19-20 \%$ to cellular components for drought, salinity, blight and wilt related contigs, respectively (Fig. 2).

Out of these, few contigs remained uncharacterized and did not map to any annotation. Selected uncharacterized contigs listed in Table 1 were subjected to gene prediction using FGENESH. Total 79 genes (salinity), 145 genes (drought), 27 (wilt) and 1 gene (blight) were predicted while, there is no gene was predicted for cold stress, rust and phyllody disease.

\section{Mapping of Candidate Genes and Identification of Cis-Regulatory Elements}

All predicted candidate genes were mapped to chromosomes. Mapping of genes shows that all genes were distributed randomly over all 8 chromosomes while many are still unallocated and placed on $U N$ chromosome. Major genes lie on chromosome 3, 5 for drought and salinity, while wilt genes are almost equally distributed on all chromosomes. Single gene predicted for blight disease is located on chromosome 4 (Fig. 3-6).
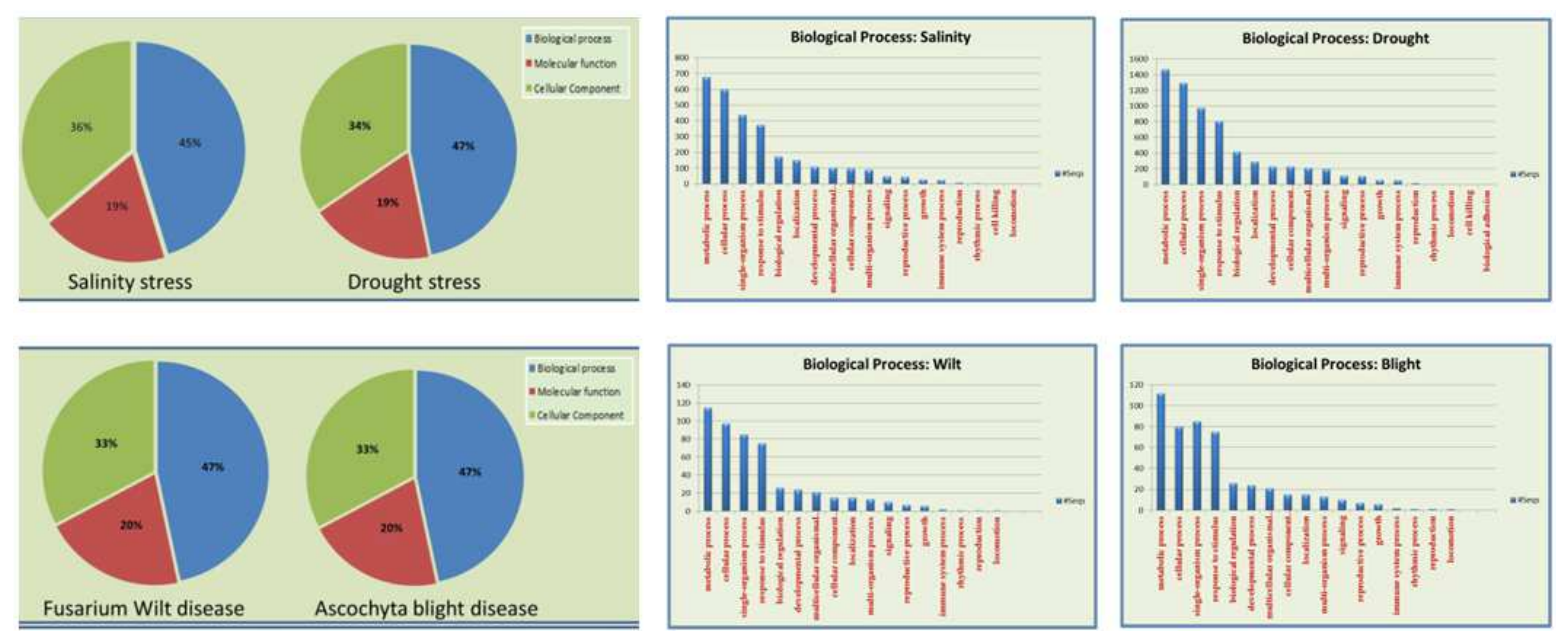

Fig. 2: Distribution of GO terms in abiotic and biotic stress related contigs and sequence distribution in biological process 
Sukhdeep Kaur et al. / American Journal of Bioinformatics 2018, Volume 7: 1.35 DOI: $10.3844 /$ ajbsp.2018.1.35

Table 1: Statistics of overall analysis of stress/disease related ESTs

\section{Abiotic Stress}

\begin{tabular}{llllll} 
Stress & $\begin{array}{l}\text { ESTs/Nucleotide } \\
\text { collected }\end{array}$ & $\begin{array}{l}\text { Contigs } \\
\text { generated }\end{array}$ & GO terms & Uncharacterized & $\begin{array}{l}\text { Novel gene } \\
\text { finding }\end{array}$ \\
\hline Cold & 191 & 13 & 7 & 6 & 0 \\
Salinity & 20162 & 1631 & 1333 & 298 & 79 \\
Drought & 34346 & 3133 & 2693 & 440 & 145 \\
Biotic Stress & & & & & \\
\hline
\end{tabular}

Biotic Stress

\begin{tabular}{|c|c|c|c|c|c|c|}
\hline Disease & Pathogen & $\begin{array}{l}\text { ESTs/ Nucleotide } \\
\text { collected }\end{array}$ & $\begin{array}{l}\text { Contigs } \\
\text { generated }\end{array}$ & GO terms & Uncharacterized & $\begin{array}{l}\text { Novel gene } \\
\text { finding }\end{array}$ \\
\hline $\begin{array}{l}\text { Fusarium } \\
\text { Wilt }\end{array}$ & $\begin{array}{l}\text { Fusarium } \\
\text { oxysporium }\end{array}$ & 7866 & 1144 & 955 & 189 & 27 \\
\hline $\begin{array}{l}\text { Ascochyta } \\
\text { blight }\end{array}$ & $\begin{array}{l}\text { Ascochyta } \\
\text { rabiei }\end{array}$ & 56 & 6 & 4 & 2 & 1 \\
\hline $\begin{array}{l}\text { Chickpea } \\
\text { Rust }\end{array}$ & $\begin{array}{l}\text { Uromyces } \\
\text { ciceris-arietini }\end{array}$ & 2 & 1 & 0 & 1 & 0 \\
\hline Phyllody & Phytoplasma & 3 & 1 & 0 & 1 & 0 \\
\hline
\end{tabular}

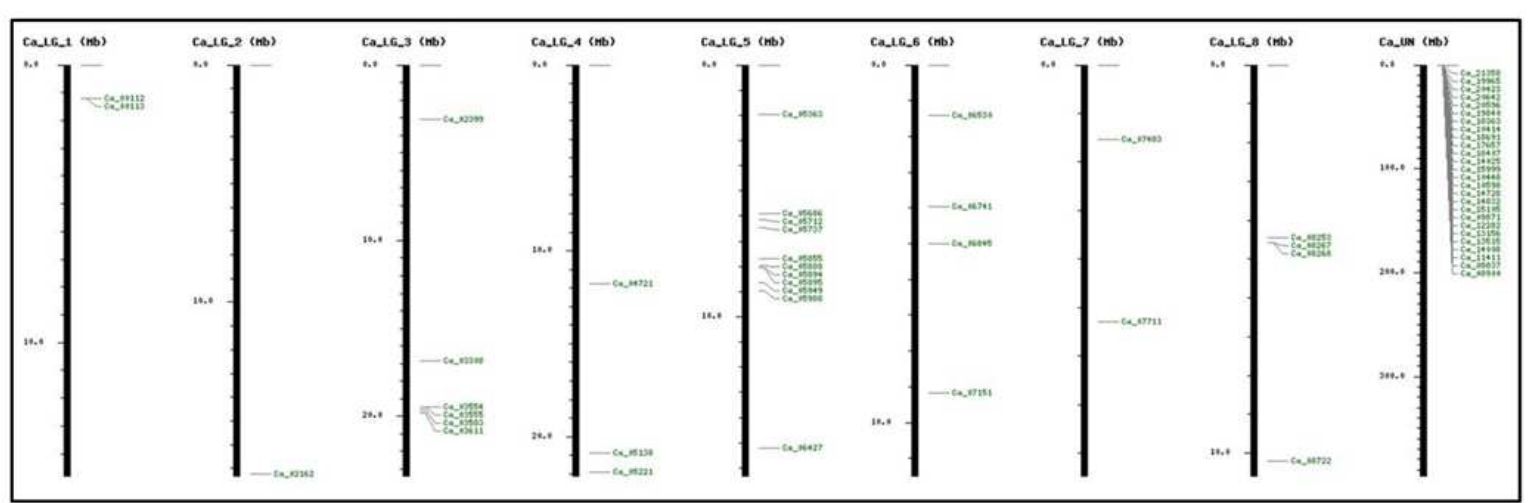

Fig. 3: Chromosomal mapping of drought related predicted genes

\begin{tabular}{|c|c|c|c|c|c|c|c|c|}
\hline $\begin{array}{l}\text { castas (nt) } \\
\cdots\end{array}$ & $\left.\right|_{-\infty} ^{c+1}$ & -cean & $\prod_{-\infty, \cdots n}^{c+16}$ & $\left.\right|_{-\infty} ^{c a+c .5}$ (nt) & 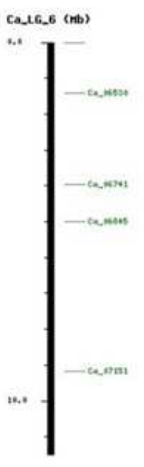 & 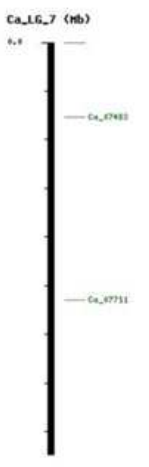 & |ates (nt) & (a.s. \\
\hline
\end{tabular}

Fig. 4: Chromosomal mapping of salinity related predicted genes

Total candidate genes located on chromosome shows that 39 genes are activated during exposure of both stresses while 20 genes are unique for salinity stress and
80 genes for drought stress (Fig. 7). It shows that these can be defense genes and playing major role in stress pathways (Supplementary Table S2). 


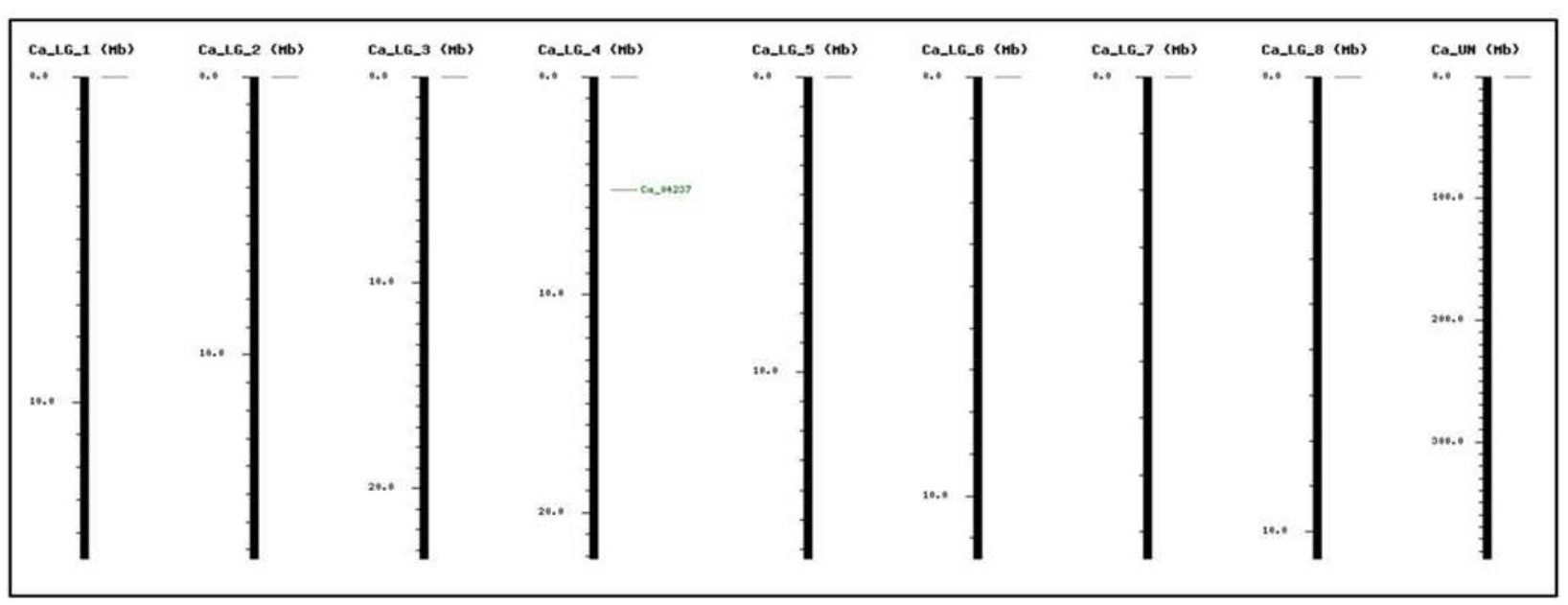

Fig. 5: Chromosomal mapping of Blight disease related predicted genes

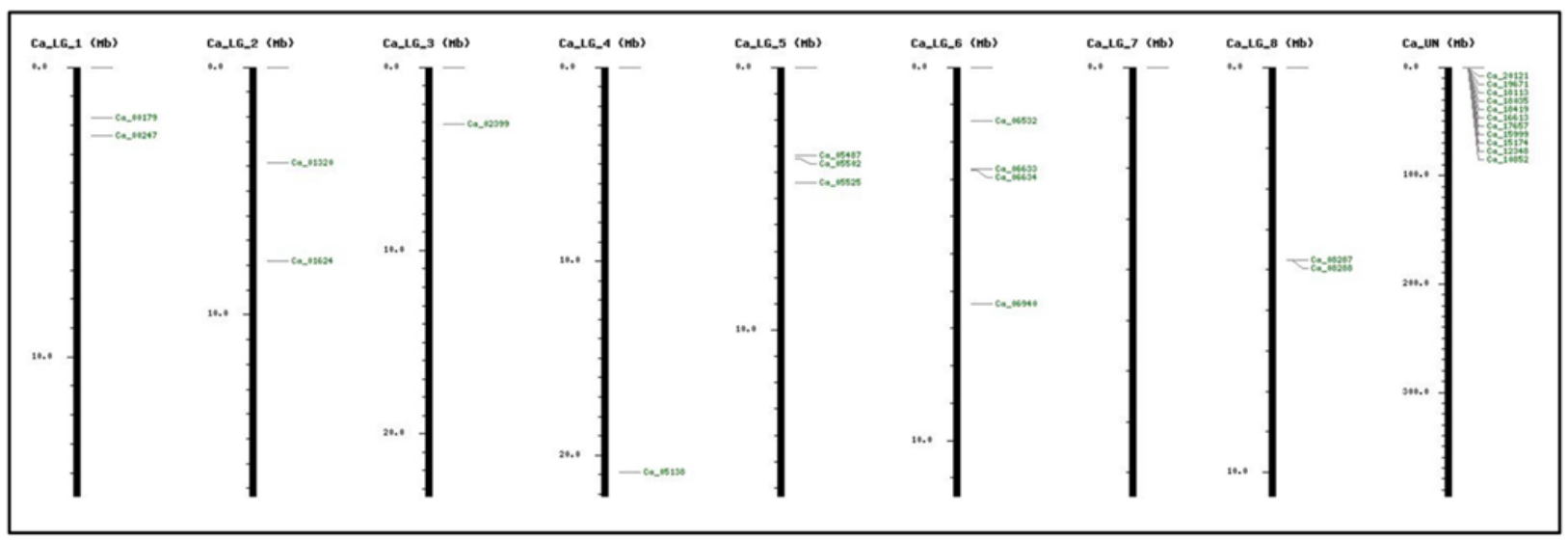

Fig. 6: Chromosomal mapping of Wilt disease related predicted genes

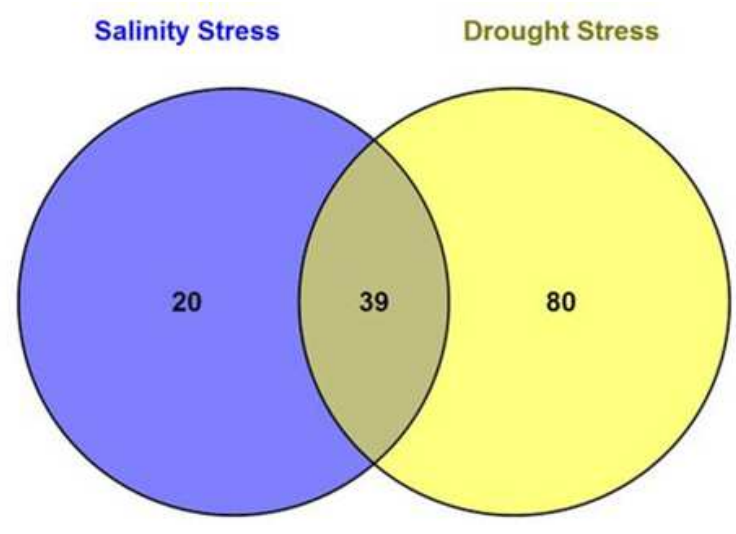

Fig. 7: Venn diagram depicting statistics of crosstalk genes of salinity and drought

Study also includes identification of cis-regulatory DNA elements that regulate the biological process in stress or disease conditions. To analyze this, we used
PLACE database with all predicted genes as query and identified cis-regulatory motifs. Analysis of cisregulatory elements describes the candidate gene expression and corresponding functional transcription factor. It can be suggested that genes which share common regulatory motifs are co-expressing and functioning in biological process in response to the corresponding biotic/abiotic stress. Cis-regulatory motifs and functioning transcription factor for each predicted candidate gene shows that in stress, transcription factors like DOF, bZIP, WRKY, RAV, ABRE and MYB are expressed majorly. These transcription factors are also reported in other crops viz., DOF in Chinese cabbage (Ma et al., 2015), bZIP in tepary bean (Phaseolus acutifolius) and common bean (P. vulgaris) (Rodriguez-Uribe and O'Connell, 2006) and other five legume genomes (Wang et al., 2015), ABRE and WRKY in soya bean (Li et al., 2005; Zhou et al., 2008).

DOF, which is plant specific transcription factor and known as DNA binding with one finger, contains 
conserved C2-C2 zinc finger and plays a significant role in plant growth and transcriptional regulation during stress conditions by aid of mobile proteins (Le Hir and Bellini, 2013; Yanagisawa, 2004). ABRE transcription factor participates in drought and high salinity tolerance in various crops by ABA signaling (Hossain et al., 2010). ABRE-binding bZIP transcription factor shows its presence in biotic stress. bZIPis reported as defense transcription factor that works during pathogen attacks in various crops like maize, Arabidopsis, rice and cotton and can assume same functioning in chickpea crop for stress management (Alves et al., 2013). WRKY transcription factor often functions in many stress responses simultaneously and participate in common signaling pathways. This property of WRKY makes it a good candidate for stress tolerance mechanism (Chen et al., 2012). Many predicted genes that were expressed during stress condition contain cis elements that provide binding sites to RAV which is known as Related to ABAinsensitiveViviparaous1. RAV transcription factor found to controls drought and salinity responses by participating in ABA independent stress pathway (Fu et al., 2014). Similarly MYB Family transcription factor plays role in various biological processes for $\mathrm{ABA}$ associated biotic and abiotic stress responses. It regulates functional genes to regulate functions as Phenylpropanoid metabolism, hormone responses, formation of cyclin -type B during plant defense reactions (Ambawat et al., 2013). Contig wise transcription factor abundance are listed in Supplementary Table S3.

The candidate genes of stress responsiveness can lead to crop improvement strategies viz., transgenic development, linked SNPs identification (Schena et al., 1995; Kudapa et al., 2013). These genes have been involved in abiotic/biotic stress tolerance in other crops like rice (Abbani et al., 2003), Arabidopsis (Kreps et al., 2002; Seki et al., 2002) whose information can be useful for chickpea also. These candidate genes can be selected for corresponding trait of interest on the basis of biochemical pathways and mutational analysis (Zhu et al., 2008).

\section{Conclusion}

Chickpea is economically very important crop and suffers from various biotic and abiotic stresses during its life cycle. In the present study, genomics approach is applied to predict genes related to drought, salinity, cold and disease caused by pathogen infections from ESTs available in public domain. In this study 1333, 2693 and 7 genes related to salinity, drought and cold stress respectively, were predicted, while 955 and 4 annotations were found for genes related to wilt and blight, respectively. These genes were found to be functional for DOF, bZIP, WRKY, RAV, ABRE and MYB transcription factors. The reported genes can be further used for candidate gene discovery required for Marker Assisted Selection (MAS) or gene pyramiding in crop improvement programme. Cis-regulatory elements and transcription factors study provides insight of their role in corresponding stress condition, whose validation is further warranted in Endeavour of improving chickpea productivity.

\section{Acknowledgment}

Financial support of Indian Council of Agricultural Research (ICAR), New Delhi is thankfully acknowledged. The grant of Fellowship to GT and SK by Indian Council of Agricultural Research (ICAR), New Delhi, Government of India and work supported by CABin grant $(\mathrm{F}>$ no. Agril.Edn.4-1/2013-A\&P,Project Code: 1004936) of ICAR-IASRI, New Delhi is duly acknowledged. Motivation and critical comment of HoD, Bioinformatics and Dean, Jacob School of Biotechnology, SHUATS, Allahabad is also thankfully acknowledged.

\section{Author's Contributions}

Sukhdeep Kaur, Satendra Singh and Anil Rai: Conceived this study.

Sukhdeep Kaur and Gitanjali Tandon: Participated in sample collection and data generation.

Sukhdeep Kaur, Mir Asif Iquebal and Sarika Jaiswal: Created the work-flow.

Sukhdeep Kaur: Performed data analysis.

Satendra Singh, Mir Asif Iquebal, Sarika Jaiswal and Kumar: Drafted the manuscript. All authors read and approved the manuscript.

\section{Conflict of Interests}

The authors declare that they have no competing interests.

\section{References}

Abbani, M., K. Maruyama, H. Abe, M. Khan and K. Katsura et al., 2003. Monitoring expression profiles of rice genes under cold, drought and high-salinity stresses and abscisic acid application using cDNA microarray and RNA gel-blot analyses ${ }^{1[\mathrm{w}]}$. Plant Physiol., 133: 1755-1767.

DOI: $10.1104 /$ pp.103.025742

Ahmad, F., P.M. Gaur and J. Croser, 2005. Chickpea (Cicer arietinum L.) In: Genetic Resources, Chromosome Engineering and Crop ImprovementGrain Legumes, Singh, R.J. and P.P. Jauhar (Eds.), CRC Press, Boca Raton, pp: 187-217. 
Altschul, S.F., W. Gish, W. Miller, E.W. Myers and D.J. Lipman, 1990. Basic local alignment search tool. J. Molecular Biol., 215: 403-410.

DOI: $10.1016 / \mathrm{S} 0022-2836(05) 80360-2$

Alves, M.S., S.P. Dadalto, A.B. Gonçalves, G.B. De Souza and V.A. Barros et al., 2013. Plant bZIP transcription factors responsive to pathogens: A review. Int. J. Molecular Sci., 14: 7815-7828. DOI: 10.3390/ijms14047815

Ambawat, S., P. Sharma, N.R. Yadav and R.C. Yadav, 2013. MYB transcription factor genes as regulators for plant responses: An overview. Physiol. Molecular Biol. Plants, 19: 307-321.

DOI: $10.1007 / \mathrm{s} 12298-013-0179-1$

Chen, L., Y. Song, S. Li, L. Zhang and C. Zou et al., 2012. The role of WRKY transcription factors in plant abiotic stresses. Biochim. Biophys. Acta, 1819: 120-128. DOI: 10.1016/j.bbagrm.2011.09.002

Chen, W.J. and T. Zhu, 2004. Networks of transcription factors with roles in environmental stress response. Trends Plant Sci., 9: 591-596. DOI: $10.1016 /$ j.tplants.2004.10.007

Conesa, A., S. Götz, J.M. García-Gómez, J. Terol and M. Talón et al., 2005. Blast2GO: A universal tool for annotation, visualization and analysis in functional genomics research. Bioinformatics, 21: 3674-3676. DOI: 10.1093/bioinformatics/bti610

FAOSTAT, 2012. http://faostat3.fao.org/home/index.html

Flowers, T.J., P.M. Gaur, C.L.L. Gowda, L. Krishnamurthy and S. Srinivasan et al., 2010. Salt sensitivity in chickpea. Plant Cell Environ., 33: 490509. DOI: 10.1111/j.1365-3040.2009.02051.x

Fu, M., H.K. Kang, S.H. Son, S.K. Kim and K.H. Nam, 2014. A subset of Arabidopsis RAV transcription factors modulates drought and salt stress responses independent of ABA. Plant Cell Physiol., 55: 1892-1904.

DOI: $10.1093 / \mathrm{pcp} / \mathrm{pcu} 118$

Garg, R., R. Shankar, B. Thakkar, H. Kudapa and L. Krishnamurthy et al., 2016. Transcriptome analyses reveal genotype- and developmental stage-specific molecular responses to drought and salinity stresses in chickpea. Scientific Rep., 13: 19228-19228. DOI: $10.1038 /$ srep 19228

Gaur, P.M., S. Tripathi, C.L.L. Gowda, G.V. Ranga Rao and H.C. Sharma et al., 2010. Chickpea seed production manual. Patancheru 502324 andhra Pradesh, India: International Crops Research Institute for the Semi-Arid Tropics. 28

Higo, K., Y. Ugawa, M. Iwamoto and T. Korenaga, 1999. Plant cis-acting regulatory DNA elements (PLACE) database: 1999. Nucleic Acids Res., 27: 297-300. DOI: 10.1093/nar/27.1.297
Hossain, M.A., J.I. Cho, M. Han, C.H. Ahn and J.S. Jeon et al., 2010. The ABRE-binding bZIP transcription factor OsABF2 is a positive regulator of abiotic stress and $\mathrm{ABA}$ signaling in rice. J. Plant Physiol., 167: 1512-1520.

DOI: 10.1016/j.jplph.2010.05.008

Jain, M., G. Misra, R.K. Patel, P. Priya and S. Jhanwar et al., 2013. A draft genome sequence of the pulse crop chickpea (Cicer arietinum L.). Plant J., 74: 715-729. DOI: 10.1111/tpj.12173

Jha, U.C., S.K. Chaturvedi, A. Bohra, P.S. Basu and M.S. Khan et al., 2014. Abiotic stresses, constraints and improvement strategies in chickpea. Plant Breed., 133: 163-178. DOI: 10.1111/pbr.12150

Kraft, J.M., M.P. Haware, R.M. Jimenez-Diaz, B. Bayaa and M. Harrab, 1994. Screening Techniques and Sources of Resistance to Root Rots and Wilts in Cool Season Food Legumes. In: Expanding the Production and use of Cool Season Food Legumes, Muehlbauer, F.J. and W.J. Kaiser (Eds.), Dordrecht, Kluwer Academic Publ, Netherlands pp: 268-289

Kreps, J., Y. Wu, H. Chang, T. Zhu and X. Wang et al., 2002. Transcriptome changes for Arabidopsis in response to salt, osmotic and cold stress. Plant Physiol., 130: 2129-2141. DOI: 10.1104/pp.008532

Krishnamurthy, L., J. Kashiwagi, P.M. Gaur, H.D. Upadhyaya and V. Vadez, 2010. Sources of tolerance to terminal drought in the chickpea (Cicer arietinum L.) minicore germplasm. Field Crops Res., 119: 322-330. DOI: 10.1016/j.fcr.2010.08.002

Kudapa, H., A. Ramalingam, S. Nayakoti, X. Chen and W. Zhuang et al., 2013. Functional genomics to study stress responses in crop legumes: Progress and prospects. Funct. Plant Biol., 40; 1221-1233. DOI: 10.1071/FP13191

Kumari, S., S. Pundhir, P. Priya, G. Jeena and A. Punetha et al., 2014. EssOilDB: A database of essential oils reflecting terpene composition and variability in the plant kingdom. Database, 2014: bau120-bau120. DOI: 10.1093/database/bau120

Le Hir, R. and C. Bellini, 2013. The plant-specific Dof transcription factors family: New players involved in vascular system development and functioning in Arabidopsis. Frontiers plant Sci., 4: 164-164. DOI: $10.3389 /$ fpls.2013.00164

Li, X.P., A.G. Tian, G.Z. Luo, Z.Z. Gong and J.S. Zhang et al., 2005. Soybean DRE-binding transcription factors that are responsive to abiotic stresses. Theor. Applied Genet., 110: 1355-1362. DOI: $10.1007 / \mathrm{s} 00122-004-1867-6$

Ma, J., M.Y. Li, F. Wang, J. Tang and A.S. Xiong, 2015. Genome-wide analysis of Dof family transcription factors and their responses to abiotic stresses in Chinese cabbage. BMC Genom., 31: 33-33. DOI: $10.1186 / \mathrm{s} 12864-015-1242-9$ 
Mantri, N.L., R. Ford, T.E. Coram and E.C. Pang, 2007. Transcriptional profiling of chickpea genes differentially regulated in response to high-salinity, cold and drought. BMC Genom., 2: 303-303. PMID: 17764573

Masoudi-Nejad, A., K. Tonomura, S. Kawashima, Y. Moriya and M. Suzuki et al., 2006. EGassembler: Online bioinformatics service for large-scale processing, clustering and assembling ESTs and genomic DNA fragments. Nucleic Acids Res., 34: W459-W462. DOI: 10.1093/nar/gk1066

Nene, Y.L., 1982. A review of Ascochyta blight of chickpea. Int. J. Pest Manage., 28: 61-70. DOI: 10.1080/09670878209370675

Nene, Y.L., V.K. Sheila and S.B. Sharma, 1996. A World List of Chickpea and Pigeonpea Pathogens. 5th Edn., Patancheru, ICRISAT.

Pallavi, M.S., H.K. Ramappa, K.S. Shankarappa, K.T. Rangaswamy and W.A.R.T. Wickramaarachchi et al., 2012. Detection and molecular characterization of phytoplasma associated with chickpea phyllody disease in south India. Phytoparasitica, 40: 279286. DOI: $10.1007 / \mathrm{s} 12600-012-0221-9$

Quevillon, E., V. Silventoinen, S. Pillai, N. Harte and N. Mulder et al., 2005. InterProScan: Protein domains identifier. Nucleic Acids Res., 33: W116-W120. DOI: $10.1093 /$ nar/gki442

Rodriguez-Uribe, L. and M.A. O'Connell, 2006. A rootspecific bZIP transcription factor is responsive to water deficit stress in tepary bean (Phaseolus acutifolius) and common bean (P. vulgaris). J. Exp. Botanyu, 57: 1391-139. DOI: 10.1093/jxb/erj118

Roorkiwal, M., S.N. Nayak, M. Thudi, H.D. Upadhyaya and D. Brunel et al., 2014. Allele diversity for abiotic stress responsive candidate genes in chickpea reference set using gene based SNP markers. Abiotic Stress: Molecular Genet. Genom., 5: 91-91. DOI: $10.3389 /$ fpls.2014.00248

Ryan, J.G., 1997. A global perspective on pigeonpea and chickpea sustainable production systems: Present status and future potential. In: Recent Advantages in Pulses Research, Asthana, A.N., M. Ali (Eds.), Indian Society of Pulses Research and Development: IIPR, Kanpur, India, pp. 1-31.

Salamov, A. and V. Solovyev, 1998. Fgenesh multiple gene prediction program. httpd.genomic.sanger.ac.uk
Seki, M., M. Narusaka, J. Ishida, T. Nanjo and M. Fujita et al., 2002. Monitoring the expression profiles of 7000 Arabidopsis genes under drought, cold and high-salinity stresses using a full-length cDNA microarray. Plant J., 31: 279-292. DOI: 10.1046/j.1365-313X.2002.01359.x

Schena, M., D. Shalon, R.W. Davis and P.O. Brown, 1995. Quantitative monitoring of gene expression patterns with a complementary DNA microarray. Science, 270: 467-470.

DOI: $10.1126 /$ science. 270.5235 .467

Selvakumar, G., K. Kim, S. Hu and T. Sa, 2014. Effect of Salinity on Plants and the Role of Arbuscular Mycorrhizal Fungi and plant Growth-Promoting Rhizobacteria in Alleviation of Salt Stress. In: Physiological Mechanisms and Adaptation Strategies in Plants Under Changing Environment, Ahmad, P. and M.R. Wani (Eds.), pp: 115-144.

Stuteville, D.L., W.L. Graves, L.J. Dixon, L.A. Castlebury and A.M. Minnis, 2010. Uromyces ciceris-arietini, the cause of chickpea rust: new hosts in the Trifolieae, Fabaceae. Plant Disease, 94: 293-297. DOI: 10.1094/PDIS-94-3-0293

Varshney, R.K., C. Song, R.K. Saxena, S. Azam and S. $\mathrm{Yu}$ et al., 2013. Draft genome sequence of chickpea (Cicer arietinum) provides a resource for trait improvement. Nature Biotechnol., 31: 240-246. DOI: $10.1038 /$ nbt.2491

Varshney, R.K., M. Thudi, G.D. May and S.A. Jackson, 2010. Legume Genomics and Breeding. In: Plant Breeding Reviews, Janick, J. (Ed.), Wiley, USA, pp: 257-304.

Wang, Z., K. Cheng, L. Wan, L. Yan and H. Jiang et al., 2015. Genome-wide analysis of the basic leucine Zipper (bZIP) transcription factor gene family in six legume genomes. BMC Genom., 16: 1053-1053. DOI: $10.1186 / \mathrm{s} 12864-015-2258-\mathrm{x}$

Yanagisawa, S., 2004. Dof domain proteins: Plantspecific transcription factors associated with diverse phenomena unique to plants. Plant Cell Physiol., 45: 386-391. DOI: $10.1093 / \mathrm{pcp} / \mathrm{pch} 055$

Zhou, Q.Y., A.G. Tian, H.F. Zou, Z.M. Xie and G. Lei et al., 2008. Soybean WRKY-type transcription factor genes, GmWRKY13, GmWRKY21 and GmWRKY54, confer differential tolerance to abiotic stresses in transgenic Arabidopsis plants. Plant Biotechnol. J., 6: 486-503.

DOI: $10.1111 / \mathrm{j} .1467-7652.2008 .00336 . x$

Zhu, C., M. Gore, E.S. Buckler and J. Yu, 2008. Status and prospects of association mapping in plants. Plant Genome, 1: 5-20.

DOI: 10.3835/plantgenome2008.02.0089

\section{Supplementary Files}

Supplementary Table S1. List of various diseases of chickpea collected from literature Bacterial diseases 


\begin{tabular}{|c|c|}
\hline Bacterial blight & Xanthomonascampestrispv. Cassia \\
\hline Bacterial leaf spot & Burkholderiaandropogonis \\
\hline \multicolumn{2}{|l|}{ Fungal diseases } \\
\hline Acrophialophora wilt & Acrophialophorafusispora \\
\hline Alternaria blight & Alternariaalternata; Alternariatenuissima \\
\hline Aphanomyces root rot & Aphanomyceseuteiches \\
\hline Ascochyta blight & Ascochytarabiei \\
\hline Black root rot & Fusariumsolani \\
\hline Black streak root rot & Thielaviopsisbasicola \\
\hline Botrytis gray mold & Botrytis cinerea \\
\hline Collar rot & Sclerotiumrolfsii \\
\hline Colletotrichum blight & Colletotrichumcapsici; Colletotrichumdematium \\
\hline Cylindrocladium root rot & Cylindrocladiumclavatum \\
\hline Damping-off & Pythiumdebaryanum; Pythiumirregulare; Pythiumultimum \\
\hline Downy mildew & Peronospora sp. \\
\hline Dry root rot & Rhizoctoniabataticola \\
\hline Foot rot & Operculellapadwickii \\
\hline Fusarium root rot & $\begin{array}{l}\text { Fusariumacuminatum; Fusariumarthrosporioides; Fusarium } \\
\text { avenaceum; Fusariumequiseti; Fusariumsolanif.sp. eumartii }\end{array}$ \\
\hline Fusarium wilt & Fusariumoxysporumf.sp. ciceris \\
\hline Myrothecium leaf spot & Myrotheciumroridum \\
\hline Mystrosporium leaf spot & Mystrosporium sp. \\
\hline Neocosmospora root rot & Neocosmosporavasinfecta \\
\hline Ozonium collar rot & Ozoniumtexanum var. parasiticum \\
\hline Phoma blight & Phomamedicaginis \\
\hline Phytophthora root rot & Phytophthoracitrophthora; Phytophthoracryptogea \\
\hline Phytophthoradrechsleri; & Phytophthoramegasperma \\
\hline Pleospora leaf spot & Pleosporaherbarum; Stemphyliumherbarum \\
\hline Powdery mildew & Leveillulataurica; Oidiopsistaurica \\
\hline Rust & Uromycesciceris-arietini; Uromycesstriatus \\
\hline Sclerotinia stem rot & Sclerotiniasclerotiorum; Sclerotiniatrifoliorum \\
\hline Scopulariopsis leaf spot & Scopulariopsisbrevicaulis \\
\hline Seedling or seed rot & Aspergillusflavus; Trichotheciumroseum \\
\hline Stemphylium blight & Stemphyliumsarciniforme \\
\hline Trichoderma foot rot & Trichodermaharzianum \\
\hline Verticillium wilt & Verticilliumalbo-atrum; Verticilliumdahliae \\
\hline Wet root rot & Rhizoctoniasolani \\
\hline \multicolumn{2}{|l|}{ Viral diseases } \\
\hline Bushy stunt & Chickpea bushy stunt virus \\
\hline Distortion mosaic & Chickpea distortion mosaic virus \\
\hline Filiform & Chickpea filiform virus \\
\hline Mosaic & Alfalfa mosaic virus \\
\hline Narrow leaf & Bean yellow mosaic virus \\
\hline Necrosis & Lettuce necrotic yellows virus; Pea streak virus \\
\hline Proliferation & Cucumber mosaic virus \\
\hline Stunt & Bean leaf roll virus \\
\hline Yellowing & Pea enation mosaic virus \\
\hline \multicolumn{2}{|l|}{ Phytoplasmal diseases } \\
\hline Phyllody & Phytoplasma \\
\hline
\end{tabular}

Supplementary Table S2. Sequence information of novel predicted gene

\begin{tabular}{l} 
Wilt related genes \\
>Contig142 \\
ATGGTGAAAGATGCTGATGTCATCATTCCAAAGGCTGTTGATGCCATTAAGACTGTTGAAACCGTTGAGGGAAATGGTGGTCCTGGA \\
ACCATCAAGAAACTCACTTTCGTCGAGGGTGGACAAACCTTGTATGTGTTGCACAGAATAGAAGCAATTGATGAAGCAAATTTGGGA \\
TATAATTACAGCATAGTTGGGGGTGCAGGATTGTCAGAGACAGTGGAAAAGATATCATTTGAGGCAAAATTGTGTGAAGGCCCAAAT \\
GGAGGGTCCATTGGGAAGGTGAGCGTAAAATATCAAACCAAAGGAGATGCTAAGCCAATGAGAAGGAGGTTCAAGAAGGAAAGGC \\
AAAGGGTGATGCTCTTTTCAAGGCCATTGAGGGTTACGTTTTGGCCAATCCTAATTACAACTGA \\
>Contig224 \\
CGTGGTCGCGGCCGAGGTACGAGCTTGGTCGCCAGCCAGCTAACACCAAGCTATCAAGCAACAAAACTGTTAGACGCATTCGTGTTA \\
GAGGTGGCAATGTGAAGTGGAGAGCTCTCAGATTGGATACTGGAAATTTCTCATGGGGAAGTGAAGCTGTCACTCGTAAAACCCGTT \\
TGCTCGATGTGGTTTACAATGCTTCCAACAATGAGCTTGTGCGAACTCAGACCCTTGTGAAGAGTGCTATCGTGCAGGTTGATGCTGC \\
\hline
\end{tabular}


Supplementary Table S2. Continue:

TCCTTTCAAGCAGTGGTATCTTCAACACTACGGTGTTGAAATTGGAAGGAAAAAGAAAACTGCTTCCAAAAAAGATACACCAGAGGA GGCCGAAGCTGTTACAGAAGAAGCCAAAAAGAGTAACCATGTCCAAAGAAAATTAGAGACCCGCCAGAAAGAACGCCAGCTTGATT CCCACATTGAAGAGCAGTTTGGTGGTGGGCGTTTGCTTGCATGCATTTCATCTCGACCTGGTCAATGCGGCAGGGCTGATGGTTACAT TCTTGAAGGAAAAGAGCTTGAATTTTACATGAAGAAACTCCAGAGGAAGAAGGGCAAGGGTGCCGCTTGA

$>$ Contig246

ATGAGTCGCCATCCGGAAGTTCTGTGGGCGCAGCGGTCTGACAAGGTTTACCTGACTGTTGCTCTACCTGATGCCAAGGATGTTACTG TGAAGTGTGAGCCTCATGGTTTGTTTAGTTTCTCTGCTTCTAGGGTTCAACATGAATCTTACAGCTTTAGTTTGGAACTTTATGGATCA ATTGAACCTGGGGGTTGTAAAACTAAAACTAGCTCAAGAAACATACTATGCTCAATCCAGAAAGGACAAAGAGGTTGGTGGAAAAG GCTACTGAAGTCTGAAGAGAAACCTGCACCATACCTGAAGGTTGATTGGAATAAATGGTGTGATGAAGATGAAGAGTCAGATTATGA GTTGATATCTGACGACGATGGACGATTTGCTGGTGAAGATGATGAAAGCAGTGATGATGAAGGAATGTTATATCTCCCTGACTTGGA AAAGGCTAGAGGAAAGTAA

$>$ Contig250

ATGCGTTGCGCGATTCTCTATTCCTACGCTAAAGAACGTTCGGGTCCACTCAAACCCGGCGTCGACACCGTCGAAGAAGCTGTTAAGA CCGTCGTCGGTCCAGTCTACGAAAAATTCCACCAAGTCCCAGTCGAGCTTCTCAAATACGTCGATCGCAAAGTCGACGAATCCGTTTC TGAGATAGATCGCCACGTTCCTACTAACGTCAAGAAG

$>$ Contig283

ATGACCAAGGACGAAGCTGAGGAACTAGTCAAAAAGGCAGTTTCACATGCCATTGCTCGTGACGGTGCTAGTGGAGGAGTTGTCCGA ACAGTCATAATAAACTCAGAGGGAGTGACCAGGAATTTCTACCCTGGAGATCAACTGCCGATATGGCACGACGAACTGGAGTCTCAT AACTCATTGCTAGACATTCTTGGTGCCCCAGAGCCAATGAACATATGA

$>$ Contig357

ATGGTGATGCAAGTGTTGGGTGCTATTTGTGGTGCTGGTGTTGTGAAAGGGTTTGAAGGAAAAACAAGATTTGGTGATCTTAAAGGTG GTGCCAACTTTGTGAACCCTGGTTACACCAAAGGTGATGGACTTGGTGCTGAAATTGTTGGCACTTTCATTCTTGTTTACACCGTTTTC TCAGCCACTGATGCTAAGCGTAGCGCCAGAGACTCTCATGTCCCTATTTTGGCACCATTGCCAATTGGGTTCGCTGTGTTCTTGGTGCA TTTGGCTACAATCCCAATTACTGGAACCGGTATTAATCCAGCTCGTAGTCTCGGTGCTGCAATTATCTTCAACAAGGACCTTGGTTGGG ATGATCATTGGATCTTCTGGGTGGGACCATTTATTGGAGCAGCTCTGGCAGCTCTATACCACCAAGTCGTAATCAGAGCCATTCCCTTT AAGTCCAAGTAA

$>$ Contig364

ATGGCTATTGATAGTGCTCCAATGTATATAGCGGTAGCACAAGCAAAGAAAAGAGCCAAGAACCCTCCAGCTGCAGCCAATGACATC AGCAGCGATATGACAATGGCATTTATGGTTGCGGCCAGAAAGAAAAGCGTAAATACAAGCAATCCTGTAAGAGAAACAAAAGTAAC CGCCCCAACGGAGATAACGAGGATAGCGCGTAGGTGGCTACCGCGACGAGTCCATAGAAGAATATCACGTGCAGAATTTCTAGAAGC TTCTGGAAGAAGTGAAGCGTTGTCGGCGACGGAATTTTTGATCCGGCGAAGTAAGTTATTGTCATCTCCCGGTTCCGGAAAGAGTATG GCGGAAGCGAGACTGTTAAGGACCTCAGGCAAG

$>$ Contig431

GTATACTGGAAGCCATACAAGTATCCTTCACCACCACCTCCACCAGTTTATACTTCACCTCCTTACATTCCACACCCAGTTTATCACTC ACCTCCACCACCAGTTCATACTTCTCCCCCATACATTCCACATCCAGTTTACCACTCTCCACCACCAACTCCAACCAAGAAGCCTTACC TCTATTCCTCACCTCCTCCTCCTTACCACTATTAG

$>$ Contig442

GTTAATGGATTGTTCTTTGCAATTGGTCATGAGCCTGCAACTAAGTTTTTGGATGGTCAACTTGAACTTGACTCTGATGGATATGTTGT GACGAAGCCTGGGACTACGAAGACTAGTGTTGAGGGAGTTTTTGCTGCTGGTGATGTTCAGGATAAGAAGTATAGGCAAGCTATTAC TGCTGCTGGCACTGGGTGTATGGCGGCTTTGGATGCAGAACACTTCCTGCAAGGGATTGGTTTACAACAGGATAAGAGTGATTGA $>$ Contig444

ATGGAAGAGGATGTTGAAGATGAGGTAGCTGAAATCAAGGCAGCTCATTTTGAGGAATCAATGAAGTATGCACGAAGGAGT $>$ Contig444b

CAGCCCAGAGGGTTTGGATCTGAGTTTAGGTTTGCAGATTCCAGCTCTGGTGGCACTACTGCTGCTGCAGGGACATCTGACCCATTTA CATCTGCTGGAGGAGCTGATGATGATGATCTTTACAGTTAG

$>$ Contig533

ATGCCTCTTTTGCCATTGAATGAGGATCACGATGACGGCGACGATGATTATTATGATTATAACGATGAAGAAGAAAAGGTTAGAAAA GTACCATCTGCTTCATCGTCTTCTTCTTCCTCTTCATTGGCTGAGGATAAGAAACAAGAAGATGATCAAGTGGAGTTTCGTCAATCCTA TGCTGCTGAAATGAGGCTTAGATTGAGAAGTTTCAAGTCAAGGAGTTTTTCTCTTGCAGATCTGCAAGAACATGATGATGAGGAAGAT CATGATCGTTAA

$>$ Contig587

ATGGCGTTCATGAAGGGAAAGGCAGTGATTACCAAAGTGGCAGCTTTAGCGAGTTATGGCGTGTTACCAGGTGCCATGGTGGCTGCT CTCGTCTACTCTCCCCCTAATTACGCCGAAAAACACGCCCCCGCCACCTCCAATTAG

$>$ Contig609

ATGGCCTCTCTCGCACGTGGAGCCGCATCTCTAACTCGCGCTACTCTTTCCGCTTCCAAATCTCCGATTCAACTCATCCAACGTCGCGG TCTCGCCGGAGCTGCAGATCACCATGGTCCCCCTAAAGTTGCCTGCTGGAAAGACCCAATGAGTCTTCTAAATGGAAGGAAGAGCAC TTTGTGA

$>$ Contig663

ATGGCGAAGGGAAAGAAGCAACTGATGTCATCGGCACCATGGAGAGGCGAAGAGGACACCGAACAATTCCCAGACGCAAACCTCAA AGTTACCAGTCAACCTGATGGCACTTCAACTATGCATGTTCCTCGCTCCAAATCCAACAATCACAATCATGATGATGATTTAATTGAG ATTGATCCTGAACTTCGATACAGTTTCCAACGCAATTTTCAGTTTCTTCATAGAGTTTTTAGCATTGACACCGTGGTGAAACCTCTCCC ACCCGTCATGGCTTACAATGTCTCTCGCAACTTGAACTTCTTCACGCGCATTTTCACTCAATTCTTTGATCCCGAAGGCATTGCAGCTG CCCAGAAATCCATGGGCATAGGACAGGAAGACAGAGATCGCAGAGTTCGTTGA

$>$ Contig667

CGTGGTCGCGGCCGAGGTACCCATCACCTCCACCGCCACCATACAAATATCCATCACCACCACCTCCAGTTTACAAGTATAAGTCCCC TCCTCCACCTGTTTATTCACCACCTCCACCACATTATGTTTATGCTTCACCTCCTCCTCCGGTCTATTCCCCTCCTCCACCGCATTACAT CTATGCATCACCTCCTCCTCCTTACCATTAA

$>$ Contig677 
Supplementary Table S2. Continue:

GATTTCGAGCGGCCGCCCGGGCAGGTACGCGCTCAGCTCTCAACAGAGAGTGCCTTAGCACCAATCAGCTTACCGTCAGCGTGCGAA ACAACCCAATACTCGGTCCTCGTAGCGTCTCTCGACACTTGATATACACGAACATCGTTACCCTGCGAGGCTTCTCGAACAGCCTCGA TGACGTCGGTGTATTTGCCGTGAGAGTCCCAGTTGAGAGGATCAAGGATCCTGATGTCTGCCGAGTCGGGGGAGGAGTGGTTGATGA GTTTGGCGAATTCGGTCTCGCTGGGGAGACCATTCTTGCCCTCCCACTTGAGAGAAACCTCTTGGAAGGGCTCGTCTGCGTCGGATGT GTATACGGCGTTCTTGCACACGTCACTGATGGGTTTGGGGACTTGAGCGCCCTGGTCCTTTGTCTTGAAG

$>$ Contig691

ATGTCGGTTTCATCGAAAGGAGATTCGGTTCAAATTAGAGAGGTTTGGAACGATAATCTCGATGAAGAATTTGCATTGATCCGTGAAA TCGTTGACCAATTCAACTTTATAGCTATGGATACTGAGTTTCCTGGAGTTGTTCTTCGTCCTGTAGGGAATTTCAAAAACATCAACGAT TATAACTACCAGACTTTGAAGGATAACGTTGATATGTTGAAGCTGATTCAGTTAGGTCTCACTTTCTCTGATGAGAACGGAAACCTAC CTAACTGTGGTACCTGCCCGGGCGGCCGCTCGAAATCACTA

$>$ Contig704

GACATTGCAAACTCTGAGCTGCCTCCAACTCACCCAATCAGGCTTGGTCTTGCTCTGAACTTTTCTGTGTTTTACTATGAGATTCTCAA CTCTCCCGATAGGGCTTGCGGTCTTGCAAAACAGGCTTTTGACGAGGCTATCGCTGAATTGGATACATTGGGAGAGGAATCATATAAG GATAGCACTCTAATCATGCAACTTCTCCGTGATAACCTCACCCTTTGGACATCTGACATGCAGGACGATGGTGCTGATGAAATTAAAG AAGCTGCACCTAAAGGTGATGAACAGCAGTGA

$>$ Contig707

CGTGGTCGCGGCCGAGGTACTCCCTACTACTACAAATCCCCTCCACCACCAACGTCGTCTCCTCCACCTCCTTACCATTATGTGAGTCC TCCCCCACCTATAAAATCTCCTCCACCTCCATACCACTACACATCACCACCTCCTCCATCCCCATCTCCGGCTCCCACATACATTTACA AATCACCTCCCCCTCCAGTGAAATCACCGCCACCACCAGTTTACATTTATGCTTCCCCGCCACCACCAATCTACAAGTAA

$>$ Contig728

ATGTCGACCAAGCAAGGTGGAAAAGCTAAGCCTTTGAAGAAACCAAAGTCTGATAAAAAGGATTACGATGAGATTGACATGGCTAA CATCCAGAAGAAAAAGGATGAGGAGAAGGCTCTAAAGGAGTTAAAAGCTAAGGCACAACAGAAGGGATCTTTTGGAGGATCTGGGC TCAAGAAAAGTGGAAAGAAATAG

$>$ Contig730

ATGCTTCTTGAACTAATAACTGGACAAAGGGCTTTTGATCTAGCACGTCTTGCCAATGATGATGATGTCATGTTGCTTGATTGGGTTAA AGGACTTCTCAAAGACAAGAAGTTGGAAACACTGGTAGATGCAGAATTAAAGGGAGATTATGATGATGATGAGGTAGAGCAGTTAA TTCAAGTAGCTTTACTATGCACACAAGGGTCCCCTATGGAAAGACCAAAGATGTCCGAGGTGGTGAGAATGCTAGAAGGCGATGGTT TGGCCGAAAAATGGGAGCAATGGCAGAAAGAAGAGACGTACCTCGGCCGTGACCACGCTAAGCGCTAA $>$ Contig820

ATGGAAGGTTCGCGGTGCAGTCGCGTTAATGGAAGAGGATGGAGGTGTTGTCAACAAACACTTGTTGGTTACTCTCTTTGTGAACATC ATTTAGGGAAAGGAAGGTTGAGAAGTATGACAAGTGTTAGAAATAGATCTATTGGTATTGGATTTTCTAATACTACTACTACTACTAC TGCACCATCACTAACAAAGAATAATATTAATGCTTCATCTTCTAATTCTGATGTTGTTGAGAAGAGAATGATGAATTGTGCTGGGAAT GATGATGATAAGACGGATAATTTTGATGAGAAGAAGCAAAAGAAGAGAGTGAAGCTTGGAATGGTTAAAGCACGGTCTATAAGCAG CTTATTGGGACAGCCAGACAACAAGGTTGCTCCTGTAGGTGATGAGAATAACAAGTAA

$>$ Contig868AT

GTCGAAGATGAAAGGTTCCACGTTTGAAAAGGACGTGCCATGGAGAGCTACATCTTCATCTGTTAAGCCAATACCTAAAATTCATCAC TCTCCAATTCTTCGCGTTTCCCATACACCTTTTTCCGACTATGCAATTTCCATCATGAAGCATCCAGACCCTATAGGTGATGGTTTGGG TGATGAGGCTATTGTGGAGGCAGCTGGTCCTGAATGCATCATACCTGGTCAGATTACTCCCGTCAGACTACTTGGCCTCAAG $>$ Contig953

ATGGGTGGATTCCCCCACTATGGTGTTGTGAAGGAGGATTATCTGATGATCAAGGGAGGTGTTGTTGGTCCCAAGAAAAGGGTTATTA CTTTGCGTCAGTCTCTACTCAAGCAGACTTCTCGCAATGCCTTGGAGGATATCAAACTTAAGTTTATCGATACCTCTTCAAAGTTTGGG CATGGTCGCTTCCAGACAACACAAGAGAAACAGAAGTTCTATGGACGCCTCAAAGCTTAG

$>$ Contig1031

TTTGGTCACCTTCTCGGTCAACGACCAATTTCGCTGACTATGGTAGCAAAGAGGAAGCATTTTTTGATTCCGAACCTTGGATAGACTC AGATTGGGAAGATGATTTCTATAGAGACAATGGTGACTTTACACCATCTAGAGGTAACACTCCAATTCACCACAATTTTGGGACCCCA AATGTGAATAAAATCTTTTCTCAGAATAGAACTTCTGATTCTCCATCTGAATCATCATCACCAGATAAGAAAAAGAAACTGTTAGAGC TTTTCAAAGAAAGTCTCAAAGATAACACAAATGATGAAG

$>$ Contig1082

ATGGGTAAGAGGAAGGCAAAAACAAAGCCACCACCAAAGAAACGGATGGATAAGCTTGACACTGTCTTTAGTTGTCCTTTCTGCAAT CATGGTTCCAGTGTTGAATGCCGCATTGATATGAAGAACTTGATAGGGGAAGCTTCTTGCAGGATATGTCAAGAGAGCTTTAGCACTA CAATCACAGCTTTATCTGAGGCAATAGACATATACAGTGAATGGATTGATGAATGTGAACGGGTGAACACTGTAGAAGACGACGGTG CTTAG

$>$ Contig1112

GCAATCTTGTTAAGGTTAAGGGTTCATTCAAACTCGCTCCGGCTAAATCTTCTGTGGCTAAACCTAAAGCCGCTACTGCTCCCGCTGCT ACCAAGAAGGCTAAGGCTGTTACCAAGCCAGCCGCCAAGGCTGCTACCAAGCCCAAGGCTAAAGCTGTTGCGAAGCCTAAAGCAGC GGCAAAGCCCAAAGCTGCAACGAAGCCGAAAACGGCGGCGAAGCCAAAAGCGAAGACGGTTAAGACAACACCGGTGAAGAAGGCT GTTGCTAAGACAACGAAGAAGGTTCCTGTGAAGGGTGTGAAGAAGCCTAAGAGCGTTAAAACGCCGGTGAAGAAGGCTAAGAAATG A

\section{Blight related Genes}

$>$ Contigl

ATGGAGAGGATCATGAAAGCTCAAGCTCTGAGAGACAGCAGCATGAGTGGCTACATGTCAAGCAAGAAAACCATGGAAACCAACCC TGATAATGGCATTATGGAGGAGTTGAGGAAGAGAGCTGAAGCTGACAAGAATGATAAGTCAGTGAAGGACCTTGTGCTGCTGCTTTT TGAGACTGCCCTTTTGACTTCAGGTTTTAGCCTTGATGATCCAAACACCTTTGCTTCAAGGATTCATAGGATGTTGAAGTTGGGTCTTA GCATTGATGAGGATGTGACTGGTGGTGATGATGTTGATATGCCTCCATTGGAAGAGGATGGTGCTGAGGAGAGCAAGATGGAGGAA

\section{Salinity related genes}

$>$ Contig42 
ATGGGAGCACCTCCAAAGAGTGATGTTCCAATTATTACACCAAATGAGCTCACTGAAGCTGATGGTTTGTTGTTTGGTTTTCCAACAA GATTTGGAATGATGGCTGCACAATTCAAATCATTTTTGGATGCAACTGGAGGCCTATGGAACAAACAGTCACTTGCAGGAAAGCCTG CTGGCATTTTTTACAGCACTGGTTTTCAAGGAGGTGGCCAAGAGACTACACCGTTGACATTTATTACTCAACTTGTTCACCATGGAATG ATATTTGTGCCAGTTGGATACACATTTGGAGCTGGCATGTTTGAGATGGAGAAGGTGAAAGGTGGTTCACCTTATGGTTCAGGAACAT ATGCAGGGGATGGCTCAAGACAGCCTAGTGAGTTAGAATTGGCTCAAGCTTTTCATCAGGGGAAGTACTTTGCTGGAATTGCTAAGA AGCTCAAGGGACCACAATGA

$>$ Contig55

ATGATGAATATCGATCGTAATAACAACAATGAAGTCACTTCTCTAGAAGATAAAACAAGGGTAGCCCAGCCCAAAGCCAAGCATCCT ATCACCTTCTGCAAGCACCTGATAGTCTCTGCTCCACTCTCATCCGCAGCGGAAGCCGGACCAGGAGACGATGCATCAATACTAGGTC CAGGAGCACCTACCGGCGGTGCTGGAGGTCCTAACAACGACGGAAACGGTGCCGGAACACTGTGTTTCTTCCCTTTCTTTACCTTCTT CTTTGATTTACTCGGAGCTGGAGCGGGAACTCCAGCGGTTGGTGTAACTGCCGGTGCCGGAATTGTTGGTGCTGGTACAGGAGGAAA ATTTGCCGGAGGAGAGCTCACTGGAACAGGAACTGGTGGAGAACTCACCGGCACTGGTACCGGTGGTGAACTCACCGGAGTAACCGC CGGTGGTGACGATGCTGGAGGCTTAGCTACCGGAACTGGAGCAGCGGCATTTGAAGAAGGAGACGTAGCTGGTGTTGCAACTGCATT TGCAGCAGTAGGAGAAGAAGTTGGTGGTGCTGACGTGGGAGAAGATACAGGAGCGGGTGATTTAGGTTTGGAAGGGGTTGTGACGG GAGAAACAGTAGGAGTTGTTACGGTTGGTGATGTTGTCGGAGCCGATGAAGGAGATTGA

$>$ Contig71

ATGGCTGATTTTCAACAAAAACTTGATGCACTAAAGGGTCTTGTTGGTGTGCTAAATAGCATGGGTGATGATGGTCATGAAAATGGTG ATTTTCCTTCAACTCCACATAATTACCATCCTACCCCTCAGCCAGAAAGGAAACGATCCTCTGGAAAATATAGCAACACTGGCCACCA AAATATAAAAGGTTTAAGTAATCAAACCGGTTTTACTGAAGGCAATGCTAATGGAGCTATCAACTTTGGTGATTTAAAAATGTAA $>$ Contig125

ATGGAAATAGTTGGTGAGATTCAACGTGCCATGGCTAGAGTGTGGCTATGGGATGGAAGGTCTAAGGCCCCACCAAGACTTCTTGAT GGTATGGCCGAGTATGTTGCCGAGTTAGCCGGATTCCGCCGTGAAATATTCTCCGGCGGCTTCGGTGAGTCGCCGGAATGTGAAGTTG GCCGTGATTTATGGTGGGAAGATAAGGACCCTACTCATGTGGCACGTTTGTTGCATTATTGTGAAAATTTTAAGAAGGGGTTCATCCA ACGGCTGAATGAAGCTATGAGAGACACGTGGCATGATCGTATG

$>$ Contig139

TCTCCCCCACCACCGTACAAGTACCCATCACCTCCACCGCCACCATACAAATATCCATCACCACCACCTCCAGTTTACAAGTATAAGT CCCCTCCTCCACCTGTTTATTCACCACCTCCACCACATTATGTTTATGCTTCACCTCCTCCTCCGGTCTATTCCCCTCCTCCACCGCATT ACATCTATGCATCACCTCCTCCTCCTTACCATTAA $>$ Contig165

ATGACTCAGTACGGTATGATGCATTCAAAGCAGTGTAGGCCATCCAACTCTGGAGCAAACCTTGGTCGCAAACGGGTCGGGTTTAGCT TGTCTCCAGCGGGTATTATTGTTTTCGGATCTTTGGCGGTGGTGGCGACGGATGAGGAAGTGGCTTCCGTATTGGGCTTTAGATTGAC ATTATTTGCTGGGCCCATACCGAAGGAAAAGCACCATTTCTGGGCCTGCGAGATCTCAAAGTGGCTGAAGATAGACATAAACATTTTT ATTGCTAGGAAGAGGTAA

$>$ Contig167

ATGGAAACTCCAAGCATAGCCATTGAATTTGAAATAGATCCGGCAGCTCCGACATCGGGACCTGGTGCCGGTGCCGACGAAGGACTA AGGTCTTGTGCTGAGGCTACAGCGGCGAAGACTGCGACAAAAAAGAAGGAAAATAGCTTCATTGTAGTAGCTGATGATGCGATG $>$ Contig206

ATGGTTACTCACACAGCTGACTCTTCCTCTAATCACCACTCGCCAACCACTACTAATGCTGCCGCCATCAATAACCCTAATTACCCGCG AAAAAACTTACCTTCTCCGTGGATCAAGGTTGTTCGCGGTGCTGATTCCGAACCACTCAACCACCCATCGCCGCCATCGTCTTCTTCTT CGTCATCCATTGATTCTGGCAATTCCGATGCCGCCGCCGCTGCGTCTGCCGTAGATAACTCAAATGCTGACAAGAATAACAATGCGTG TAACCCTAAAAACGCTTGGAAGAATCCATCTAATGGTATTTCTGAT

$>$ Contig208

ATGAGTAATTACCAGAGACCACCTCAAGAACCTTACCCTCCACCAGGGTTTGGATCTCCTTACCTTCCACCATCACCCCATCTGCATC ACGAAGGCTACCCTCCACCACCACCACCGCATCACCACCGTCCGCCGCACGAATCCTACCAAGGTTATTTCGACAACGGTCATCCACC ACCGCCTCCTCCTCCGGCTCCGTCTCATTACCACTACCAACATATTGATCATCACCGTCATGATGATCACGGTTGTTCTTCCTTCTTCCG TGGCTGCTTGGCGGCGCTTTGTTGCTGTTGCGTGGTAGAGGAGTGTTGTTGCTGTTTCTGA

$>$ Contig219

CTCACCACCACCACCACCAACAAAGAAACCATACTACTACCATTCTCCACCACCACCAGTTTACTCACCTCCAAAACACCCTTACCAT TATCACTCTCCACCACCACCGAAGAAACCTTACAAGTATCCTTCACCACCACCACCAGTTCATCATGTTCACCCTAAACCCTATTACCA TTCCCCACCACCACCAAAAAAGCATTACAAATATTCCTTTCCTCCACCACCAGTGCAT

$>$ Contig281

ATGGCTTGCGTCGCCGGCACTGCCGCGAGATCGATATTCCGGTCATCCTCCGCCGCCCGCACCGCTTTCCGTATCGGTTCAGAAGCTA AAACAGCTCGTTCTCCTTTCCGAATCGCCTCCAATAAGCCTCTTTCGCAATCCGCACTCAGGTGTCCTGTTGAATTGAGCTTCTGTGTT GAATCGATGTTGCCGTATCATACGGCAACTGCTTTTGCGTTGATGACTTCAATGTTAGTAGTTTTTGAACGCAGATACGGTTGGCTTCC TGAAGGTAGCTAA

$>$ Contig282

ATGGAATCTCATGGAACATCATGGGCTGACCAATGGGAAAATGGCCCAGACCCTGTGAGTGGTTCCAACCAATCCAAAAAGAAGAGC AACAACATTCTAGGCAAGACCAAAACAGTGGCCTCCACTGGCGTCCAGAAGTTGAAAGAGGGTACCTCTGTTAGTCTCAAATGGATC AAAACCAAGTATAACAAAACCACCAACAAACATTAA

$>$ Contig288

ATGGCCTCTGTTCAGTGCTATAAAATCTGTGAACAAAGCTGCCAACAACACAAAACCCAGCAGCAACATGGTTCCTTAGGACAGAAG GTAACTGACTTGTTCAAAGGGCACCCCAATGAGGGAACACAAACCCAATACTGTAGCAAGAAAACTGAGGTGATTTCTCAGTCAGGA AATTTAGTTTCCAAAAGTGAAACTAAAAAATGCAACCAAACATTCAACAACAGTGGTGCTTCTAGTACCACCGTGAAATGCCAGGGG AGAAATAGGAGGCAGCACAAGAGAAACAACTTGTTCCAGAAGATCAAGGATGGCATATCAGGTCACAGCAGTGACAGTAGCAGTGA TGAGAGTGACAGTGACAATGAACATTGTCACAACAGGAAGAACTGA

$>$ Contig304

ATGGGGTCTCTAATGGCCTCTATCACTTTAACTCTTCTATTAGCCATACTATCTCTCACCACCTTCCCATCACAAATTTCAGCAAACAA TTACTTGTATTCCTCACCCCCACCACCCAAGGTATACCCCCCAGTCTCACCACCACCTAAGGTATACCCTCCAGTCTCACCACCACCTA 
AGGTATACCCTCCAGTTTCACCACCTCCTAAGGTGTACCCCCCAGTCTCACCACCACCTAAGGTGTACCCTCCAGTTTCACCACCTTAC CACTACTCCTCACCACCACCACCAGTTCATCAT

$>$ Contig374

ATGGGTGTGACAAAGGAACAAGTCGAGTCATCCTTGAACTCCAAACTTAACCCTTCCCATATCGAAGTACTGGATACATCTGGAGGGT GTGGTGCAAGTTTTGTAGTTGAGATTGTGTCTGAACAATTTGAAGGGAAAAGGCTATTGGAAAGGCATAGAATGGTGAATGCTGCCTT AGAGGAGGAAATGAAAGAGATTCATGCACTTTCTGTAAAGAAAGCTGTAACCCCAGAACAGTGGAAAAAACTGCAAGAGTCCAACC AATCAAATCCTGCTGCTTAG

$>$ Contig395

TGTGGTAGAGCAATTGGTGTTGATTTGCTTAATAACCCAGACCTTGTTGCTACCGACCCTGTTATTTCCTTCAAGACCGCTATATGGTT CTGGATGACCCCACAGTCACCCAAGCCATCCTGTCATGATGTTATCACCGGACGATGGAATCCTTTTACTTTTGATCGTGCGGCTGGTA GGCTTCCTGGTTATGGTACTGTTACCAACATCATTAACGGTGGACTTGAATGCGGTAGAGGACATGATAGCAGAGTTCAAGATCGGAT CGGTTTTTACAAGAGATACTGTGACATCTTTGGTATTGTTTACGGAGATAACATTGACTGTGCTTTTCAAACACCATTTGCTTTTTCTCT ATCCTTTTCATCATTGCTGCTCCAATCCAAACCATCCACACCTAGTCAGTAG $>$ Contig411

CCATACAAATATTACAAGAAGCCATACAAGTATCCTTCACCACCACCTCCACCAGTTTATACTTCACCTCCTTACATTCCACACCCAGT TTATCACTCACCTCCACCACCAGTTCATACTTCTCCCCCATACATTCCACATCCAGTTTACCACTCTCCACCACCAACTCCAACCAAGA AGCCTTACCTCTATTCCTCACCTCCTCCTCCTTACCACTATTAG

$>$ Contig437

TTTTGTGGCTAAACCTAAAGCCGTTACTGCTCCCGCTGCTACCAAGAAGGCTAAGGCTGTTACCAAGCCAGCCGCCAAGGCTGCTACC AAGCCCAAGGCTAAAGCTGTTGGGAAGCCTAAAGCAGCGGCAAAGCCCAAAGCTGCAAGGAAGCCGAAAACGGCGGGGAAGCCAA AAGGGAAGACGGTTAAGCCAACACCGGTGAAGAAGGCTGTTGTTAAGACAACGAAGAAGGTTCCTGTGAAGGGTGTGAAGAAGCCT AAGAGCGTTAAAACGCCGGTGAAGAAG

$>$ Contig477

ATGGCTTCTCAATCTCAAACTCAAACTCAACTTAAGCTAACCATTTCAACATCATCACCCAAAAGTGACAGTGGTATAAACGGCGATG TAGCGGCGTCGTTTCTCAAAGGACAGTGCCTATGTTCTCCAACGACACATGAAGGCTCTTTCAGGTGTCGTTTACATCGTTCAGGGGT ATCTTCGTCATCTACACCATCTTGGATGAAACGGACCAAATCATTGCCTCCTAATCATAAAGCTGTTTCTGTTTCTCCACAGTGA $>$ Contig498

ATGTTTAGATCAATGAGTACTCGAAGAGGGTTTGGGACTGGAAGGTATGAGAGATTAGGAAAAGAATCAACAACCACAACACTTTTG AATGAAGAATTCAAGAGAAGCACAAGCTTGCCTTCTAAGGCATCCAATAATTCTTCAAGAAAAGTGGCTCTAGGTTCAACTTTTGGTG ACATAAACCTACAAAGAAACCAAACAAAAAAAGTTAGTAATAATAGTAGCAAGAAGAGTCACCCACTTCTTAGCTTTTTGGATCTTC GTAGGAAAAAGAAAACAACAGCTAAGCCTGAATTTGCAAGGTATCTTGAGTATCTCAAGGAAGGAGGCATGTGGGATTTGAATTCCA ATAAACCAGTTATCTATTACAAGTGA

$>$ Contig522

ATGGAAAAGCAAAACTTGAAATATCGAGGGTGGTGTCCCTCCAACACAAACAATTACGAAATCCACGAAGTAGGAGAGCCTGCACTT CGTAAGCGGCTAGATCGTCCTCGACGTGATAATAAGAAGGGTCATGTGGCATTCAAAACAGTGCTAGAGGAGGCCATAACACCACTA GAAGTGGCAGAGACTGAACGTGTAAATTCTTTTGAGAGCGTGGATCGACAAGCTGATGCGTTTATTAAGCTGGAGCACAGGAGGATT GAACTTGCCAGACTCAAGTCTTTGGGGTTTGCTTGA

$>$ Contig5 52

ATGGGCGAAGCATTGTTCGATCTCGAACAACTTCTCATGTCCAAAAAGGGAAAATTGACACCTCACGAGGTAAATATTCTTCAATCAT GCAAATCAAAAGCTACGAGGGATTTCACTGCTAGTTCACTTGTTGGGGGTGCTGCTGTATGGGCAGCAACTGGGAAACTCCGAACAG CACTTCGAGTAAACCTTTCAGCAGGAGCTGGTGGTTTCCTTGGACTGTGGGTATTCAGTAGATCCTTGTATTCTTCTGCTGATCATATT CTTACTCTGGATGGCAGTATATTACAGAAGGAGTTAGCAAATATACTGGTGACGAAGTACCAGAACAATCCTTCGCTAATGCAGCTTA TATCTAAGCATTTTTATTCGGAGTGGATTTTTGATGATTCAACCTCTAATACTCCTAAATTAAGGTGGCGATACCGTAATTTTTTTAGC GATAATGTAATCAATGGAAGCAGCAGCAGGACTCAGGATTATGGTTCATATGATAAATCGCAAGAAGACACTCAAAATGATTCCTAC GAAAAATCCCAAGGATATTTTGAATCCTATGAAAAATCCCAAGGCAAGTCTGAAAATATCACTGACAGCAAAAGAACAACTCTTCAG ACCAAGAATTTTTTTACAAATCCTGGTCCTGATATCGTGTCAGAGGTAGACCCTCTTGATTGTCTTTTTGGCTATGGTGCTCCAGTGGA GGAGATTTTTCATCCTAATACCCCAAACAAACCATCAGGGACGCATCATCGAGGGCATAGAAGATATCGCCGTAGGCGTCGGATGCG TGATCTTGATGACCTTTCCAACTCAGAGCATGCAGCACCTGTTTGA $>$ Contig549

ATGTATGCTCAATTGTTGCCTCTTGCTTTGCCTGCACCACCAATGCCTGGAATGGGAGGACCAGGTTTGGGAGGAAGCTATGGGCCTC CGCCTCCAGGAATGGGGATGCCCCAAATGCCTCCTTTTGGGATGCCACCCATGGGTAGCAGTTACTAA

$>$ Contig585

ATGTCAGGAATCATCAACAAAATTGGAGAAACTCTTCACATTGGAGGACACAAAAAAGAAGAGGAACACAAAGGTGAAAAACATGA TCAACACAAAGGAGAAAAACATGATGAACACAAAGGTGAAAAAAAAGGAGAACACAAGGAAGGTATAGTTGAGAAAATCAAAGAC AAGATCCATGGTGGTGAGAGTCATGCATGGTCATGA

$>$ Contig596

ATGTCTTCTCATAACATTGCTTTCTTCTTCCTAACCGTTTTACTTGCTACCGGCGCTCTCGGTCAAGCACCTTCTACCTCTCCCACCGCC TCACCCAAATCATCACCGGCTCCGGCGGCATCTACACCTACACCAAAAGCCACTCCTCCTCAAACTCCCACCGCCACTCCTCCTCAAA CTCCCACCGCCACTCCTCCTCCAGTTCCCACCGCTACACCTACTCCCGCTCCAGCCTCACCTCCTCAAGTTTCATCTCCACCTTCTCCAA CACCAACAACAACCACCGTCTCACCTTCCGAATCTCCGGTTGAGTCTCCGATCGATTCTCCTCCCGCTCCCGTCGCTCCTACTATTTCC CCCTCCGCTTCCGAACCTGCTACCGTCGGCGATGGTCCTGCTCTGGCTCCTAGCGGCGAATCCGCTTCCGTTAGAGTCGCCGTCGGTG GATCCGTCGCCGCTATTGTGGCATTTGGTTTGATGATGTAG

$>$ Contig597

ATGGCAGGAATCATTAACAAAATTGGTGAGACCCTTCATATAGGAGGGGATAAGAAAGAAGGTGAACACAAAGGAGAGAGCCATGT TGAACAACAACATGGGTATGGTGGAGAGCACAAAGGAGAGCAACATGGTGTGTATGGAGGAGAGCACAAAGGAGAGCAACATGGTT TGTTTGGTCATGGAGGTGAGCACAAAGGAGAGCAACATGGTGTGTTTGGAGGAGAGCACAAAGGAGAGCAGCATGGTGTGTATGGT GGAGAGCACAAAGGAGAGCAACATGGTCTGTTTGGTCATGGAGGAGAACACAAGCCAGAACAACATCATGGAGAACAAAAGGAAG GATTTTTAGAGAAGATCAAGGACAAGGTCCATGGTGAAGGTGGAGAGGGTGAAAATATCAAAAAGAAGAAGGATAAGAAGAAACGT GGTGAACATGGTGGTGAACATGGCCATGACAGCAGCAGCAGTGATAGTGATTAG 
$>$ Contig618

ATGGCTCAAGAGTACTTTGAACCTGCTAAAGGTGGTCTGATCATCGATGTTAGCTGTGGAAGCGGATTATTTTCCCGAAAGTTTGCCA ATTCTGGAACCTATTCTGGAGTCGTTGCTCTAGATTTTTCTGAAAATATGCTTCGCCAATGCAATGATTTCATTAAGAAAGATGACACA CTTTCATCCACTAATATCGCCCTTGTAAGGGCAGATGTTTCTAGGCTTCCCTTTGCATCAGGTTCAGTTGATGCTGTTCATGCTGGTGCT GCTTTGCATTGCTGGCCATCTCCGTCCAATGCTGTTGCTGAAATCACCCGTGTACTAAGAAGTGGTGGAGTATTTGTCGGAACCACTTT TCTTCGTTATACTTCATCAACTCCGTGGATTTTACGCCCCTTCAGAGAGAGGACTTCTCAAGGCTATGGCTTTTTAAAAGAGGAAGAA ATTAAAGACCTTTGCACCTCATGCGGTCTTACAAATTACTCATGCAAAATCCAGAACTCTTTTATTATGTTTACAGCTCAGAAGCCATA $\mathrm{A}$

$>$ Contig621

ATGGCCGGTTTGCCTTTGAAGTTTCGTATTCAACCCACCGACGTCAAAGCCGCCGCCTTGTGGGGCGTTACCGCCGCCACCGGGGCTC TCTACCTCGTTCAGCCGTGGGGCTTTTTGAAGAGGACCTTCTTCGAGAAGCAAGAGCCTGAGCAAAAATGA

$>$ Contig635

ATGGAAATAAAGAGGTACTTAAGTAGACGTGGCTACCGGCGTCTCGACGGCAGCATATCCACCGTCCGACAGAAGAAGATGCAAGTC ATACAAATGAAAGGAAGTCCTCATAAGCATTGGAGGATTGGAACAAGCCCAAGGCTAAGATGGATGATGAAATCACCATTGAAATTG TTGACAAAAGTTAAGAATATTTATATGAACTTTATGTTTAAAGTGGCTGGGAATGTAGGAGGGGCTTTGAACACTGATAACAACAACC TTGGTGTGAAACAAATTCCACAAGCTCGTCAAAAGTCTAAAGGTTACTACTCTGGTGATGCGTTTGAGGCTAGGCTTATATTTGAGAT TTCCAAGACCTTGGTTGCTTCTCATGAACTATATTCCATTTAA

$>$ Contig655

GATCAAACTTCAGTGGGAAGCTGGAAGCCACAAAGGGAGATCCATGTCCCAGCTAAAG

$>$ Contig655b

AAAATGGGGAAGTAAACGTGTGGGATGAAATCAACGAGGCATTAAAAGCAAAAAAGGCCAAGCTGTCCCTGGACAATGGCTGGGGG AAAAAAAATAGTTCAGGCAATAAGTCATGGTCACAGAAAGCCTTGAGATGCAGTGCACTTGGTCCCACAATGGCTCTTTTAAGGTCC CAGAATGGACTTTAA

$>$ Contig712

TGGAGCTTGACAGGAGTTGGAGCTATATGTTCTTTTGGTGTTGTTGCTGCCACAATTTGTGTCTTGTTCTTTGGAAGCCAACAAAAGAA CAACAAATTACAGCAAGACCAACAAATTAGGTTCAAGATCTACACTGATGACAAGAGGATTAAGCAAGTGGTACAACATGCAACAA AATTGAATGAAGCATTTGCAGCAGTAAGGGGTGTTCCATTGAGCAGAGCTCACATAACTTATGGTGGTTACTATGATAGTGTTTGA $>$ Contig770

ATGGCGTTGGAATGGGTAGTGCTAGGCTACGCCGCGGCTGCAGAAGCAATAATGGTCATCCTCCTCACCATCCCTGGCCTCGACGCTC TCCGTAAGGGTCTAATTGCCGTTACCAAGAACCTTCTCAAACCGTTTCTTTCGGTTGTTCCGTTTTGCCTCTTCCTTTTCATGGACATTT ATTGGAAATACGAAACCAGGCCTAGTTGCGAAGGAGATTCCTGCACTCCCACGGAACACCTTCGTCACCAGAAATCGATTATGAAGA GTCAGCGTAACGCGCTTCTCATCGCCGCCGCGCTTCTTTTTTACTGGCTTCTTTACTCCGTCACCAATCTCGTTGTTAAGATCGATCACC TTAACCAGCGTCTCGAACGTCTCAAGAGGACCGAGTGA $>$ Contig774

ATGACTAAGTTGTGGACTTTGATCACCCAACTTCATTCCCTTGCTGGGCCGGTGTTGACGTTATTGTACCCTTTGTATGCATCGGTGGT AGCGATAGAGAGTCCATCGAAGTTGGATGATGAGCAGTGGCTAGCTTATTGGATAATCTATTCCTTCCTCACCCTTGGGGAAATGCTT CTTCAACCTGCTTTAGAGTGGGTACCAATTTGGTACGATGTGAAACTATTGGTGGCGGCATGGCTTGTGTTGCCGCAGTTTAAAGGAG CTGCTTACTTGTATGAGAGGTTTGTGAGAGAGCATATAAGGAAATATGTGACGGAGCGGGTCCACCACCATCCTCAGAATGAGAACC AGCTGCTGCAACACACCAACAAAAAATCAACCAACGGGGGGGGATCATGA

$>$ Contig834

ATGTCTCTAATTGTTGAGAAAACCAACACCGGCCGTGAATACAAGGTCAAGGACCTTTCCCAGGCCGATTTCGGCCGACTCGAAATCG AGTTGGCCGAAATCGAGATGCCCGGACTCATTTCCAGTCGGACTGAGTTCGGACCATCACAGCCCTTCAAAGGAGCCAGAATCACCG GATCTCTCCACATGACGATCCAAACCGCCGCTCTCATCGAAACCCTAACAGCGTTAGGTGCCGAAGTCCGTTGGTGTTCATGCAATAT TTTCTCAACACAGGACCACGCCGCCGCTGCCATAGCACGTGACAGCGCCGCCGTGTTCGCCTGGAAAGGTGAGACTCTTCAGGAGTA TTGGTGGTGTACTGAACGCGCTTTGGATTGGGGTCCAGGTGGTGGTCCTGATTTGATCGTTGATGATGGTGGTGATGTTACGCTTTTGA TTCATGAAGGTGTTAAGGCTGAAGAGCTTTTTGAGAAAACTGGTGAGGTTCCTGATCCTTCTTCTACTGATAACGCTGAGTTTCAGATC GTGCTTAGTATTATTAGAGAGGGTTTGAAAACTGATCCTCAAAGGTATCGTAAGATGAAGCAAAGGCTTGTTGGTGTTTCTGAAGAGA CAACTACTGGTGTTAAGAGGCTTTATCAGATGCAGGCTAATGGAACTCTTTTGTTCCCTGCTATTAATGTCAACGACTCTGTCACCAAG AGCAAGTTTGACAACTTGTATGGATGCCGTCACTCACTTCCTGATGGTTTGATGAGGGCTACTGATGTTATGATTGCTGGGAAAGTGG GTGTTGTCTGTGGATACGGAGATGTTGGAAAGGGTTGTGCTGTTGCCTTGAAACAAGCTGGAGCACGTGTGATAGTGACTGAAATCG ATCCAATTTGTGCCCTTCAGGCTCTGATGGAAGGTTTCCAGGTTCTGACTCTTGAAGATGTTGTTTCTATTGCTGATATCTTTGTTACCA CCACCGGTAACAAGGACATCATCATGGTTGATGACATGAGGAAAATGAAAAACAATGCCATTGTTTGCAACATTGGTCACTTTGACA ATGAAATTGATATGGCTGGTCTTGAGAACTACCCTGGTGTGAAGCGCATCACCATCAAGCCTCAAACCGACAGATGGGTTTTCCCTGA GACCAACACTGGGATCATTGTCTTGGCCGAGGGTCGATTGATGAACTTGGGTTGTGCTACCGGACACCCTAGTTTTGTGATGTCATGC TCGTTCACCAACCAGGTTATTGCTCAA

$>$ Contig834b

TTGGTGGGCATGGGTTAGAGGAAGGCAACCCATTGGTTTCTGTTGA

$>$ Contig843

ATGAAAATCAGTGAAAAAGAGAGTAAAAAAAGTGGTGATGATGATGATAATGGAGTGAAGTTGGAAGAGAAACAGGGCATAAAAA CAGAGACAAGTTTGGAGTTGAAGGAATATAGGAGGTGTCAATCGGAAATTACGATTGTGGGGGGTGTGAATAGTAATGATAAAAAG GATGAGAGAGCGTTGCAGAGGTGTGAGAGTGAAAAAAGCAGTCTGTGTTCGTACCCTGAAGATGGAATGAGCAGTGACGAATTTCGT CAAACCGTTGAGGCATTTATTGCACGACAACAGAAGCTACGAATACACGAATTTCAAAACTTGTATTCCTAG

$>$ Contig850

ATGGCTGCTGCTCAATTCCTCACTCTTCTTCACTCTTCTTCAAGATCTTCTTTGCTCAAACCCTCTAGACCATTTTTCTACAACCCCATC AAGAATTATGGGGAATCAATCAAAGGAAAACAAAGCAGATTGATGGAAGAAAGGGCACCATCTACAGCTGAAGAATTTCTAAGAGT GGCAGAGGAGAAAGAAAAGGAAACTAAAAAAGTGGTGGCAAGTCAAACTATTGATAAAACAATTGATGCTGCTGAAGAAGCTACTA AGGGTAATTCAAGAATTGAAAATGTTAAGAATAGGTACAAGGAGGGGTGA

$>$ Contig910 
ATGGCGTTCATGAAGGGAAAGGCAGTGATTACCAAAGTGGCAGCTTTAGCGAGTTATGGTGTGTTACCAGGTGCCATGGTGGCTGCTT TCGTCTACTCTCCCCCTAATTACGCCGAAAAACACGCCCCCGCCACCTCCAATTAG

$>$ Contig 1007

ACCTGAGAAAACGGAACGATAAGAGTTCCTTCCAACTAGAGACAGCGTCGGTCTCGAAGAGATCCAGCGCCGATGATTCAGCGACTG GGAAGAGCAGCAGCTCGCCTGTGGAGAAGGTGACGATTTTCCGGAGAGGCCAGTCGCTGGATTCGTCGTTGGTAGCGGCGAAGAATG ACATGTACGCTGGATCTGCGTTCGCGATGTCTCCGTCACCAAGCGCGCTTCCTCTGCCGTCTTTTATGACAAAGAAGCAGTCGCTAGA GACGTTTGACGACTCCGCAACGCGAGATCTGAGGCGTTTGCTCCGAATTGATTGA

$>$ Contig1121

ATGGCTTCCACTAAAGTGATGGTTCTTGCAACAATGTCGGTGCTCGCCGCCGTCCTATCCGTCGCCTCCGCTGCCGAGGCTCCGGCTCC TAGTCCTGTATCACCAGCCACCGCAATTTCGCCATCGTTCATTGTAGGTTTTCTCGCCGCTGTCGCTGGTCTCGCTTTTGGATCTAAACT CCAGATCTGA

$>$ Contig1131

ATGCAAAGAAAGATGCAAGGATACCCACAGCAACAGCAATGCTATGGTAGTGATGATGGCATGTGGATGGAAAGTAAGGGACAAAT GCAGCCACAGGTCCATCACTACCAGGACGATTCTTGGTCTAATGATAACAACAACCAGAAGCAACTATTTCCTGCAGCTATGCACATG AAACCGGCCGCTAATTTTGGAGGAAACAACCACCATATGTATCCTCATCAGGAAACCATGCCTGCTGCTGCAGTTGGTTATGGTAATC AAATGCATGGAAATGGTGGCAAACAATTCCCTTATGGTGGTACTCATAATCATTCTCCAAATGGTGTGAGGCCATTCAATCTTGAAGA GTATGAGTATGAAGCATACAAGGAGGAGCATGTTGGTGGTTCAAAGAAGGATGAAATGAGATATGAGCGACATGGATATGGAAATG GAGGTGATGTTCGCTATGTCAATTCATATGAATATAATAACAACTTCAACGCCCGTATTAAGCCTCATGCTCATGGACCCCACAAAGT CAACTGGACACTAAAGGGTGTCTAA

$>$ Contig1132

ATGGCGAAGAAAGTAGCAGAGAGAGCAGTAACAGCAATGAGGGGTTTACTGAAGGAAAATGCTGCAGACGGTGAAGATGGAGTTTC AGAGCCACCGTCGGGTCCGGGTCCCGGAGAGGGAGTATCAGGTTCAGGAGGAGGGGTAGAAGTGGAAGGAGCAGGTGCTTTGGATG GAGTACAGCACTTGGAGCCTGTGCTAAACAAGATGTGGCTAGTAAACTAA

$>$ Contig 1134

ATGGCTGGTTTACTAAGGAATGTATTGGGTTTGAACTATGGTGGAAACAATGGCACAGTTGATGCATTTAACAACAAATTGGGTACAC AAGATTACGATGAAGCTGAATTCAACACTGGTGCTGAAATTAAAAAAGGAAGTTACCCAACTCATAGCAACAATGGAACCAAAAAG GCATTCAACAACTCATGGGGAGGAACTCAGAAATTCGGAAAAGCTAAGTTTAACACTGGTGCCAAGATTGGAAACTAA

$>$ Contig1156

ATGGCAGGTATTGCATCCGGCATCTGCAACCTTGCTGTTGGCGATAATCGACGACGACCATTGCAACCACAATCTTCTCCTCCTCCTCC TCCTGCGCCGCAACAGTCATCAGAAGAGTTCGCCTATTCAAATAGTGCAAGGCAAAATATAAAAGGTCTTACAAATCAAACTGGCTA TGTCAAGGGAAATGCTAATGGAGTTATCAACTTTGGTACCTTGACATCCTCTGCTGCTCAGAGGCAATCATGA

$>$ Contig1158

ATGGGTGGTGCATTTTGGGGGACACGGGTGATGGAGATAGTGAAAAAGCATGATTCTGGAGGGCTCGTTTGGAAGAGAATCAAGCTT ACCACCACTCGTAAAGCCAATGCCAAGAAACGTCTTCTCCGTGTTTGGCAGAATGAAGCTGTCCTCAAGGCATGTTTTGAACCATCTC CTTCAGCAAGTTCTTCCGGCAGTGCCAGCAAAGCTAGTAGTTAA

$>$ Contig1165

ATGGCGAAAAAAGTAGCAAAGAGAGCAGTAACAGCAATGAGGGGTTTACTGAAGGAAAATGCTGCAAACGGTGAAGATGGAGTTTC AAAGCCACCGTCGGGTCCGGGTCCCGGAGAGGGAGTATCAGGTTCAGGAGGAGGGGTAGAAGTGGAAGGAGCAAGTGCTTTGGATG GAGTAAATGCTGGTGTTGGTGATGGAAGAGAGGTTGCCGGAAAAAAAGCTGGTGCAAGAAAGGTTGGAGAGGGAGTAGGAGGTGCT GTAAGTGGTGGTGTTGCTGGTGGTTGA

$>$ Contig1173

ATGACGGTGACACCTAAAATCTCAGTTAACGATGGAAACTTGGTTGTTCATGGCAAGACCATACTGAAAGGAGTACCAGAAAACATT GTCCTCACTCCAGGTTCTGGCAATGGCCTTCTTACCGGTGCATTTATTGGTGCTACTGCTTCCCATACCAAAAGCCTCCATGTCTTTCCC ATTGGTATCTTAGAGGGTCTCAGGTTCATGTGTTGTTTCCGGTTCAAGCTATGGTGGATGACGCAGAGAATGGGAACTTGTGGGAGGG ATATTCCTCTAGAGACCCAATTCATGCTCATAGAGACCAAACACACCGAAGGTGAACCCCAAGATTCTCCTATCATCTACACTGTCTT GCTTCCTCTATTGGAAGGTCCATTCCGAGCTGTTCTACAAGGCAATGAAAATTGCGAAATCGAGATTTGCCTTGAAAGTGGAGATCAT GCTGTTGAGACTAATCAAGGCCTTCACATGGTTTACATGCATGCTGGCACCAACCCTTTTGAAGTCATCAACCAAGCTGTCAAGGCTG TGGAAAAGCACATGCAAACATTTCATCATC

$>$ Contig1173b

CTGAGGGTGTTGAGGAAGGCTTGAAAAGTTTATCAGAGGGAGGTACACCTCCACGATTCCTCATCATAGACGATGGTTGGCAACAGA TTGAAAGTAAAGCAAAGGATCCCGGCTGTGTTGTGCAAGAAGGAGCACAGTTTGCTACTAGGTTGACTGGGATTAAAGAGAATGCTA AATTTCAAAAGAATAAGAATGGACAGAACGACGAACAAATACCAGGTCTTAAACATCTAGTAGATGGAGTAAAGAAACATCACAAT GTCAAAGATGTTTATGTATGGCATGCACTAGCTGGTTATTGGGGTGGAGTGAAGCCAGCAGCAACTGGCATGGAACATTATGACACC GCCCTGGCGTACCCAGTGCAGTCACCAGGAGTACTTGGAAACCAACCGGACATTGTCATGGACAGCTTGGCTGTGCATGGTCTTGGTC TAGTACATCCAAAGAAGGTTTTCAACTTCTACAATGAACTCCATGCTTACTTAGCATCATGTGGAGTAGATGGAGTGAAG $>$ Contig1173c

TCTCGCTTACACGCAGCTATCATCATGCGCTTGAGGCTTCCATTCGCTCGTAACTTTGCTGACAATGGATGCATTGCGTGTATGTGTCA CAACACTGATGGACTTTACAGTGCTAAGCAGACTGCTATTGTGAGAGCCTCTGATGATTTTTACCCGCATGATCCTGCTTCTCACACCA TCCATATTTCTTCTGTTGCATACAATTCGCTTTTCCTTGGAGAATTTATGCAACCAGATTGGGACATGTTCCATAGTTTGCACCCAGCA GCAGAATATCATGCAGCAGCTCGTGCAATCGGTGGATGTCCAATTTATGTCAGTGATAAGCCAGGCAACCACAATTTTGATCTTCTTA AGAAGCTGGTTCTCGCTGATGGTTCAGTTCTTCGTGCACAGCTACCTGGCAGGCCTACCCGTGACTGTCTCTTTGTTGATCCGGCGAGA GATAGGACTAGCTTGCTCAAAATATGGAACATGAACAAATGTACTGGAGTTGTTGGTGTATTTAACTGCCAAGGTGCTGGGTGGTGCA AGGTAGAGAAGAAAACTCGCATCCACGATACATCTCCTGGTACACTCACTAGCTCTGTCAGTGCCTCTGATGTTGATCAAATCAACCA AGTAGCTGGTGTTGAATGGCATGGGGAGACTATTGTTTATGCTTACAGATCAGGTGAGGTGATACGGCTACCAAAAGGGGTTTCGATT CCAGTGACACTAAAGGTTCTGGAGTTTGAACTTTTCCATTTCTGTCCAATCCAAGAAATAGCACCAAGTATATCATTTGCCGCAATAG GCCTAATGGATATGTTCAACACTGGAGGAGCTGTGGAAGAGGTTGAAATCCATAAAGCCTCCGACAACAAACAAGAACTATTCGACG GCGAGGTTGTATCTGAACTGACAACTTCTCTCAGTCCTAACCGAACGAAAACAGCAACTGTTGCTCTAAAGGTCAGAGGAAGTGGCA AGTTTGGAGTTTACTCTTCTCAGCATCCACTTCAATGTGCAGTCGATGGCATTGATACCGATTTCAACTATGACTCAGAAACCGGGTTG ACAACCTTCTCCATCCCAGTTCCTCAAGAGGGGATGTATAGATGGTCGATAGAGATTCAAATTTGA 
$>$ Contig1204

ATGGATCCAGACGCGTTGGCAAAGGCATTCGTTGAGCACTACTACACTACCTTTGACACCAACCGTTCCGGTCTCGCCAATCTCTATC AAGAAGGTTCTATGCTTTCCTTCGAAGGTCAGAAGATTCAAGGTTCTCAGAATATTGTCGCCAAACTCACCACTCTCCCTTTCCAGCA GTGCCATCACTCCATCACCACCGTTGATTGTCAACCTTCTGGTGTCAACGCTGGCATGCTCGTCTTCGTCAGTGGTAATCTCCAGCTCG CCGGCGAACAACATGCCCTTAAGTTCAGTCAGATGTTTCATTTGATACCAACACCACAAGGAAGCTATTATGTGTTGAATGACATATT CCGTCTGAATTATGCATGA

$>$ Contig1216

CATCATGGTGAACACAAAGGGGAGCAGCCTCATGGGTATGGAGAGCACAAGGGAGAGCACCATGGTGAAGAGCACAAAGAAGGGTT CATAGACAAGATCAAGGACAAGATCCATGGTGAGGGAGAGGGTGGATGA

$>$ Contig1224

ATGGCTGCACTTTGGTCATGTTGTTTACTTGCACTGCTTGTTTTGATGTCAGCTATTGTAACTGAAAGTAGAGTTGCAAGGAAGGACTT AGGTTTGGATCTTGGTGGTTTGGGGATTGGACTTGGAGCTGGACTAGGTTTGGGTATTGGAGGTGGTAGTGGCTCTGGAGCTGGAGCT GGAGCAGGTTCTGGTTCTGGTTCAGGTTCTAGTTCTTCCTCGAGTTCATCGTCTTCGTCTTCTAGTTCAGGGTCTGGTTCTGGTGCAGGC TCTGAAGCAGGCTCGTATGCTGGATCTCGAGCTGGATCGGGATCAGGAGGTAGAAGTCGCATTAAAGAAATAATGCATGAATGACTT GAGTTGTGGAATGAGAGGAATTCCGGCAAGTATGAAAAGAAGGTTTACGTTTTGCCCAAGCACCTTGATGAGAAGGTGGCTGCACTT CATCTTAACAAGCTTGGTGCTAAGCTCACCAAGCTTTCCCAATCCCAGGCTGATTACATCAGTGTTCCAGTTGAGGGTCCATACAAAC CTGCTCACTACAG

$>$ Contig1227

ATGGCATCAATGACCGCATTCAAGGCTTTCTTCTTTGCCGTTGCTCTACTGTCCGTCGCCGTTTCTGCTCAAGAGACGTCTCCTGCGTC GTCGCCTGCACCGTCTCCTGACGCCGGAGCAGCAGGATCTATGTCCACCTCAGTTGCCATGATCGGCGCCTCGCTCGTGTTGTCCATG CTTGCCGCGTTCAAGTTTTGA

$>$ Contig 1235

ATGGCAAACATCGACATCATAATTGAAGCTCCAATAATAGCCACTGAACCTGTAACAGATCCGGCAGCTCCTGCATCGGGACCCGGT GCCGGAGCGGGGGAAAGACCGAGGTCTTGGGCGGAGGCCACCGCGGCGAAAACAAATACAAAAAAGAAGGAAAATAAATTGAAAG TCGTACCAGATGATGCCATTGTTAAAGTATAA

$>$ Contig 1258

CACCACCAGCACCACCAGCAACTGCAGAGGTGGAGGAGGAGAAAGAGAACAAACCAGTTGAGCCAGTGGAAGCTCCACCAGCAGCA GTAGCAGAAGTTCCAATTGAGACTCCTGAAGGTTAG

$>$ Contig1262

ATGGAGAACAACGGCAGCCTTTTATCAAGGGAGAAGATCGATCAGATGGCTACCTGGGTGGGGTCCACCGTCTCTTCCGCTTTCTTCT CTTCCTTGGAACGCTTTTCTTGTGTTAACGTCGCCACTTCTGACCCTGACAACGACGAGGACGACGACGATTACTCCGTCACAACAAC CACAACAACCGCCACCACCACCGCCGACAGCAACAATCCTAATCCTGACGTTCAGGTCAACGGTCATAATACAAACGACGTCACCAA TCTCCCCGTTTGA

$>$ Contig 1266

ATGGCCACTTCTTTCGCACCATTATCCATTTCGGCAGGTGGATCTCATCTGAAGTCACCGGAGCTGTTTTTAACAAAGCGTAATTCATC TGTGGTTGGATCTAAGCTTGCTGTTCAAAGGAAATCAAACCTTGTAACCAGGAGAAGGAACCTTACTTCACCTGTTCGCGCCGAATAT AGTGATAGCAGAGGAGGTGGAGGTGGGGATTTTGTTGCTGGATTTCTTCTGGGTGGTGCTGTTTTCGGAACTCTGGCCTATGTTTTTGC TCCCCAGATCAGAAGATCTCTCCTAAATGAAAATGAATATGGGTTTCGGAAGGCCAGAAGACCAATCTATTACGACGAAGGGCTAGA GGGGACCAGGCAGACCTTGAACGAAAAGATAGGGCAACTAAATTCTGCCATTGATAATATATCTTCACGGCTGAGAGGTAAGAATAA TGTGCCTGCTGCCCCTATAGAAAGTGATCCTGAAGTTGAAGCTACCATGTGA

$>$ Contig1320

CCTATGAGGTCAAAGCCACACCACAAATTGAAGGTTTTAGCACTTCAAAGCAACAAAGGAAGCCTAAGTCCATGTATGGTATGACGT TGGAATCAAGCAAACCATTACCAAACACTCAAAGAACAAATTTAGGATTGCATGTTGTTCAACATACAACGATGTCAGAGAAAACAA AGACTGTTTATGGGGGAGCTAATAATGTGAAGGGTCCACATAAGGGAAAAAGTAGTGCAAGCACCAATTCAATCAAATTTTCATCAT TGTTCATGGCTGCACTAAGCCATTTCATTATAGCAACGATTATAGTTGGTTGCTTTTTTTGA

$>$ Contig1338

ATGGAGGATTCCACCGACAAGAGAAAGCGAGCTAGAGACGAGTCAGGAGACTCACCTGACTTGCCCGATTCCGAAACTCACCGAGTT GACTCACATGAGACTAAGATTCGACGTGTTAACTCATGTTCTGATGTTAATTCGTCAGAGTGTCAGCTTACCCGAGTTGACTCGGCCG AATCGTGTCTTAATTCGGTTTTTGACTCCGGTGTTCAACTTCAAGATGACATTTTTCATATACTTGATGATACAGATAATGTTCCTGAG CGTGAATCAGTGATGGGTCTTGACTCAGTGATGAAAAGCTTCGAAGATGAGATTTTAGCTCCGGGTTTGAATTCGGGTCAAGTTGACC CGACTCAGACGACCGGTTCCGGAGAGATGGAGATGAATTTGGGTTACTTGTTTGAAGCTTCCGATGATGAACTTGGGTTGCCACCGAC GGTGGGTAATGGTGATGAACCGGGTCGAGTTGAACCGGAAATTGTGGACCTGACCGGGTTCGTGGGGTTTGAGGATGATTTTAACGG TTATGATGAGTTTGGTTTTGGAACAGGGTTGTTGTCGGAGTGTGACGGTGATAACGGTGGTGCCGGAGGTTTTGTAACGGTGGATGGG TTGTTTGAATATGCGGAACCGGCGGCGGATGTTTTGTGGCGGTCGGAGTCGTTACAAGCTATGTAA

$>$ Contig1360

GTCACGGTCCCAGTTGGGACTACAGTTGGGGTTGGGGCTCCTCGCCCGGAAGTGGATACGGTTACGGTTCAGGTTCAGGGCATTTTCC TTTTGGTGTTGGTAAAGGTTTTGGAGTTGGTTTTGGAACCGGTACTGGATTTGGATCCGGGTATGGTTATGGATCGGGGGGGGGTGGT GCTCATGGTGGTGGTTTTGGAAGTGGTTCTGGCGGCGGAGGTAAAACCAACCACGGATAA

$>$ Contig 1362

ATGTCTAAGCCCTCCTTCAAGATTCAACATCCTCTGGAAAGAAGACAGGCTGAAGCTTCTCGCATTAGAGAGAAGTATCCCGATAGA GTACCTGTGATTGTGGAGAAAGCTGGAAGAAGTGACATTGCAGACATTGACAAGAAGAAGTACCTTGTCCCAGCTGACTTGTCTGTT GGTCAATTTGTCTATGTTGTCCGCAAAAGAATTAAGCTCAGTGCAGAGAAGGCTATCTTTGTTTTCATCAATAACACTCTACCTCCAAC TGCTGCTCTGATGTCTGCTCTTTATGAGGAACATAAGGATGAGGATGGCTTTCTTTACATGACTTACAGTGGAGAGAACACCTTTGGA TCTCACTAG

$>$ Contig 1367

ATGGCAAACATGGACATCATAATTGAAGCTCCAATAATAGCCACTGAACCTGTAACAGATCCGGCAGCTCCTGCATCGGGACCCGGT GCCGGAGCGGGGGAGAGACCGAGGTCTTGGGCGGAGGCCACCGCGGCGAAAACAAATACAAAAAAGAAGGAAAATAAATTGAAAG TCGTACCAGATGATGCCATTGTTAAAGTATAA $>$ Contig 1367 
bGTTGGAGAGATTTGCGAAATGATATCGACGACACTAATAGGCGTGATATTTAGGAAGTGGTGTTTAGGAAGATATGATCAGGTA $>$ Contig1371

ATGGCTGATGACAACAACAGAGGCTCCTCTGCTTTGGATGGTTTCACTCTGAATCCTCTGCCTTATCCTGTTCTGTTAATCTTAGCAGT GATCTTCATCTTCCTTGGTACTTCATGGTACTTTTCTTATGAAGAAGTTGTTGAAACTGCTCAAGAACAATTGGGTTGGGTTTTATTTGT TGTACCAATCGTGCTTATACTTATAGTTCGTTTGGTTTCATCAATGGAAGATTCAGGTTGGTTTTTTGGTGGTTCTGGTTGGGAAAGGC GCAGGACAACACAAGAAAGCACCTCTGGAGGGAGTTCTCCTTGGGGTGTGGCTGCTTTGATTGTTGTTTTGTTGATATTGGTGCAGTT TCAATCTGTGTTTCTTGATAGTTGGTTTTATTGA

$>$ Contig1396

ATGCTACCATTGAACAACATCATCTTTCTTATCTTTCTCATCATCACTTCCACTGCGGCAACAGGAAGAAAGCTAAGTTTCGATATGTC AAGCGGCAGTGCACCGACGTCAAGTGGTGGTAGCGGGACAGGTCACGGTCCCAGTTGGGACTACAGTTGGGGTTGGGGCTCCTCGCC CGGAAGTGGATACGGTTACGGTTCAGGTTCAGGGCATTCTCCTTCTGGTGTTGGTAAAGGTTTTGGAGTTGGTTTTGGAACCGGTACT GGATCTGGATCCGGGTATGGTTATGGATCGAAAACCAACCACGGATAA

$>$ Contig1402

GATAGTGGCCTACCACGGTGGTGGTGGTAATGGAGGTTATGGCAGTGGCTCTGGGTATGGTTCGGGATATGGTTCCGGTTCGGGAGG AAATGAGTACCCTTAA

$>$ Contig1406

ATGGCAACCCACAAGAAGGAGGTAAAGATGACCCAAGCAGAGCTGGACAAGCAGACGGCGCGTGAACATAACGCCGCTGTTAACCA GTCGACAACGGAAGGGCAAATGGGACATACTGCTGGGGCCCATCCGATGTCGGCATTGCCTGGTCATGGAACTGGACATCCCATGGG GCATCCCACGGAGGGAGTGGTGGGCTCCCACCCGATTGGAATTAATAGAGGCCCAGATGGGACCGTTACGGCCCATAATTTTCGTGTT GGTGGAAATCCGAATTCCCCAGGGTATACAACTGGCGGTTCTTATACTTAA

$>$ Contig1431

CTAATGCTGCTACACATGGAGGAGGACATGGTTATCCACCTGGGGCATACCCTCCACAACAAGGATACCCTCCACAACAAGGTTATC CACCATCTGGGTATCCTCCACAACAAGGATACCCTCCACAAGGCTACCCACCTGCTGGTTATCCTGCTTCATCTGGTCATAATGCTCA AGGGTCTCATGGACATGGCGGTCCCGGTATGGGAGCAATGCTTGCCGGGGGTGCAGCTGCCGCCGCCGCTGCTTATGGTGCTCACCAT ATCTCCCACGGCTCTCAAGGTCACTATCCACAAGGTGGATACGCACCAGGTGGCTATGCACAAGGTGGATATGCACATGGTGGTGGT CACATGCCTCACGGAAAATTCAAGCAGCATGGCAAGTTCAAGCAGGGAAAGCATGGCAAGTTCAAGCATGGAAAGTTTGGCAAGCA TGGTGGAGGCAAGCATGGGTTCAAGAAGTGGAAGTGA

$>$ Contig1471

ATGGAGGCATTGAAGATGAATGTTTTCGTAGTAGTTATGGTTGCTATGTTGGTAATGGTAGCTAGTGGTGTTTCAGCTGCTGATGCAC CAGCTCCAAGTCCTGTTTCTGATGCAACTACACTATTTGTTCCAACTGCTTTTGCTTCTCTCATTGCTCTTGCATTTGGCTTTCTCTTTTG A

$>$ Contig1476

ATGGCATCATCAACTACTACAATTAAATTATTATCTTTCTTTTATGTCATCGTCTTTGCCGCCGTAGCCTCAGCACAAGACCTTAGTCCT TCGTCGGCTCCGGCACCAAGTCCTGATGCGGGAGCTGCCGGATCCATTACAAATCCAGTGGCTATGATTGGAGCCTCGATCGTGTTGT CTATGTTCGCCATCTTGAAGAACTGA

$>$ Contig 1478

ATGGCCGCCAACAGATGGATTAGACCCGAGGTATTCCCACTTTTTGCCTCAGTTGGTGTTGCTGTTGGGATCTGCGGTATGCAACTTGT AAGGAATATAACCGGCAACCCTGACGTCAGGGTAACCAAGCAGAACAGAGCTGCAGGAGTTCTTGACAATTTTTCTGAGGGTGAGAA ATATTCACAACATACCCTAAGGAAGTTTGTCCGCAACAAGAGCTCTCAGATTATGCCATCCGTTAACAACTTTTTCTCTGATCCAAAAT $\mathrm{AG}$

$>$ Contig 1480

GTGGGGTAGGAGATGGTGGAGAAGGTGTGGTTCCAGATGGTGGAGGAGAAGGTGTGGTTCCAGATGGTGGAGGTGGTGGTGATTAA $>$ Contig1489

ATGGAAAACCAATCCACAACAACCCCATCCACGATGCAGTTTCTCATCCTCGAACAAGTTCAGTTCTTGAAAGTAAACGACGATTCTC TTCTTCAATGGGAACTCGTCGATGTCGTTGACGCCGAAGAAGAATTAGAACAACAATCAATCGATGATGATGGGGATTCTTTCCTTTC TCGTGACCCAATTGAAGGTATTCGACATCGCGTTCTTCATCTCGATGCACATGTAGAAGATCATCACCATCACCGTGATGCGGATGCA GATGGGTTTAGTGAAGAACAGGTTAACCGCTTGTTCCATGGGATATTGGGAACAAGTTAG

$>$ Contig1539

ATGAATACAACGACCAAGACCACCGAAGTATCCACAACTATATCGATCAATATATACAGATTTCTCCCTGAAAACATATGGTCCAAC AAGATCGTCGCTTCGGCAACAG

$>$ Contig $1539 \mathrm{~b}$

ACAAGGCGGCGGAAACACTTAGGAATGACATTGAATGGTTACGGATAACGACGGGTGTGAGCATGGAGAAAGTTGCTTATGAATAA $>$ Contig1546

ATGTCGACCAAGCAAGGTGGAAAAGCTAAGCCTTTGAAGAAACCAAAGTCTGATAAAAAGGATTACGATGAGATTGACATGGCTAA CATCCAGAAGAAAAAGGATGAGGAGAAGGCTCTAAAGGAGTTAAAAGCTAAGGCACAACAGAAGGGATCTTTTGGAGGATCTGGGC TCAAGAAAAGTGGAAAGAAATAG

$>$ Contig1566

ATGGCCTTTGTTCATAGTTTCCAGGTGGAACGCCTAACTTTTGTCCCTCTGGATAAACTCTTCAGAGATGTTAAAAGAAATGCTTTTCA TGTCATCCCAAAGCTGGATCAGATCATCTTGAGAGTTCAAACCCTTCAAGGAGATGGAAGCCCTGGTACCCTCCTCAACGTAATTAAG AAACCTCAAGAGGGTATAATCTCGAAGAATGTGGTGCTCAAAGTTGATGAAATCGACGAGGCTGAGAAGAAATATGGATACAGCGT AGTTGGGGGGACAGATTTCGACGAAACCATGACGGAGAGGATTTCATACAAAATAAAGTTGGAGGAGAGTGATGATTTTGATGGCTG CATCGCAAATATCTCAATCGAATTCCACGGCAAAGAGCGTAGAAATATGTCTGATGAACAAGTTGTTGAGCAAACAGATGTCCAGGG CTTTTACCTTTTCGATGACATTATTGAAGTCACTCAAGAAGCCTGA $>$ Contig 1567

ATGGCAATGAGATCGGCGCTGAGTCGAGGGATCGTGACTCGTTCGATGGACTCAACATCTCGTGGAGCTTTCAGTCGTTTCTACAGCG GCAAAGGTAAGATTCTTAGCGAAGAGGAACAAGCCAAAGAAAACGTTTACATCCAGAAATGGGAGCGGGAAAGGTTGGAGAAACTG AAGCTACAGAATGATAAGGCTAAGGCTGAGAAGGACAAAGACACTACTGATAAGAAACCTGATGGGACTCAAAAGAGCTGA $>$ Contig1568 
TATTTACCCCCGAGGTTCTTGATCTCACTGAGGATGCTCTCATTGAGAAGTTTGCTATTGGTGTCTCAATGGTGACTTCACTTTCGTTG GCCATCTCATACCCAACACTTGCTGCTGCTCCTCACATGTTTGTGAATGCCTACAAGAATGTTCTTGCTGTCGCTGTTGCAACAGAATA TTCTTTCCCTGAGGCCGACACGGTCAAGGAGTTCCTAAAGGACCCAAGCAAGTTCAAGGAGGAGGAGAAGAAGGATGAGCCCGCTG AAGAATCTGATGATGACATGGGTTTCAGTTTGTTTGATTAA

$>$ Contig 1580

ATGTCTTCAACGAAAAAGATCACCCTTAAGAGTTCCGATGGAGAGGCCTTTGAGGTCGATGAAGCGGTGGCTTTGGAGTCACAGACG ATTAAGCACATGATTGAAGATGATTGTGCCGACAGTGGAATTCCTCTTCCTAACGTGACAAGCAAGATCTTGGCGAAGGTAATCGAGT ATTGTAAGAAACATGTTGAGGCTGCGAGTTCTGATGATAAACCTACTGAGGATGATCTTAAGGCTTGGGATGCTGATTTTGTTAAGGT TGACCAGGCTACGCTCTTCGATCTCATACTGGCTGCAAACTACTTGAACATCAAGAGCCTTCTTGATCTTACCTGCCAGACAGTTGCA GACATGATTAAGGGGAAGACACCTGAAGAAATTCGCAAGACTTTTAACATCAAGAATGACTTCACACCTGAGGAAGAGGAGGAAGT TCGTAGGGAGAATCAATGGGCATTTGAATGA

$>$ Contig1582

ATGAAGAGCAAGGGACAGAGCCAGAACACGTTCGTGAGAATCATGTGTTCACCAATAAGAGCACTTGGAAAAGCAAGAGATGCTTA CGTAAGAAGCATAACAAACTGTGGTCAAAATATGCATTATGGAAATCCAATGGATGCTGCTGGAAAATTCTCTGCTTTGTCAAGAAG CCAAAGTGCTGCAACATCAAGAAGATCATCAACAACATCATCATTTGGAGATAATAGTGAAGATTTTGCTGAACTTGTTAGAGCTGCT TCAGCTAGAACTTTGGTTAATAGAATTGACATGGATTTGGTTCTAAAACAACAACAATTGAAGAAATCAAATAAAGGGTTGCCTAAG TCAAGTAGTGTTGGAATGGCTAAGATTGATGAAGATATGCCTTTTGATTATTCTAGTGTTGAGAAAAGGGGTGGTGTTGGGATTGTTC ATGATTCTTATCCTAGATCTAAAAGTTATGCTCTTGGTAATAGGAATGTTGATTTTTGA

$>$ Contig1602

ATGGCGGGAGGCCATAATTACAGTATAAACGTCGACGAAATTAAGGCAGAGGTGAAAAAGATGCTTCATGATGGGCAAGAAGTAGT AATTATTGGGGGTACTCATGCACTACATCACCATGAGAAGCTGACAATTGCGGTTTCAAAAGCTATGCGTGGACATTCTCTTCAGGAA ACCAAGAACGATGGTCGTTTCCATGTTCATACCAAGACTTATCTGGATGGTGCTATTTTAAAAGAAGAGATGGAACGTTCTGCTGATG TTCTTGCTGCTGGATTGCTTGAAGTTGCTGACCCATCTCTTTCAAGTAAATATTTCCTCCGCCAGAATTGGATGGATGAATCAGAAGGG TCAACTGATTCAATTCTTAAGCATAAGCCTCTTTGGTCTTCATATAACTCAAAACATGGCAAGAAAAGGAGAAAGAATGTAAAGAAA CAAGGGGGTCTCCAACCAACTTATGGAACAAGAGTAGTTCCTGTTTTTGTGCTATCATTGGCTGACGTGGACCCAAATCTTATGATGG AGGATGAAAGTATGGTGTGGACAAGCAATGACGTTGTAATTGTGCTTGAACATCAAAATGATAAGATTCCTTTGAGTTATGTTTCAGA AACATATAGAAGGCATGCG

$>$ Contig 1610

ATGGTACAGAAAGTTGTTAGCATGATGCCAGCTCACTTTCAAGTCAATTACAAAGAAACAAGTAACAACGACAAGAACCTCTTGTTA TGCAGTTCTGAGATATTCTACGTACCTCAGCGCTTCGTCAGTGATTTTGTTGAGCTTGTTAACTTAGTAGACAATCTAGAGATCCATCA GAAGGTTGCAATTCCTATGTTCTTTGTCTCCATGGATTCTCCACAGAATTTTGATCCTATTCTTGATACAACAATCTACAAGAAAAAAC CTCCAACCACTAATTCCTCGACTCTTTATTCAGCTAAAGTTCCTGCTGTTCATCCATGGAGTGTATCAACTGAGCAAGAATTTATAAAG CTTATCAGAGTAAGGGCTGAAGGGGATCCACTCCTTATGGAATTGGTTTAA $>$ Contig1627

GGTAAAACTAACCGAATTCTGCTTCGCAATGATAGGAAGAGCCGACATCGAAGGATCAAAAAGCAACGTCGCTATGCACGCTTGGCT GCCACAAGCCAGTTATCCCTGTGGTAACTTTTCTGACACCTCTAGCTTGAAACTTCCAATTAGGCTAAAGGATCGATAG

$>$ Contig1627b

ATGGATTCTGGCTTAGAGGCGTTCAGCCATAATCCTACAGATGGTAGCTTCGCATCACTAGCTTTTCAGCTAAATGCATTTACCAATTA TCTGAATCAACG

\section{Drought related genes}

\section{$>$ Contig48}

GTGGAGGTTATAATCGTGGCGGTGGAGGAGGTGGATACGGAAGCACGTGACCGTGGTTATGGAGGAGGCGGAGACGGTGGTTCCCG CTACTCAAGAGGAGGCGGCGGCGGCGGTGGATTTGATGGAGGAAACTGGAGGAATTAA

$>$ Contig60

ATGAAGAAGAGTTCTAAGGTGGCTGATGAGAACATGTTTGAAGCAGGGAACGAGGTGGAAGTAGCAATATTTGGGGGTGAAAGAGG AAGAAGACAACATGGATGGAGTGGTTTTTCAATTATTTGTGCTATTCTACAAGCTCCAATATCAATACTCTCATGTGTCTCAAATCCTC GTGTTAATGGGCCTGATGGGGTTTGGGCCAATGGTGAATTTGCACAAATTTCTGAGATCAATCATATTATG

$>$ Contig96

ATGGGGTCCCTAATGACCTCTGCTTCTCTCACTCTTGCATTGGCCATTCTCTTTTTAAAGTTACCATCTGAAATTTCAGCAAACCGCCA CGATTCTTATTCATCTCCAACACCACTACTGCCTTTCTACTCGCCACCACCGTCACCAGTCTACTCACCACCATGA

$>$ Contig105

CCATATAAGTATCCATCTCCACCTCCCCCCGTTTACAAATACAAATCTCCACCACCACCGGTGTACAAATCTCCACCTCCTCCCGTTTA CAAATACAAGTCACCACCACCCCCAGTGTACAAATACAAATCTCCACCTCCCCCCGTTTACAAATACAAGTCACCACCACCCCCAGTG TACAAGTACAAATCTCCACCACCACCAGTGTATAAATCTCCACCACCACCCTACAAGCATATATCTCCACCACCAACGCCCAAGAAAC CATACAAGTACGTTTACAAGTATAAGTCCCCTCCTCCACCTGTTTATTCACCACCTCCACCACATTATGTTTATGCTTCACCTCCTCCTC CGGTCTATTCCCCTCCTCCACCGCATTACATCTATGCATCACCTCCTCCTCCTTACCATTAA

$>$ Contig109

ATGAGTAATTACCAGAGACCACCTCAAGAACCTTACCCTCCACCAGGGTTTGGATCTCCTTACCTTCCACCATCACCCCATCTGCATC ACGAAGGCTACCCTCCACCACCACCACCGCATCACCACCGTCCGCCGCACGAATCCTACCAAGGTTATTTCGACAACGGTCATCCACC ACCGCCTCCTCCTCCGGCTCCGTCTCATTACCACTACCAACATATTGATCATCACCGTCATGATGATCACGGTTGTTCTTCCTTCTTCCG TGGCTGCTTGGCGGCGCTTTGTTGCTGTTGCGTGGTAGAGGAGTGTTGTTGCTGTTTCTGA

$>$ Contig 125

ATGGCGAGCTATGTATGGAGAAAATATGCAGATTATTTGTACCCAAAGTGGGAGAAAACATTTCTTTGGGACATGGTCGAACCTTAC AGAAGACCTAAATCCTTCACTCCTGTTGTTGTTACTTATGTCGCTGCTTTCTACCCCGGAGTCGTTGGTGCTGCCATCACTGAACAACT CTACCAGGGAAAATACTGGGAAGAGCATCCTGGGAAAGCTGTTCCTCTCATGAAACCAATGTTTTATGGGGGTCCGTGGAGGGTAAT GGGGGGTCAGGTTCCCAAATATGAGTGA

$>$ Contig141

ATGTCCGCCATGGCAGTAGCCAGAATCGACTTCAATTCTTACAGCAACCAAATTCAGGTTGAATCAAATGGCCACAATGGCTCCAAA ACAGAAACTCACCACTATGGTCAGATTCAGACCCAACATGACAAGAAGCACGGTGTCACCAAAACCCATATCACCGTTTGTGTGGTG 
CAAGCCGAAATCACTCAAACCAAAGAGAACTCTTCTCCTTATGGTGCAAGTGCAACTACAACATGCTTTGGGACTCAAGCTAAGAAG AATAGAGAGATTAACAACAGTAAGAAGGAAACGAATTTGTTCAGGAAGATTAAGAATGGAATGTCCCGCCATAAAGGTGAGGGAAA CAGCAGCAGCAGTGACAGCGAAAGTGACAACGAGAAGAAGTGTCCAAAGACAAAGCATTGA

$>$ Contig144

ATGTATCACAAAATTCAAGTTGTTGATCAGAAAAAAATGAGAATCAAAGTGGAGAATAATATAAAGGGTCGTAGAATTAATGGTTGC ATGATGAAAATCAGTGAAAAAGAGAGTAAAAAAAGTGGTGATGATGATGATAATGGAGTGAAGTTGGAAGAGAAACAGGGCATAA AAACAGAGACAAGTTTGGAGTTGAAGGAATATAGGAGGTGTCAATCGGAAATTACGATTGTGGGGGGTGTGAATAGTAATGATAAA AAGGATGAGAGAGCGTTGCAGAGGTGTGAGAGTGAAAAAAGCAGTCTGTGTTCGTACCCTGAAGATGGAATGAGCAGTGACGAATT TCGTCAAACCGTTGAGGCATTTATTGCACGACAACAGAAGCTACGAATACACGAATTTCAAAACTTGTATTCCTAG

$>$ Contig188

ATGAAGAAGAAGAAAGAGGAAGAGCATGGAGTGAATAGTGGGGAGAGTGATCAGAGTGGTGGAACAGGTGTAATGGTGATAACTA GACCTAAAGGTGGGAATAGAAGTTTGTGTATGGATTTGGAAGAAGTTAAAGCTTGTAGAGATCTTGGATTTGAGTTGGAACATGAGA GAATTTCTGCAGTTTCTTTTTCTAATTCAACAATTGATACTAGTAGGGGTGGTAATTCGCCTATCGCCAATTGGCGTATCTCTGGTCCC GGGGATGATCCAAGAGATGTGAAGGCAAGACTGAAAGTTTGGGCACAGGCGGTAGCAATAGCATCTGCCTCCAAATACGGCTCATGA $>$ Contig200

ATGTTAGCTTCTTCGCTCTCATTCCTTCATTCTTGTGCATTCCCCTCTTCTTCTTTAACAATTCCAACGCCAAATCCCAATTCCAATTCAT CTTTATCTTTTGTCGTTGAAGCCAAATCCACTACCCGAAGAGAAGACCGAACTGCCCGCCATGTCCGTATCAGGAAGAAGGTTGAAGG AACGCCCGAAAGACCAAGATTGTCTGTCTTCCGATCCAACAAGCATCTCTGTGTCCAGGTGATTGATGACACCAAGATGCACACACTT GCATCAGCTTCAACAATGCAGAAGACAATTGCTGAAGAGTTGAACTATACCTCTGGCCCTACAATTGAAGTAGCAAAGAAGGTGGGT GAGATCATTGCAAAATCTTGTCTAGAGAAAGGGATCACAAAGGTAGCCTTTGACCGAGGTGGTTACCCATACCATGGCCGTGTTAAA GCAATTGCTGATGCGGCTCGCGAAAATGGCCTGGACTTCTAA

$>$ Contig221

AGTCATGATGCGTCTTTTGAGATTAAGAAAGGTGAGATGATCTTTGGATATCAGCCGTTCGCTACTAAGGATCATAAGATATTTGAGA ATCCCGAGGAGTTTTTGGCGGAGAGGTTTGTTGGGAGTGATGGAGAAAATCTGTTGAAACACGTGTTCTGGTCCAATGGACGTGAGA CTGATGAGCCTACAGCTGATAATAAAATGTGTCCCGCTAAGAATCTGGTG

$>$ Contig221 b

TGGTATTTTCCGATGGGAATGCACTATAGGGTCGCTGGTTGTGCTTAA

$>$ Contig228

ATAAAATACCAAATAGTGAAGAAGAAGGAATTTTCGAGTCTCCAAAATCTCCATGTGAATCATCCCCTAAACTAAAGAACGCAGGAG TGAACCAGCGATTGGATCCAAACATTCAGCAAAAGCGTAAGCAAGGGACAAAAGCTTTAGAAGATGTGAGTTGTGCTGGTCTAGATG GAACTCCATGGCCAGAGGAGGAGAGAATCAAAGAAGAAGATCTGCGAGGGGATAATCAAGATTACTATAGACATCGTAAAGCGTCT CCATTGTCCGAGTTGGAGTTTGCAGATACAAGAAAGCCTGTGTCACGTGTGATGCACGGGACTGCGGATTCTGGAAGAGGTGGTGAT GTGATTGGCTGGTTGCCGGAACAGCTTGAAGCGGCGGAGGAGACTCTCTTGAGAGCAGCTGAGATGTGGAGGCAGCAGGCTATGCGT GGTGACCCTGATGCACCTCATTCAAGGGTTCTTAGGGCTCTTCGTGGCGAAGACTTTTGA

$>$ Contig261

ATGGATACAAAAAATGAAAATGAAACCACTCCTTCACGTTCACCCTTATTAAACACAAACACAGAAACCACAACACCAACACAAACA CCACAACCAACAACAACAAGTACCACACAAAAAAGTGGCACAGATAGCATTGGAAGCCTCATAAAACTACTCCCAACAGGGACAGT TTTCTTGTTCCAATTCCTAAACCCCGTTGTTACAAACAGTGGTCACTGCAAAACCAGCAACAAGTACCTAAGTTCAATTCTTCTTGCAA TATGCGGTTTCAATTGCATCTTTTCCACTTTCACAGATAGCTACACAGGTACTGATAACAAAAGACACTATGGACTTGTAACACCCAA AGGATTATGGCCTTCAGAGGCTTCGAATTCAATCGATTTATCGAAGTATAAGCTTAAAGTTAGTGACTTTGTTCATGCTGTTCTATCAT GGCTTATTTTTGCAGTGTTGGGTCTTCTTGATACAAACACTGTGCTATGTTTTTACCCTTCTTTTATGAAAACTCAGAAAACTGTTCTTC AAGTGTTGCCTGTAGCTATTGGAGTTGTTGTTGGATGGGTGTTTTCGGTTTTTCCACAACAAAGACATGGAATTGGTTACCCTCCTAGT ACCTGA

$>$ Contig298

ATGAGTCGAAAAAACGGAAGTGGACCAAAGCTTGACTTGAAACTGAACCTATCACCACCAAGAGTTGACCGGAGAATGGAGTCATC ACCGACACGATCAGCGTCGGTGTCGCCGCCAAGTTCATGTGTTTCATCGGAGAATGATCCAAACAGTCCTGAACCAACTTCCATGGTA CTTGTTGGTTGTCCTCGTTGTCTCATGTATGTCATGCTCTCTGAGAATGATCCAAAATGTCCAAAATGCCACAGCACCGTTTTGCTCGA TTTTCTCAAACTCGACAACAACAACAACAATAACAAGAAGAGTTAG $>$ Contig306

GGGAGAGGGATTTTGTGTGTCCTCATGAAGGCTGTGGTAAGGCCTTCTCCTTGGACTTCAACCTAAGGTCTCACATGAAGACACATTC ACAAGAGAACTATCATATATGCGCGTGGCGGGATTGTGGGAAGAGATACGCTCATGAATACAAGCTAAAGAATCACATTGCTTCTCA TCATGAAAAGAGTACACCAATGGAGATGACAAAGTATACCACTCCTCCATCAGAAAAGCAAACAAAAACTGCTAAACCTTCTGGGGG AGCATACGGTTCGGCATCCTCTGATCGCCCATACGCATGTCCCCTATGA $>$ Contig334

ATGCCTGGATGGGAGCCCTTAAGCACTATAAACAAGCCCCCCGCCGCGGGAGCGATACCCCTGCAATCCGAAGATACGAGAAGTGGT GGTGGATTCATATATAGCACTACGTACGCTGTCAACGAAGGCTTCCCCGGGGATGGATATGACCCCGGGCCCCCTGTATGGTATGGAT TCAGCTAG

$>$ Contig350

ACTCTTCTCTTTTCCATCAAAGCTAACTCAAGTGGTGTATTGCTACTTGATGATTTTGAGATAACACATTTCAACAGACCAAAGGACAA GTTGTTGGATTGGTTCTTCAATCCTGTTATGGTTCTCAAGGAACAAATAAGGGTCATTAAGTTGGTAGAAGGTGAAGTGAGATACTTG GAAAAAGTTGTTCTCTTTGGAATCAATAAACAAAGGTTGGAGACTTGGGATAATGGTAGTTTAGTGATTCCTGATGGTCTTAGAGCTG CTCAAATTGAGGGAATTTCAAGAAGGATGATTGGAATGATAAGAGGTGTATCAAAGCTTCCAACATACAGAAGGAAGTTTCGACAGG TTGTTAAGGCATTAGTCACTCATACATTGGAGAAAGATGCTTCAGAAAAGGCTTTGGTTACACGTTCTCTTGATATAGATGTTTCTGAA AAGGCTTTAGTTACTCATTATTTGGAGAAAGATGTTTCAGGAAGATCCAGTAGGTCTATAGTATCAGTTCCATCAGATGACAATGTTT AA

$>$ Contig353

TGTTGCCGCTCCTTTCCCCCACTGCAATTGCAGAGGACGAGGTCGAGCAGCCTACGTGCGCAGAGGCTGATGCTTCTTTGGCGCAGAA CCAATTATTGTGTGTCGTCGCTTAGGCGGGGGCATCAAGATCGAACGTCTTTGCGATGGCACTCACCGACCTCCCCTCTTTCCTTCAAG 
ATTTCCCCTCGCGCCACAGTTATGCCGTCTTTGGGCCTTGCCTCAGAGCTCCGGCTAGCTGCTGACCGAGGGTTTCCCGGACCGAATG AATGTTGGATGATTCAGACTGTAATTACGACCGTGCATGACGGGTCTCCCACTCTCCTCGCCAGGAATCTCATTCTCTTCCATTAA $>$ Contig365

GTCACGGTCCCAGTTGGGACTACAGTTGGGGTTGGGGCTCCTCGCCCGGAAGTGGATACGGTTACGGTTCAGGTTCAGGGCATTTTCC TTTTGGTGTTGGTAAAGGTTTTGGAGTTGGTTTTGGAACCGGTACTGGATTTGGATCCGGGTATGGTTATGGATCGGGGGGGGGTGGT GCTCATGGTGGTGGTTTTGGAAGTGGTTCTGGCGGCGGAGGTAAAACCAACCACGGATAA

$>$ Contig381

ATGGATGCTGAACACGTGTGGAGCGTGGGAGCGTGCAGCAGAAGAAACGCTTCATCCATCAACGATAATCAAATCGTCAACGGAATA ATGCTCCGCTTCCGACCGATCGCACCAAAACCAGTCGCCGGAAACTCAACTACCTCCGGAGAACGAACTCCGACAATCCAAAACAAC AGTGTTTCTCCAGGTAAAAGAAAAAAGAGAAAGTACGTTAGGATTCGGAGAAACGAGGTGAGAAAGAACGGTGATAAATCATCGGA AAAAGTGGTGGAAACAACCGAAGACATGGATAGCGTCGCGGTTGTTACGTTACAGTTGATGCCGGAAAAAGAAACTCCTCTCGCCGG TGGTTTGTGGTGTAAAAACGTCGATCTTAACTTAACCGTTGAAAATATTCAGATCTTAGATAATCCGAAACCGGATCTGGAAAACGGA GACGCCGGGAAGATTTTAGATCTGGTTTCTCCGGTGGAGAAAACGGTGGTGGAGTCGTGGATAACGGTGGAGAGCGTGACAG $>$ Contig 482

ATGCTACCATTGAACAACATCATCTTTCTTATCTTTCTCATCATCACTTCCACTGCGGCAACAGGAAGAAAGCTAAGTTTCGATATGTC AAGCGGCAGTGCACCGACGTCAAGTGGTGGTAGCGGGACAGGTCACGGTCCCAGTTGGGACTACAGTTGGGGTTGGGGCTCCTCGCC CGGAAGTGGATACGGTTACGGTTCAGGTTCAGGGCATTCTCCTTCTGGTGTTGGTAAAGGTTTTGGAGTTGGTTTTGGAACCGGTACT GGATCTGGATCCGGGTATGGTTATGGATCGAAAACCAACCACGGATAA $>$ Contig574

ATGCAAAGAAAGATGCAAGGATACCCACAGCAACAGCAATGCTATGGTAGTGATGATGGCATGTGGATGGAAAGTAAGGGACAAAT GCAGCCACAGGTCCATCACTACCAGGACGATTCTTGGTCTAATGATAACAACAACCAGAAGCAACTATTTCCTGCAGCTATGCACATG AAACCGGCCGCTAATTTTGGAGGAAACAACCACCATATGTATCCTCATCAGGAAACCATGCCTGCTGCTGCAGTTGGTTATGGTAATC AAATGCATGGAAATGGTGGCAAACAATTCCCTTATGGTGGTACTCATAATCATTCTCCAAATGGTGTGAGGCCATTCAATCTTGAAGA GTATGAGTATGAAGCATACAAGGAGGAGCATGTTGGTGGTTCAAAGAAGGATGAAATGAGATATGAGCGACATGGATATGGAAATG GAGGTGATGTTCGCTATGTCAATTCATATGAATATAATAACAACTTCAACGCCCGTATTAAGCCTCATGCTCATGGACCCCACAAAGT CAACTGGACACTAAAGGGTGTCTAA

$>$ Contig583

ATGGCTTCCACTAAAGTGATGGTTCTTGCAACAATGTCGGTGCTCGCCGCCGTCCTATCCGTCGCCTCCGCTGCCGAGGCTCCGGCTCC TAGTCCTGTATCACCAGCCACCGCAATTTCGCCATCGTTCATTGTAGGTTTTCTCGCCGCTGTCGCTGGTCTCGCTTTTGGATCTAAACT CCAGATCTGA

$>$ Contig590

ATGGCGAAGAAAGTAGCAGAGAGAGCAGTAACAGCAATGAGGGGTTTACTGAAGGAAAATGCTGCAGACGGTGAAGATGGAGTTTC AGAGCCACCGTCGGGTCCGGGTCCCGGAGAGGGAGTATCAGGTTCAGGAGGAGGGGTAGAAGTGGAAGGAGCAGGTGCTTTGGATG GAGTACAGCACTTGGAGCCTGTGCTAAACAAGATGTGGCTAGTAAACTAA

$>$ Contig610

ATGGCAGGTATTGCATCCGGCATCTGCAACCTTGCTGTTGGCGATAATCGACGACGACCATTGCAACCACAATCTTCTCCTCCTCCTCC TCCTGCGCCGCAACAGTCATCAGAAGAGTTCGCCTATTCAAATAGTGCAAGGCAAAATATAAAAGGTCTTACAAATCAAACTGGCTA TGTCAAGGGAAATGCTAATGGAGTTATCAACTTTGGTACCTTGACATCCTCTGCTGCTCAGAGGCAATCATGA

$>$ Contig612

ATGGGTGGTGCATTTTGGGGGACACGGGTGATGGAGATAGTGAAAAAGCATGATTCTGGAGGGCTCGTTTGGAAGAGAATCAAGCTT ACCACCACTCGTAAAGCCAATGCCAAGAAACGTCTTCTCCGTGTTTGGCAGAATGAAGCTGTCCTCAAGGCATGTTTTGAACCATCTC CTTCAGCAAGTTCTTCCGGCAGTGCCAGCAAAGCTAGTAGTTAA

$>$ Contig618

ATGGCGAAGAAAGTAGCAGAGAGAGCAGTAACAGCAATGAGGGGTTTACTGAAGGAAAATGCTGCAGACGGTGAAGATGGAGTTTC AGAGCCACCGTCGGGTCCGGGTCCCGGAGAGGGAGTATCAGGTTCAGGAGGAGGGGTAGAAGTGGAAGGAGCAGGTGCTTTGGATG GAGTAGATGCTGGTGTTGGTGATGGAGGAGAGGTTGCCGGAAAAGAAGCTGGTGCAGGAGAGGTTGGAGAGGGAGTAGGAGGTGCT GTAAGTGGTGGTGTTGCTGGTGGTTGA

$>$ Contig634

GTAATGGTGGTGGTTTAACAGTTACAAGCATTGCTGACAACAACAACAATACATGTCCCCTTTATGTTGTTCAAGAGAAACAAGAAGT ACAAAATGGTCAACCAGTTACTTTCACACCTTATAATGCTACTAAAGGTGTGATTCTAACTTCAACTGATTTAAACATCAAATCCTCTT TCACAAAAAAAACTACATGTGCTCAACCACCTGTTTGGAAGCTTTTTAAAGTTTTGTCAGGGGTGTGGTTCTTGAGTACTGGGGGTGT TGAAGGTAATCCAGGTTTTCAAACTATTGGCAATTGGTATAAGATTGAGAAAGCTGGTAAAGATTATGTGCTTTCTTTCTGTCCTTCAG TTTGTAACTGTCATACTTTGTGTAGGGAACTTGGGTTATATACTGATGATAAAGGGATTAAGCACTTAGCTTTTAGTGATCAAGTTCCA TCATTTAGGGTTGTGTTTCAAAGGGCTTAA

$>$ Contig634_b

CACCTCAACCAGTACTTGACATCTCAG

$>$ Contig641

ATGGCTGATGACAACAACAGAGGCTCCTCTGCTTTGGATGGTTTCACTCTGAATCCTCTGCCTTATCCTGTTCTGTTAATCTTAGCAGT GATCTTCATCTTCCTTGGTACTTCATGGTACTTTTCTTATGAAGAAGTTGTTGAAACTGCTCAAGAACAATTGGGTTGGGTTTTATTTGT TGTACCAATCGTGCTTATACTTATAGTTCGTTTGGTTTCATCAATGGAAGATTCAGGTTGGTTTTCTGGTGGTTCTGGTTGGGAAAGGC GCAGGACAACACAAGAAAGCACCTCTGGAGGGAGTTCTCCTTGGGGTGTGGCTGCTTTGATTGTTGTTTTGTTGATATTGGTGCAGTT TCAATCTGTGTTTCTTGATAGTTGGTTTTATTGA

$>$ Contig657

ATGGCTTCTCAATCTCAAACTCAAACTCAACTTAAGCTAACCATTTCAACATCATCACCCAAAAGTGACAGTGGTATAAACGGCGATG TAGCGGCGTCGTTTCTCAAAGGACAGTGCCTATGTTCTCCAACGACACATGAAGGCTCTTTCAGGTGTCGTTTACATCGTTCAGGGGT ATCTTCGTCATCTACACCATCTTGGATGAAACGGACCAAATCATTGCCTCCTAATCATAAAGCTGTTTCTGTTTCTCCACAGTGA $>$ Contig670

CATCATGGTGAACACAAAGGGGAGCAGCCTCATGGGTATGGAGAGCACAAGGGAGAGCACCATGGTGAAGAGCACAAAGAAGGGTT CATAGACAAGATCAAGGACAAGATCCATGGTGAGGGAGAGGGTGGATGA 
CACCACCAGCACCACCAGCAACTGCAGAGGTGGAGGAGGAGAAAGAGAACAAACCAGTTGAGCCAGTGGAAGCTCCACCAGCAGCA GTAGCAGAAGTTCCAATTGAGACTCCTGAAGGTTAG

$>$ Contig701

ATGGAGAACAACGGCAGCCTTTTATCAAGGGAGAAGATCGATCAGATGGCTACCTGGGTGGGGTCCACCGTCTCTTCCGCTTTCTTCT CTTCCTTGGAACGCTTTTCTTGTGTTAACGTCGCCACTTCTGACCCTGACAACGACGAGGACGACGACGATTACTCCGTCACAACAAC CACAACAACCGCCACCACCACCGCCGACAGCAACAATCCTAATCCTGACGTTCAGGTCAACGGTCATAATACAAACGACGTCACCAA TCTCCCCGTTTGA

$>$ Contig767

ATGGCGAATCAAGCTGTTGCAAGATTCTCAATTCTTCTCCTCCTTTCTTTACTAGTCAACATCGCTTCATCCGCTGATTCGCCGGCTCCG ACGCCGCATTCATCCGTTAAAGTTTCGAACTCTTCTCCGGCAAGTTCACCGCCATCTCCATCTCCGGCGACGCCTCCTCATCCTGATTC GCCACCAGCACCTTCACCTGAGAACTCACCTTCTCCTTCTCCGTCTCCAGATGAGGCGGCTGATACCGGAGTTAACCACACCGGCGTC GGCGATAGAGGCGAAAAATCAACAGAAGGAGGAATGAGTTCTGGCAAAAAGGCAGGGATAGCGGTTGGAGTAATCGCAGGAGTAA GCGTGGTTGCATTGGGAGCGATAGTGATGAAGAAGCGTCGAGAAAACATTCAGAGGTCTGAGTATGGATACGCAGCAAGAACAGAA CTTCTCTAG

$>$ Contig773

ATGGCAGGCAAGGGAGAAGCAAATAAGTTTTCACCAACTGAGGCTTTTGAGAACGCAAAGAGTGCAGCAGGAACAGCAGGAGCAAC TGTTGTGGAACAAACTGGTAAGGCAGCAGATGCTACAGGGAATCTTTTAAATAAGGCTGGTGATTCTCTCCATCAGTTTAAGCCTGCA CCCAAACCTGCTGAAGGTCCTGTAGCTAAGGCTACTAAGGCTGCTGGAGACTTGTTTAAGAAATGA

$>$ Contig800

ATGTCGATTGGAGAGACTGCTTGCTCCTACGCCCTTCTCATCCTTGAAGATGATAAAATCCCCGCAACTGCTGACAACATCACCACCT TGTTGAAAAGTGCAAAGGTAGAAGTAGAATCGTTTTGGCCCAGCTTATTTGCCAAGCTTGCTGAGAAGAAAAATATTAGGGATTTGAT TTCCAGAGGAGAAGAAGAAGGAAGAACCAGCAGAAGAGAGTGA

$>$ Contig805

ATGGCAAACATGGACATCATAATTGAAGCTCCAATAATAGCCACTGAACCTGTAACAGATCCGGCAGCTCCTGCATCGGGACCCGGT GCCGGAGCGGGGGAGAGACCGAGGTCTTGGGCGGAGGCCACCGCGGCGAAAACAAATACAAAAAAGAAGGAAAATAAATTGAAAG TCGTACCAGATGATGCCATTGTTAAAGTATAA

$>$ Contig805_b

GTTGGAGÄGATTTGCGAAATGATATCGACGACACTAATAGGCGTGATATTTAGGAAGTGGTGTTTAGGAAGATATGATCAGGTA

$>$ Contig867

ATGGCTAAAGGTACTACTTTTACTACCCTTCTTTTCTTTCTCCTAATAATAACTTTCCTTTCAATTGCAATTTCAACACCAACAAATTTC ATCAAATCATCATGTAGCACAACACAATACCCAACACTTTGTGTTGAATCACTTTCTACCTATGCATCAACAATTCAACAAGACCCTC ATCAATTAGTCCAAACAGCTATATCAATAAGCCTCAACAAAACCCAATCCACTAAAGATTTTGTTACAAGGTGCAAAAATATCAAGG GTCTTAAAGCAAGAGAATATGCTGCCTTACATGATTGTAGTGAAGAAATTAGTGATAGTGTTGATAGGCTTATCCGTTCATTAAAAGA GCTTAAAATTTGCAAGATCAAAGGTGAGGATTTCAATTGGCATATAAGTAATGTTGAGACATGGGTTAGCTCAGCTTTGACTGATGAA ACAACTTGTGGTGATGGGTTTGGTGGAAAAGCACTTAATGGGAGAATTAAAGCTTCCATTAGATCTAGAATGATTAATGTTGCTCAAG TTACAAGTAATGCTCTTGCACTTGTCAATCACTATGCTAGTAATCACTAG

$>$ Contig885

ATGGAGGCATTGAAGATGAATGTTTTCGTAGTAGTTATGGTTGCTATGTTGGTAATGGTAGCTAGTGGTGTTTCAGCTGCTGATGCAC CAGCTCCAAGTCCTGTTTCTGATGCAACTACACTATTTGTTCCAACTGCTTTTGCTTCTCTCATTGCTCTTGCATTTGGCTTTCTCTTTTG A

$>$ Contig887

ATGGCATACCAAAACCGGAAACATAACAATCATGGTTGGAGTCACATGCATGAGGTTAATGAATTCGCAGCGTATCCGAGGTATCGA TATCAAGATCGTCGTGTTGAAATATACGAGGAACCTATAGCCGAGGTAGAACAATATTCCTACGTCGAGGCTCGTCGTGAGACTGAG ACCGATCGACGAGGTACGAGTTTTGGCAATCAATATGGTACTTATGAGAGTGTTGATCAAGAGGCAGGTGCTTTTATTCAACATGAGC ATAGGAGGATGGAATTAGCCAAACTCATGTCATCAAGAAATGGTAATTAA

$>$ Contig906

ATGAGCAGCAATCCAGCATTGCAAGTGAAAAACACGCAAATCCAGAGAAACTGGAAAGTTGAACAAAAGCTGGACCTTACGGACAC AATCAAAGACGGAGCTCGCCTTTTCTTCGTTGACGAAGGAATACGCCGAAAGCTTCTTCTTGCTTTGACAGAGGAATCAAAGCTTCAT ATAGAAGAGCTTGTTGACGTGTATAGGTTGGTTGAAGATCAGATAGATGTACCCTCAGTGGCTGTTGAAGTAGTTCGAGATTTTCCAA TTGTCATCAGGGATCTCCTACTTTCATGGAGTCAGTCCGTCTTATCCGATAGATAG

$>$ Contig914

ATGGCGAAATACGCATCTTCCATATTACAAACCCTGAAGCGCTTCATGAAAAAGCCATGGGAGATAACAGGACCCTGCGCCCATCCA GAGTACCGATCAGCATTACCAGGAGCACTTGAATACCGTGTTTACAGCCCCGCCACCCCAAAGGAAAAACCTATTATCCCTTCTTCCC TTCCCGAAACCGTATACGACATTAAGTATTACACACGTGACCAACGCCGTAACCGACCTGCCGTTGCCCGCACAATCTACAAGAAGG CCGATATCGAGAAACTCAAGAAGGAAGCTACCTTTAACGTCTCCGATTTTCCACCGATTTATCCCAACATTATCGTTGAGGAGGATCA GGATGCTCGGGGCGGTGGATATCAATCATAG

$>$ Contig953

ATGAAGAGCAAGGGACAGAGCCAGAACACGTTCGTGAGAATCATGTGTTCACCAATAAGAGCACTTGGAAAAGCAAGAGATGCTTA CGTAAGAAGCATAACAAACTGTGGTCAAAATATGCATTATGGAAATCCAATGGATGCTGCTGGAAAATTCTCTGCTTTGTCAAGAAG CCAAAGTGCTGCAACATCAAGAAGATCATCAACAACATCATCATTTGGAGATAATAGTGAAGATTTTGCTGAACTTGTTAGAGCTGCT TCAGCTAGAACTTTGGTTAATAGAATTGACATGGATTTGGTTCTAAAACAACAACAATTGAAGAAATCAAATAAAGGGTTGCCTAAG TCAAGTAGTGTTGGAATGGCTAAGATTGATGAAGATATGCCTTTTGATTATTCTAGTGTTGAGAAAAGGGGTGGTGTTGGGATTGTTC ATGATTCTTATCCTAGATCTAAAAGTTATGCTCTTGGTAATAGGAATGTTGATTTTTGA

$>$ Contig969

ATGGCGGGAGGCCATAATTACAGTATAAACGTCGACGAAATTAAGGCAGAGGTGAAAAAGATGCTTCATGATGGGCAAGAAGTAGT AATTATTGGGGGTACTCATGCACTACATCACCATGAGAAGCTGACAATTGCGGTTTCAAAAGCTATGCGTGGACATTCTCTTCAGGAA ACCAAGAACGATGGTCGTTTCCATGTTCATACCAAGACTTATCTGGATGGTGCTATTTTAAAAGAAGAGATGGAACGTTCTGCTGATG TTCTTGCTGCTGGATTGCTTGAAGTTGCTGACCCATCTCTTTCAAGTAAATATTTCCTCCGCCAGAATTGGATGGATGAATCAGAAGGG 
TCAACTGATTCAATTCTTAAGCATAAGCCTCTTTGGTCTTCATATAACTCAAAACATGGCAAGAAAAGGAGAAAGAATGTAAAGAAA CAAGGGGGTCTCCAACCAACTTATGGAACAAGAGTAGTTCCTGTTTTTGTGCTATCATTGGCTGACGTGGACCCAAATCTTATGATGG AGGATGAAAGTATGGTGTGGACAAGCAATGACGTTGTAATTGTGCTTGAACATCAAAATGATAAGATTCCTTTGAGTTATGTTTCAGA AACATATAGAAGGCATGCG

$>$ Contig973

ATGGTACAGAAAGTTGTTAGCATGATGCCAGCTCACTTTCAAGTCAATTACAAAGAAACAAGTAACAACGACAAGAACCTCTTGTTA TGCAGTTCTGAGATATTCTACGTACCTCAGCGCTTCGTCAGTGATTTTGTTGAGCTTGTTAACTTAGTAGACAATCTAGAGATCCATCA GAAGGTTGCAATTCCTATGTTCTTTGTCTCCATGGATTCTCCACAGAATTTTGATCCTATTCTTGATACAACAATCTACAAGAAAAAAC CTCCAACCACTAATTCCTCGACTCTTTATTCAGCTAAAGTTCCTGCTGTTCATCCATGGAGTGTATCAACTGAGCAAGAATTTATAAAG CTTATCAGAGTAAGGGCTGAAGGGGATCCACTCCTTATGGAATTGGTTTAA

$>$ Contig1017

ATGGAACATGATGAGCATTCAGAATCACAATCAAATGGTGGAGATGTATCTTCAGCCACAGCAGTTCTCCTAGGAGCTCTAGCTCCCG GTGTTAACGGACCTACATGGAATACTTTAAAGTCGGCGTTTTTAATGTTGGGTCTATGTCTTGCTGTTATGTTGGGCTTAGCATTCTCTT CCAGTGATTCATCGTTGGTGCTCCATGTTGGGTTCCTTGTTCTAATTTGCCTTACCCTCTTCTTCCTCCTCAGCTGGTTCCTTGCAGAAA CTGGTTTAGTCTCTATTGAACATCAAATGCGAGAGATGGGCTTGAATCCTAAGGATGCTCTAGAGACAAACAAAAAGAGTGAATAA $>$ Contig 1055 ATGATCTTCTACCGGAAGGGACCTAAGCCACCCAAGAAGGGTCAGCCCGAGAATGCAGTTTATGATTTTGAAGACAAAATCAACTTT GCTGTTTTCCCCTCCCTTCAGGGTGGTCCTCACAACCACCAGATTGGTGCCCTTGCTGTTGCTTTGAAACAGGCCACGACGCCCGGATT CAAGGCATATGCCAAGCAAGTTAAGGCCAATGCAGTTGCACTTGGAAATTACTTGATCAGCAAGGGATACAGCCTTGTCACTGGAGG AACTGAAAATCATCTTGTCTTGTGGGATCTTCGCCCTCTTGGCTTGACCGGCAATAAGGTGGAAAAACTTTGTGATCTATGTAACATC ACTGTGAACAAGAATGCTGTTTTTGGTGATAGCAGTGCCTTGGCTCCTGGAGGAGTAAGGATTGGTGCCCCAGCTATGACTTCTAGGG GTTTGGTTGAGAAGGACTTTGAGCAGATCGCAGAGTTCCTTCACCGCGCAGTGGTTCTCACTTTAGATATTCAAAAGGAATATGGAAA ACTTTTGAAGGATTTCAACAAGGGATTGGTGAACAACAAAGCTATAGAAGACCTCAAAGCTGATGTTGAGAAGTTTTCGGCCTCATTC GACATGCCTGGATTCCTAGTATCTGAGCTGAAGTACAAGGATTAG

$>$ Contig1092

ATGCAAGCTTCCGATGAACCCTACGTCATGTCAAGGGTTAACTCGTTATACTGCCTTCTTCAGAAAGACCCTTCAACCACCGAAGACA AAACTGTTCACAATACGGACGATGATAGAAAAGTTGACGCGAGTAATAACTCTACTCGATGCGAAACTAAAATGGTTGATTCGGAAA GCGAACAACGTGATGATGACGATGACTCTGGTAATCAGCAAGAAATTGGCTTGTCTAGGAAGGAATCAGCTGGAGAATTGCTTATGA ATCTTCCAAGGATTGCTTCAATGCCACATTTTCTGTTTCCTATGTCTGAAGATTCTGGTAGACAAGTTAGATAG

$>$ Contig1104

ATGACTCAGTACGGTATGATGCATTCAAAGCAGTGTAGGCCATCCAACTCTGGAGCAAACCTTGGTCGCAAACGGGTCGGGTTTAGCT TGTCTCCAGCGGGTATTATTGTTTTCGGATCTTTGGCGGTGGTGGCGACGGATGAGGAAGTGGCTTCCGTATTGGGCTTTAGATTGAC ATTATTTGCTGGGCCCATACCGAAGGAAAAGCACCATTTCTGGGCCTGCGAGATCTCAAAGTGGCTGAAGATAGACATAAACATTTTT ATTGCTAGGAAGAGGTAA

$>$ Contig1115

CTCTTGGGATTCAAGAACTTGCTTTTTCAAAGGACGAAATTGTCGGAGCTTGGAAAGACAATCACAACCGAACAGTTTATGTTTGTGG TTGTGACTTACCTGGTTGTCAAGAAATATATATTGGTTGGAGTAGACCAAACACTACTAATAATGTAACAAACTTTGCAATTGGTTCA ATCACTCCTGGAGAAGGAGATCTTGATGATTTAGAAGGTCTTTATTGGAGTCTTACTGATGGTTCTGAAGAAGAAGATAGAATCAAGA AAGGAGACTTTTGGCTTGATAGAGAACATGGTCATTTTAAAGGAGAAATTGATGATGATGATTGGGTTCTTGAAACCAGATTATACGA CAGAGAACCTGGTCCATCTATGTGTCTTAAAGCAGCTCGTTTTGAAGGAAGACCAAGAGTTCAAGGTCAATGGCAGAGAACTACTTCT ACAAATGATCTTCTTTATATTAACATTTGTCCTGATGGAGATGGCTTCTTCAGACAAAGTGTTTCTGGTGTTGGTGGTAGACTTTATGG AGAATTTGTAACTTTGGCCAATCTTACTTGGTTTGGTAGAAAGTTCACAGTTAACTATACAACAGGAGAAGAAGTAAAAG $>$ Contig1184

AATGGTTCAAAGCATGAGGCAGGCAATGACTGGCTGCAAGATGGAAACTGCCCAATTGCAAAGTCATACCGTGCTGTTAGTAATGTC CTTCCACTTGTAGCGAAGGCTATTCAGCCACCTGCCGGCATGAAATACAAGTGTCCACAGGTAATAGTTGAGGCCCGGGCGGCTATA GCACGCACTGCATTTGCAAAGAATCTACGCCCTCAGTCCTTGCCTACAAAAGTTCTTGTGATAGGGATGATGGGGATGGCAGCTAATG TTCCCTTGGGTATATGGCGAGAACATACCAAGAAATTTTCACCATCATGGTTTGCTGCTGTCCATGCCGCTGTTCCATTCATAGCAATG CTTAGGAAGTCTGTCTTAATGCCTAAATCAGCCATGGCATTTACCATTGCAGCATCGGTGTTGGGCCAAGTTATCGGCTCAAGAGCTG AGAGATACAGGCTGAAGGCAATTGCTGCAAAAAAGTTGTCTGCAATAGAAACTTCCGATGTTGGTTCGGTCCAGTTACCTGTGGTTAA ATCTAAAGACAGGCACTGTGGTGATGATGCTATGAAATGGAATGCAGCTTCCCTTCAGCTGACAGCAACTTCATCAACTGATGTATTT TGCTGA

$>$ Contig1184 b

GCTTTTCCATGGGAGTGATGGTGTAGTTCCTTTATCTGAGAGATCTTTTCAGCGTGTAGAGAAAGTGAACCATGAACATCCAAAATCC CAATTCAACCCTTTGGCTGCAAAGGCAGCAACTATTAGTCTATCATCATTTGGATTCGGCGGGTTCGGTTTTGACGCATTTTCTGAGAA GTGGAAAAACCAGAATAAAAAATCCAAGTCATCAAAAAAAGAACCTTCTTCACAG

$>$ Contig1184 C

ATGACTTATACGGGCCAACGGGATTTCCTTAGGTCGTTTGGGGTCATCAGGACCGAGAGTTTCTCAGTGGTTGGCTGTGGTTTTCCTAG CCT

$>$ Contig 1185

GCGACAAGTTGAGTTCAGAGAATATCCACGATGGAAAACTCGTTGTTCCCGTGGAGGATTGTAAGGTGATGACTATGGACCTTGACC ATCCCGATTTGTTATTGGGCTCAACGGATCGGTTTGGGCCTGATCCAATAACCGTACAGAAACAAGTTAGGGTTTCCGATTCGAAAGA TGGAATTTACGCTGGATCAGCGTTTGTTGCGTCTCCTCCTCCGAGTTCCGTTCCTGTACCCGGATTTTTGGGGAAAAACGGTGTGGCCA CAAGTGATTTGAGACGGATACTACGACTTGATTTGGAATGA

$>$ Contig1185 b

ATGGGAACCGCAATTCTTCGTTCCCACGATTGCCTTCAAGGTCGGTTTCTCCCAAACGATGACTTGTCAATCCCTTCCTCTAAAATTAG GTCTCGAAAAAACTCTAACCCCAACCCTAATTGCAAATCCTATGTCAATCAGAATCGTCGCCGTAAGCGGAGCCCGGTGGCCCCCGCT CAGGCCAATTTCAACGATCGGAGGAGATCCGGTGATCGTACGGAGACGCTGTCGAACGGTGTAGCCCGCG

$>$ Contig1190 
GTGGTCCTGGTCGCAAAATTCCGGAACAGAAACCTCTTGAGAATACTTCTTCGGTTTTGTATATTGGTAGGATTCCACACGGGTTCTAT GAGAAGGAGATGGAAGCTTATTTTGGACAATTTGGAACTATTAAGAGATTGAGAATTGCCAGAAATAAAAAGACTGGAAAATCAAG ACATTTCGGGTTCCTAGAATTTGAATCTCCTGAGGTAGCAAAAATTGTAGCCGATACTATGCACAATTATTTGCTTTTTGAACACCTTC TTCAAGTTTTTGTTGTACCTTCAGAGGATGTTCATCCCAGATTATGGAGAGGATTCAATTACCGTTACAAGCCCATTGACTCTCTCCAA ATTGAACGAGAGCGACATGACAAGGAAAGAACTTTGGAAGAACACAAGAAGTTGGTGGATAGAATTGTAAAACGTGACAAAAAGCG ACGTAAAAGGATAGAGGCTGCTGGCATTGATTATGAATGTCCCAATATTGTGGGTAATCTCCAATCTGCTCCAAAGAAGATAAAGTTC GAAGACTGA

$>$ Contig1195

ATGACGAAAATCGAAGCTGCGTTGAACAATGAGTTGTATTTAACACCTAAGAAGAAAAATGAAGGCGAGTTCTGGTTTTCGGATGGT CTTTTTGTGGACTATGATGTTACTGGTATTTTGGTTTCTGAACAATGTAAAAGTACATCATCTGATCTTGAACCAAGTTGGATCGGAGA TGAAAAGACTCGGCCGTGGTGGCATGCTTCGGGAAAAGATGACTTGGCTTCCTTGGTTGCTAAGAAATCACTTGAGCATATTGAGAAT TGTGATCTACCAGAGCCTTACATTAAGCCTTTTAGGAAAATACCGAGACATCAGCCGACAGGTATCGACAATGACAAGAATTATGTAT CGTCCGATGCGAACGATTGTACAACTACAACCCTGACTTCTGGATGTTCATTTCAGGATTCAGATAGAATTTTCAGTTCCAGTGAAAA CAAAGACTATGATTCAAGCGACAGCAGCGATATTCGCATGGATTCCGAGAACACGGCCAAGGCAAAGCTACTGAAAGCCTTGTGCCG ATCCCAAACTCGGGCGAGAGAGGCGGAAAAGGCAGCACAAGAAGCCTGCAATGAGAAGGAACATATCTTGAGTCTATTTTTGAAAC AGGCTTCACAACTATTTGCTTACAAGCAATGGCTACATGTTTTGCAGCTTGAGAATCTATGTCTTGAACTCAGAAACAAAAACCAGCC ATTGATGAACAATCTCTTTCCCTACCGGGAAAAACAGAACAGGAAGAGCCGTCGAAAGATTAGGAGTCGAAGACGGCGGATCGGAA AATGTGAAGTTGCTTTTGCTGTTGGTTTGGCTCTTGTTGGTGCTGGTTTGCTTCTTGGTTGGACTATTGGATGCATGTCTCCACCCTTTT GA

$>$ Contig1 199

GTGATGATAGGTGCAAAGCTGGCATAGGCTATGCTGTTGCTGCTAATTATTGGGGACAAGGTATAGCTACAAAAGCACTTAAGATTG CTCTGTCTCAAGTGTTCATTGATTTCCCTGATTTGTTAAGGTTGCAGGCTTTTGCTGACGTGGACAACACGGGTTCAATAAGAGTTTTG GAAAAGGCTGGGTTCCTTAGAGAGGGAGTTCTTAGAAAGTATACTTATATTAAAGGTATCATCAAGGATTTAGTTGTGTTTAGTTTTTT GTCAACGGATGAAATTCCTCTCATTCATTAG

$>$ Contig1223

CCTATGAGGTCAAAGCCACACCACAAATTGAAGGTTTTAGCACTTCAAAGCAACAAAGGAAGCCTAAGTCCATGTATGGTATGACGT TGGAATCAAGCAAACCATTACCAAACACTCAAAGAACAAATTTAGGATTGCATGTTGTTCAACATACAACGATGTCAGAGAAAACAA AGACTGTTTATGGGGGAGCTAATAATGTGAAGGGTCCACATAAGGGAAAAAGTAGTGCAAGCACCAATTCAATCAAATTTTCATCAT TGTTCATGGCTGCACTAAGCCATTTCATTATAGCAACGATTATAGTTGGTTGCTTTTTTTGA

$>$ Contig1234

ATGGGTGGAGGTGCTCACGCTTTGAAGAGAATCCCTCGCATCAAGTTCCCCAACAGGCACCATAAATCCTCTGGTTCTACCTCTGAGA CTCATGTTTCGCCGTCAACAAGCGGTGGTAGTTTATCATTCTTTTCAAGTTCAAAAGCTTCAACAACGACTACATTAGGAGGAAAGGC CTCTCTTCAGCCCAAAAGAACTCCAGTGACCAACGAAGAGATTGAAGCAGTTTTGTTGGGTGGCTGCTTCTAA $>$ Contig1239

ATGGCAGGAATCATTAACAAAATTGGTGAGACCCTTCATATAGGAGGGGATAAGAAAGAAGGTGAACACAAAGGAGAGAGCCATGT TGAACAACAACATGGGTATGGTGGAGAGCACAAAGGAGAGCAACATGGTGTGTATGGAGGAGAGCACAAAGGAGAGCAACATGGTT TGTTTGGTCATGGAGGTGAGCACAAAGGAGAGCAACATGGTGTGTTTGGAGGAGAGCACAAAGGAGAGCAGCATGGTGTGTATGGT GGAGAGCACAAAGGAGAGCAACATGGTCTGTTTGGTCATGGAGGAGAACACAAGCCAGAACAACATCATGGAGAACAAAAGGAAG GATTTTTAGAGAAGATCAAGGACAAGGTCCATGGTGAAGGTGGAGAGGGTGAAAATATCAAAAAGAAGAAGGATAAGAAGAAACGT GGTGAACATGGTGGTGAACATGGCCATGACAGCAGCAGCAGTGATAGTGATTAG

$>$ Contig1241

ATGTTGATAGAAGCTCTTGATGTCTATCTTTTAGGAACAGTAATGCTGGTATTTGGAATGGGTCTCTATGAGCTCTTTGTCAGTAATCT TGGTAGTGCAAGCTCTCTGCCAGATCAAAAACCTTCTGATAGATCAAATCTATTTGGCTTATTCACTTTAATGGAACGACCGAAATGG TTGGATATAAAAACTGTGAATGAACTGAAAACCAAGGTTGGTCATGTGATAGTGATGCTGCTTCTGATTGGGTTGATTGACAGAAGTA AGAAGGCTACTATACAATCTCCTTTGGATTTGCTTTGCTTCTGTGCTTCTGTCTTTCTTTCTTCTAGTTGCCTCTTTTTGCTGTCCAAGCT GAATGAGCCTAATTAA

$>$ Contig1243

ATGGCCACCGGCGCCGCAGATGGGTTTCTCCGGCCAATTTATGAAGGTTGTATCTCCGGTTACGACCACTGTGTGGAGCGTCGTCCTT ACCACCGTAACTGTGGTTGTGCACTTCATAGCAAGTCAAGAAAATTATGCACACACAAAATGCCAAGGTGTAACAATGTATCATATCC TATGAGGCGGGCATGGAGTGAAGGGAATTTAGTTTTGATGGCTTCTGTTCATTCTTCTCCTTCTACTTCCCCAGCTCCTCTTAGTGGTG TTAGGCCTCAACGTGGTTCCGTGGATCTAGATCATGATGTTGAAGTCGAGCTTAATAATAAAATTAGTAGTTTTATTTGA $>$ Contig 1259

ATGCGGAATTTATCCGAGTCAGTGGCAGTGCTGAAGGGAAATCTTGGAGCCATCCGTTCAATCCGGTATACTTCCGATGGACGGTATA TGGCTATAGCAGAGCCTGCAGATTTTGTGCATGTATATGACGTTAAAAGTGGTTACGAAAAGGAACAGGAAATAGATTTCTTTGGTGA GATATCCGGAATATCTTTTAGTCCAGACACCGAATCCCTTTTCATTGGGGTTTGGGATCGTACTTATGGAAGCCTTCTTGAATATGGCC GGCGCAGGAATTACTCATACCTCGATTTTATGATTTAG

$>$ Contig 1293

ATGGCTTCCTACACCGTTGTTTTTATGCTAGTTGCTACATTGTTGGTGAGTTCCACCTTTGCTCAATCACCGGCATCGTCTCCTGCTTCG TCTCCGACCAATGCTCCCGTTGCAACTCCTTCATCTTCTCAGGCTCCTGCTGTTTCTCCTTCCGCCAAGTCACCAGTTGCATCTCCTCCT GTGCCTGTCACCAGCGCTCCTTCTCCTTCACCCTCCGTCGTCGACTCTCCACCATCTCCTCCTCCTGCTTCCACTCCCGCTGGCGCTCCT GCAGTTACTCCATCATCGATTTCTAACACTCCAACAGAAGCACCAACCCCTTCTCCAAATGGTGCCGTTTTGAACAGATTCAGTGTCG CTGGATCTGCTGCTGTTGTCCTTTTCGCTGCTGCTTTGATGATGTAG

$>$ Contig1304

ATGGCTTCCATTGAACCCACCGCCGGCGGCCCAAAATACGCCAAAGAACATAGCTTTAGGTTTCGAGATCCGCATTCCAGAAACCAC GGACCAAAATGGCCGATACGGGTCATTGGCACGGCGGTGGCGGCGGCGGTGGCCGTGATCTTAGTAGTGTTAGTGGTCCGTTGGCTG AGGAACAGAAGATCGGCGGCGGAGGCTAACGTGGAGTTGGCTGTCATTCCGGTGATGCCAGTTGAGGAGGTTATGGAGTCCGGTCCG GCTTCTTCTTCTTCCTCAGTGGATGCTGCTGGCATGGACGTAATGGTGGAGGGAAGATAA

$>$ Contig1326 
ATGGCCACTGTTGAGGTTGAAACACAACAAGTACCAACAACTGTGACAGAAAATGAAACAGTTGAGGTAACCAAGGTCGAGGAAAC AACACCAGCCTCCGAGGTTCCTGTCTCAGAGGAAACAACACCAGCCACCACCGAACAAACAAACAAAGAAGCCGTCGAAGAAGCAG CAGCAGTTGAGAAAGAAGCGGAAACAGAAGTGCCAGTTGAAGTTGGGACTACACAAGAAACAGAGGAAGACAAACCAAAGGTCGA GAATCCAGCACCCGAAAAAGTTGAGGAAGTGAAAGAGGAAGCAACAACTGAGGAAGCCACTAAGGAAACTACTGAAAAAGAATCC ATAGAAGAAGCAGCACCAGTGGAGGAGAACAAACAAGTTGAAACTGCTGTGGAAGAAGCCACCACAGAAGCATAG $>$ Contig 1357

ATGTCGTTTATGAGAGGAGATTTGCTTTCTCGAACCAGAAAGCTTGTGAAGGGTTTGGCCAAGGCAGAACCTGTGTGGCTCAAAGCCA TGGAACAGGCACCACCAGCGACATTTCCTCGGCCTGTGGGGAAAATTCAGACCATCACTCTTCCTGAGGATGTTTATGTAAAGAAGTT TTACAAAAAATACCCAGATTCAAAATACCATGATCCCATCAAGTTTTCTGCTATTGAACCCCCTCCATCTCGTCTATTCGCCTTAAGGG TTCTGGAGTTGAAAGAGCAAGGCATTAGTGAGGAAGAAGCAATGGATGTAGCAGATATGGAATATATAGCAGAGAAGAAGGCAAAG AAGAAAGCATATGCCCGTCTGAAGCAAATAGCACGCCTTCAAGGAAAGAGACTTCCTCCAAACCCATATCCTTGTCCTATCAAAGAG ATTCAAGCTGAGGAGAAGAAATAT

$>$ Contig1392

ATGGATAATAAATCGGGTTTCAATGGAGATAGCCACTTTAACCGAACATTCTCCTCTTATTCTTCCGCTTCCCAAAGGGATGTCCGTTA CAGCTGTGGTGCTTGTGGGTATGAGCTTAACCTATCCTCCTCAAACCGGAACACCACATCCATCGGATCTAAATACGGGAAGTCCATA AAGCGAGGTGTCATATCATTCTTCAATATTGATGACAGCAGATTTACCCAGGTTGATGAGATTCAGTGTGTGCCTCATTTTCATAAAC ATTCATGGGGCTTGTTTCGCCGAACAACAAAGCTTCTTTGTCGCAAGTGTGGTAACCATATTGGAAATGCATACAATGGTTTCACTTC ATCCTTACCTCTCGTGTCAGACGGAGCAGAGTCATCCCCTGGCTCCAAAGTGTCCGGTAGTGTAAAATATGACATTCGCCTTCGGTCG TTACAGCCTTCATCGTCTGAAGAATCTGGAATCCGTGCATTAACTTGA

$>$ Contig 1408

GGCGCCTCAAGGTTCCTGTGCATACAGTTGACAGGAAATTACTTAATTCAGTCTAA

$>$ Contig1408 b

GTTTACTTCCATCACCGTCCACGCCGACTCCTTTCGCCGCCGATCTCAAATTTCGGTTCCCAAGCATGTCACCGGCGATTCAAATTTCG ATTCCTTCCTTTCATTCCTCGAACTCTCCTGCCTTCTCTCTTCGGTTATCGTTTCAGCCAGCGTTGCTGTGATTGCGGTTTGGAAGAAGG AGTTGTTTGTAGCAATCGGTAATAGAGTTTCTCCGTGGAGTGTGTTGTTGTTGGTGGTTGGTGTTTTGACTGGTGCGTTGATTAGGAGA CGGAAATGGAGACAAACGGTGGTGGACGGTGGGTTTCCTGTTTCGGAGGTGAATTTTTTGCAGAGGATGGAGAAATTGGAAGAGGAT TTGAGGAGTTCTGCGATGGTTGTTAGGGTTTTGTCGAGGCAGCTTGAGAAATTGGGGATTAGGTTTCGTGTTACTCGGAAGTCTTTGA AGGAACCTATAACTGAGACTGCAGCTCTGGCACAGAAGAATTCCGAGGCCGCAAGAGCATTAGCTATGCAATCAGACATTTTGGAGA AGGAGCTCGGTGAAATTCAAAAGGTTTTACTAGGCATGCAGGAACAACAGCGAAAACAACTTGATCTCATTCTTGCAATTGGGAAGG $\mathrm{CAG}$

$>$ Contig 1447

TGATGTGCAATGTGATACAGTACAGAACATTACTGGGTATCTCCGCTGAGGTTTGTTTTGGTAATCAAGCATCCTGTTCCTGCCTTGAT GGAGGGTGCACCATTCCTGCTGAATGCAGCAAGTTAGCCATTACAGGGGTTGGAACAGAGATCCTTGATCAACAAACCTGA $>$ Contig 1460

GTTATCCTGGTCAACCTAGAGCTAGAGGCGGAGAAGTGGCCAAATTTGGTCGTCCGGCCAAATTACGGAGCTCGACTTGGTGGATAA GGTTTTTTCTTCAGACAAAATTAGAAGAAAATGAAAGTGCTGGAAAGAAGTTTGAACAGGCTTCGGCCAAGAAGGCGTACAAACCAG CTTGTCAAGTTTTCCACCTTAAATCATACCCATGA

$>$ Contig1478

ATGGAGGATTCCACCGACAAGAGAAAGCGAGCTAGAGACGAGTCAGGAGACTCACCTGACTTGCCCGATTCCGAAACTCACCGAGTT GACTCACATGAGACTAAGATTCGACGTGTTAACTCATGTTCTGATGTTAATTCGTCAGAGTGTCAGCTTACCCGAGTTGACTCGGCCG AATCGTGTCTTAATTCGGTTTTTGACTCCGGTGTTCAACTTCAAGATGACATTTTTCATATACTTGATGATACAGATAATGTTCCTGAG CGTGAATCAGTGATGGGTCTTGACTCAGTGATGAAAAGCTTCGAAGATGAGATTTTAGCTCCGGGTTTGAATTCGGGTCAAGTTGACC CGACTCAGACGACCGGTTCCGGAGAGATGGAGATGAATTTGGGTTACTTGTTTGAAGCTTCCGATGATGAACTTGGGTTGCCACCGAC GGTGGGTAATGGTGATGAACCGGGTCGAGTTGAACCGGAAATTGTGGACCTGACCGGGTTCGTGGGGTTTGAGGATGATTTTAACGG TTATGATGAGTTTGGTTTTGGAACAGGGTTGTTGTCGGAGTGTGACGGTGATAACGGTGGTGCCGGAGGTTTTGTAACGGTGGATGGG TTGTTTGAATATGCGGAACCGGCGGCGGATGTTTTGTGGCGGTCGGAGTCGTTACAAGCTATGTAA

$>$ Contig1482

GGAATGCATCTGCTCCTGGTGGGGATACTGGTGTGGAGTTGAATATCCCAGAAGGGATGGAGGGGCCAGTGAAAGTGAAGGTTCTTA CGTTTCCAGATAATTTAGCTCATTCTGAAGATGAGGAAGGATGA

$>$ Contig1493

ATGCCAAGTCCTAAGAACCAACGGAATCACCAGATCGAGAATCAACGGAAGGGAAGGTTGGGTGAAAAATCATCATCTTTTCACGGA AATAACGCCATGTCTACCGCCACAATTCGCCGTCCGAAAACCGTTCCTGACTTATTATCTTACCGGAGCAACGCCGCATCGGCGTTAG AAGGTTTTCCTAGACAACTGCCGAAGTTGCTTCTGAAGGTGACGGTTCTAGGTAGCCTTGGTCCTGTTCAGATGTTGATGAAGCCGGA ATCAACCGTCGGCGATCTGGTGGAGGCAGTGGTGCGGCAGTATGTTAAGGAAGGTCGCCGTCCGATCCTGCCTTCAAAATTTGCCTCC GATTTCGATCTTCACTATTCACAATTTAGCTTAGAAAGTTTGCATAGAGAGGAGAAAGTAGCGAAACTTGGATCTAGAAAATTCTTTC TGTGTCCGAGAAATACTACCCCTGCAGCGGAGGGTATTTCTGGAAATAGAAAAGATGGTGAAGTAACGACGTCGTTTGCTTCGTGTGC TAAAGAGGTTGATAAAGTGCGCGAAAGTTTAGGTTGTGGTGGTGGTGGAGGTGGTTTTGGTTGGTTCAAGCTTATGCAATTCATGTTG TGA

$>$ Contig1495

GTTATTAGCAGTGTGGGTGATCTAGTTGGCATAAAATACTTCCCTGCTGAACCAATGAAGCAGCAGCAAGATGGTAAAACTGTGAAG TTTGAATGA

$>$ Contig1540

ATGGCAACTCAGGTTTTGAACCCTCACGACTATTTCATCATACCTCAACCACCCTCCTTCTCCCGCCGCTGCAACTATTACGGCTACAA CAACCGTACCACGACCTCCAGGTCTCACCGGAAACATGTTGCACGACCAGACCTGAGAAAACGGAACGATAAGAGTTCCTTCCAACT AGAGACAGCGTCGGTCTCGAAAAGATCCAGCGCCGATGATTCAGCGACTGGGAAGAGCAGCAGCTCGCCTGTGGAGAAGGTGACGA TTCTCCGGAGAGGCCAGTCGCTGGATTCGTCGTTGGTAGCGGCGAAGAATGACATGTACGCTGGATCTGCGTTCGCGATGTCTCCGTC ACCAAGCGCGCTTCCTCTGCCGTCTTTTATGACAAAGAAGCAGTCGCTAGAGACGTTTGACGACTCCGCAACGCGAGATCTGAGGCGT TTGCTCCGAATTGATTGA $>$ Contig1558 
ATGAGCAACTACCAGAGAGCACCTCAAGACCCTTATCCTCCACCACCCTACGGATCACCTTACCCTCCACCACAGGGCTACCCCCTAT CACAACCTCCACCTGGCTACCCATCAGCACCACCGCCACCATCCTACGAAGGCTATCCTCCGCCACTGCCTCCTGGTTATTCAACCTAT CCATCGCGTCCTCAACATGAATCCTACCAAGGCTATTTCAACGATGGCTATCCTCCTCCACCTCCTCCTCCCAATTATCATTGCCACCA AGTCCAGCATCATTGTCACGATGATAATAACGCCGGCTTTTCTTCTTTCTTCCAAGGCTGCTTGACAGCGCTTTGTTGCTGTTGTTTGTT AAAGGAGTGTTGCTTTTGA

$>$ Contig1579

ATGGCTTCTCTCCTCCTCCGCCTTCCAATCGCCGCCGTGATCGTCCTCATCGCGCTATCCGCCTCCTCTGTCACCGCTCGTCCATGCCGG ACCTTCATCATCTCCTCCTACTCTTTCCGTAACCCTTCCTCCAACACCTTCGCCACCATCACCGAGATCCGATCCATCTCCCCTCTCTAC ATCAATGACAAACCCTACGAAATCTTTATTGATCGCCCGATTCAACACAACTTACAATTAGAAACCCAGTCTCAAGGCGCGTCGCACC CACGCGGCCCCTTAGGGTTTTCCACCGACGCTTACGATTTCTCCTCCCTTCGCGATCGCACCAAGGATATCCTCAGCGTCGCCCTCGCC TTGCTATTCGGCGTTGGATGCGGTGCCTTAACCGCCGCCACCATGTACTTGGTCTGGTCCGTCTTCACCGCCCGCCACGAACTCCGCGC GGCCGCTTACGGTGAATTCTCCGACGACGAGATTGAAAGCCCCAAGAAAATGGGATATGTCAAGATTCCGGCGGCTGAGGTGGCTGC TGCTGCTCCCGCACCTCCGGCCAAGGATTCGGTATGA

$>$ Contig1593

ATGGGGAAGGGGAAGAACGCGGAAAATACGGACGGTGGCATGCCGATGCCACTTCCACTTCCGCCGCTTCTCCGAACCCTTATTTCA TCCCTTTTCAACACCGCCGCCGATAACAACAATTCCTTCCTCTCATTATCTTTCAATTACAAACTCTTCCAAACCCTTCGTTTCATAATC CTCACTTTTTACCTTTTTCTCCTTCGTTTCATTCCTTCTTTCTTCTTCTTCGAAAATTACGAAAACGCCGTCTCTAAGCTCAATTTCAGCC CTAATAAATACTCACACGATAAAAGAAACGACACCGCAATTGGTCGCGCTCTTTCTCAGCTTCTTTCTGCGTTAAACGATATTCCTGTT AGTTCCAGAAAATACGAAGTTGTTCGATCGTTAACGGAGAAGATTATCGATGATAATCACAACGACGGTGTTCATTCGTTGCGTGAGG TTAATCGCGTTGCGCTTGCAGCGGCGTTTGGGAGGGCGTTGAGGCAGCTTGAAGGGAAGGTGTCGGAGTGTGAGAGTATTATTATAG GACGGTGA

$>$ Contig1613

ATGGGGAGTTCTGAAGGGTTAGGAGCATCGTCGAAGCCTTCTACTCAGGCACCACCAAAACAATACGGTGTTACGAAGCCAATTTCA ATGGCTGGTCCAACAGTGCTTGATTTAAAAAAGACTCAACAGTTGGAGAAGTTTCTCGCTGATTCAGGTTTATATGAGAGCAAGGAG GAGGCTTCCAAGAGAGAAGAGGTCCTTCAACGACTTGAACAGATAGTAAAAAGTTGGGTGAAGCAGCTTACTCGCCTGAAGGGATAC ACTGATCAAATGGTGGAAGATGCAAATGCTGCCATAGTCACTTTTG

$>$ Contig1622

ATGGGAGGTGGTAAAGACAATCATGATGAATCTGACAAAGGAATTTTCTCACATTTAGCACATGGGATGACTCATGGTGCTCATGGTT ACCCACCTGGGGCTTACCCTCCACCACCAGGTGCATACCCTCCACCTGGACATGGCTACCCTCCACAAGGGGGTTATCCTCCAGCTGG TTATCCCCCTGCTGGTTATCCTCCTGCTGGCTACTCTCATGGTGGCTATCCTCCGGCAGGTTATCCACCTGCTGGTTATCATGGTCCATC TGCTCCTCATGCTCCAGGGCCTTTTGGGCACGGGCATGCCGGTATGGGTGGAATGGGTGGATTGCTTGCTGGTGGTGCTGCGGCAGCA GCTGCTGCATACGGTGCTCACCATATTTCTCATGGTCACCATGGACATGGAGGATATGGTCACATGCCCCATGGGAAATTCAAGCATG GCAAGTTCAAGCATGGAAAATTCGGCAAGCATGGGAAATTTAAGCACGGAAAGTTTGGCAAGCATGGTATGTTCAGGAAGTGGAAGT GA

$>$ Contig1639

ATGGTGCAG

$>$ Contig1639 b

TTTCAAATG̈GTATTATACAACAAAAAAACAAATCACACCAACAACAACATTTGTAAAACAAAAAGCAAGAACAATTCTCATCAACAA CATGTTGAAGATGATGAATCTGGCATGTGTTCTCCTCCTTTATGGTCAACAAAGAATACTCACAACAAAAGCAACAATTATAAAAGTC TTTCACCTGAATCAAAGACACAAGCAATTGAAAGAGGACAAAAGGAGTTAATGGAAATGGTTAAGAACATGCCAGAGTCATGTAAT GAACTCACTTTAAAAGATCTTGTGGAACATCATTCTCCAAAGGTTGTTGAAGAAAAGAAGAATTTGGGCAACAAAAACACACGCAAA AGAGAAGGAATTAGTACTAGTAATAGTAATAATGGTAGAAAGATTGATAAGAGAAATATTGGGAATTTTGATAGTGGTGGATTTTAT CTCAAAATGGTGTTTCCAATTTCTTTGGGATCAAAGAAACATAATAAGAAGAAAGAGTTATTGGTTAATAATAATAGTGCTTCTTCAA AGGTTTCTCCAAGAACATCGGTTTCTGATGGATTTGTTAATAATAATAAAGAATGGTGGAAGAAGAAGAGTGTTTTGGAATGTGAAG GTGTCGAGAGTAATAGTGGGGGTGGCAATAGCATGAAAAGAGTTGGCATGAAATGA

$>$ Contig1722

ATGGATCGTAACAGTGTTCTCACCCTCGCATTCATCTGCATTGTCGTCGCCGGCGTCGGAGGTCAATCTCCTTCATCGGCTCCGACAAC ATCACCAACCGTAACAACTCCTACTGTTTCTCCCGTCACAACCCCTTCCAAACCTAAATCACCCGCTCCTGTATCTTCTCCCACGTCAG CACCACCAACTTCTTCTCCTACTGCTGCAAATGCAGTTGCAACACCAGCTACGTCTCCTTCTTCAAATGCCGCTGCTCCAGTTCCGGTA GCTAAGCCTCCAGCATCGTCACCACCGGCGGTTACTCCGGTGAGTTCACCACCGGTACCAGTGCCGGTGAGTTCTCCACCAGTTCCTG TTCCAGTGAGCTCTCCTCCGGCAAATTCTCCTCCTGTACCAGCACCAACAACTCCGGCACCGGCAGTTACACCAACCGCTGGAGTTCC CGCTCCAGCTCCGAGTAAATCAAAGAAGAAGGTAAAGAAAGGGAAGAAACACAGTGCTCCGGCACCGTCTCCGTCGTTGTTAGGACC TCCAGCACCGCCGGTAGGTGCTCCTGGACCTAGTATTGATGCATCGTCTCCTGGTCCGGCTTCCGCTGCGGATGAGAGTGGAGCAGAG ACTATCAGGTGCTTGCAGAAGGTGATAGGATGCTTGGCTTTGGGCTGGGCTACCCTTGTTTTTATCTTCTAG

$>$ Contig1724

GAGTGAGTATGAGGTGAAACCTAGCTATGGAAGGAGTGAGTATGAAGAGAAACCTAGCTATGGAAGGAGTGAGTATGAAGAGAAAC CTAGTTATGGAAGGAGTGAAAATGTGAGTGAGGAAGGTGTTGAGTATGGTTATGAGCGTCGGGAGAGGCGTGGTGATGATGATGATG AGGGTTATGGACGTAAGAAATATGGAGACAATGGTTTTGATGAGGATGAGGGGAGGAAGAAGCATCCCCCCAAGAGTCCCCATCGC AAGAGTTACGATGACGAGTAA

$>$ Contig1736

ATGGCTGATTTTCAACAAAAACTTGATGCACTAAAGGGTCTTGTTGGTGTGCTAAATAGCATGGGTGATGATGGTCATGAAAATGGTG ATTTTCCTTCAACTCCACATAATTACCATCCTACCCCTCAGCCAGAAAGGAAACGATCCTCTGGAAAATATAGCAACACTGGCCACCA AAATATAAAAGGTTTAAGTAATCAAACCGGTTTTACTGAAGGCAATGCTAATGGAGCTATCAACTTTGGTGATTTAAAAATGTAA $>$ Contig1770

ATGGGTATTCGTTTGCCATTTGTGGTTCTTCAAGCAAGACAAATATTCAAATCCACTTCGACACAACCAATTCATAGTAGAAAACAGT CCAATGTTCCAAAGGGGCACATGGCAGTGTATGTTGGAGAATTGCAAAAGAAGCGATTTGTGGTTCCAATATCATACTTGAATCATCC TTCCTTTTTGGACCTGCTTAATCGAGCTGAAGAAGAGTTCGGCTTTAATCATCCAATGGGTGGTCTTACAATTCCTTGCAAAGAAGATG CCTTCATCAATGTTACCTCCCAATTAAGTGCCTTGTGA $>$ Contig1786 
ATGGAAATAGTTGGTGAGATTCAACGTGCCATGGCTAGAGTGTGGCTATGGGATGGAAGGTCTAAGGCCCCACCAAGACTTCTTGAT GGTATGGCCGAGTATGTTGCCGAGTTAGCCGGATTCCGCCGTGAAATATTCTCCGGCGGCTTCGGTGAGTCGCCGGAATGTGAAGTTG GCCGTGATTTATGGTGGGAAGATAAGGACCCTACTCATGTGGCACGTTTGTTGCATTATTGTGAAAATTTTAAGAAGGGGTTCATCCA ACGGCTGAATGAAGCTATGAGAGACACGTGGCATGATCGTATG

$>$ Contig 1800

TCTCCCCCACCACCGTACAAGTACCCATCACCTCCACCGCCACCATACAAATATCCATCACCACCACCTCCAGTTTACAAGTATAAGT CCCCTCCTCCACCTGTTTATTCACCACCTCCACCACATTATGTTTATGCTTCACCTCCTCCTCCGGTCTATTCCCCTCCTCCACCGCATT ACATCTATGCATCACCTCCTCCTCCTTACCATTAA

$>$ Contig1827

CAAATAATTATATCTATTAAGAAGCCGTACAAATACCATTCTCCTCCTCCACCAGTTTACAAGTACAAGTCTCCACCACCACCCTATA AATACCCTTCTCCACCACCACCACCAAAAAAACCTTACAAATATCTCTCTCCTCCACCACCAGTTTACAAATCTCCTCCTCCTCCTTAT AAGTACCTTTCTCCTCCACCCACGTATAAATATCCATCCCCCCACCACCACTTTATAAGTAAACTACCTCCCCACCGTGTAAAATAA $>$ Contig1842

AGCCAGTATCCTCGATTGGTTGTTGATGACTTTTTGCCATTGCCTTCCAAAGGGCGCACTGACAAGGATGGGTGGTATCCTCCTGGTCA TGGGGATGTCTTCCCATCATTATCGAACAGTGGAAAGCTTGATGCACTATTATCACAGGGTAAAGAATATGTGTTTGTTGCCAATTCA GATAATTTGGGAGCCATAGTTGACTTGAAAATCTTAAATCATCTGATCGAGCACCAGAATGAATATTGTATGGAGGTGACGCCGAAA ACATTGGCTGATGTTAAGGGTGGCACTTTGATTTCTTATGAAGAAAGGGTTCAGCTCCTGGAAATTGCACAAGTCCCAGATGAACATG TTGGTGAGTTCAAGTCAATAGAGAAGTTCAAAATTTTCAACACAAATAATTTGTGGGTGAACTTGAAAGCAATCAAAAGACTCGTCG AAGCTGATGCTCTTAAGATGGAGATTATTCCCAATCCAAAGGAAGTTGATGGAGTAAAAGTTCTTCAGCTGGAAACTGCAGCTGGGG CAGCCATAAGGTTCTTTGACAAGGCTATTGGGATTAATGTTCCTCGATCCCGATTCCTTCCAGTGAAGGCATCTTCAGATTTGCTTCTT GTCCAGTCAGACCTCTACACCTTGGAAGATGGGTCTGTAATTCGGAACAAAGCTAGGACTAATCCTGAAAACCCTTCTATTGAACTGG GGCCAGAATTTAAGAAGGTTAGCAACTTCTTGAGCCGCTTCAAGTCAATTCCTAGTATTGTTGAGCTTGACAGCCTAAAAGTGGCTGG CGATGTGTGGTTTGGAGCTGGTGTTATCCTCAAGGGGAAGGTCAGTATTGTTGCCAAATCTGGCGTAAAGTTGGAAATTCCTGATGGA GCCAAAATTGCAAACAAGGAAATTAATGGCCCAGAGGACCTGTGA

$>$ Contig1843

ATGGCTGCACTTTGGTCATGTTGTTTACTTGCACTGCTTGTTTTGATGTCAGCTATTGTAACTGAAAGTAGAGTTGCAAGGAAGGACTT AGGTTTGGATCTTGGTGGTTTGGGGATTGGACTTGGAGCTGGACTAGGTTTGGGTATTGGAGGTGGTAGTGGCTCTGGAGCTGGAGCT GGAGCAGGTTCTGGTTCTGGTTCAGGTTCTAGTTCTTCCTCGAGTTCATCGTCTTCGTCTTCTAGTTCAGGGTCTGGTTCTGGTGCAGGC TCTGAAGCAGGCTCGTATGCTGGATCTCGAGCTGGATCGGGATCAGGAGGTAGAAGTCGCATTAAAGAAATAATGCATGAATGA $>$ Contig 1868

ATGGCTCTTCAAGCTGCTTCTTTGCTTCCTGCTTCTTTCTCCATTCCTAAAGAGGGTAAGGGTGGTGTTTCTCTTAAGGATTCCACCCTT TTTGGTCTTTCATTGTCTGAATCTTTGAAAGGTGATTTCAGCTCTTCTGCATTGAGGTGCAAGAGGGAATTGCGACAAAAGGTCGGTG CTGTGAGGGCAGAGACAGCAGCTACAGCAACTCCAACAGTTAACAAATCTTCACAAGATGGCAAGAAAACACTGAGGAAAGGCAAT GTTGTGATCACTGGTGCTTCTTCTGGATTAGGCCTTGCCACTGCTAAGGCTTTGGCTGAGACAGGAAAATGGCATGTAATTATGGCTT GCAGGGATTTCCTCAAAGCTGCAAGAGCAGCAAAATCTGCTGGAATGGCTAAGGAAAATTATACTATCATGCACTTAGACCTTTCCTC TCTTGACAGTGTTCACCAATTTGTTGATAACTTCAGGCGATCCGGAATGCCACTCGATGTGCTTGTTAACAATGCTGCTGTTTACTTGC CAACTGCTAAGGAACCTAGATTCACTGCTGAAGGCTTTGAACTTAGTGTTGGGACAAATCATTTGGGACATTTTCTTCTCTCGCGCCTT TTGCTTGAGGACTTGCAGAAATCTGATTACCCTTCAAAGCGCTTGATCATTGTTGGCTCAATCACA $>$ Contig 1878

ATGTCATCGCACGGGAACGACCCACGTCAGCCATCGGCGGGGAAGCCATACGTGGCTCCGGTGATTGCTCCACAAGACCTTCCGATC GACTATGCTGGTTTCATCGCCGTCATATTCGGCGTCGCAGGCGTCATGTTTAGGTACAAGCTGAGTTCGTGGTTGGCTCTTATATTCTG TGCGCAATCGATTGCTAACATGAGGAATGTTGAAAACGATCTCAAACAAGTTATGATGGCTATGATGTTTTCTATAATGGGATTGATA ACAAACTACTTTGGACCCCCTAGACCCGGTGGCAAGCAAAGCTGA $>$ Contig 1878 b

ATGGCTGGTTTACTAAGGAATGTATTGGGTTTGAACTATGGTGGAAACAATGGCACAGTTGATGCATTTAACAACAAATTGGGTACAC AAGATTACGATGAAGCTGAATTCAACACTGGTGCTGAAATTAAAAAAGGAAGTTACCCAACTCATAGCAACAATGGAACCAAAAAG GCATTCAACAACTCATGGGGAGGAACTCAGAAATTCGGAAAAGCTAAGTTTAACACTGGTGCCAAGATTGGAAACTAA

$>$ Contig1879

ATGTCAAGCTCATGCGTCAACTGCAACTGCGCTGACAAGAACCAGTGCGGGAAGGGAAACAACTACGGTGTGACCATTGTAGAGACT GAGAAGAGCTACTTGGAGACTGTTGTCATGGATGTTCCAGCTGCTGAGCATGGTGGGAATTGCAAGTGTGGTTCGAACTGCACTTGCA CTAACTGCACCTGCGGCCATTAA

$>$ Contig 1880

CTCACCACCACCACCACCAACAAAGAAACCATACTACTACCATTCTCCACCACCACCAGTTTACTCACCTCCAAAACACCCTTACCAT TATCACTCTCCACCACCACCGAAGAAACCTTACAAGTATCCTTCACCACCACCACCAGTTCATCATGTTCACCCTAAACCCTATTACCA TTCCCCACCACCACCAAAAAAGCATTACAAATATTCCTTTCCTCCACCACCAGTGCAT

$>$ Contig 1942

ATGGCTTGCGTCGCCGGCACTGCCGCGAGATCGATATTCCGGTCATCCTCCGCCGCCCGCACCGCTTTCCGTATCGGTTCAGAAGCTA AAACAGCTCGTTCTCCTTTCCGAATCGCCTCCAATAAGCCTCTTTCGCAATCCGCACTCAGGTGTCCTGTTGAATTGAGCTTCTGTGTT GAATCGATGTTGCCGTATCATACGGCAACTGCTTTTGCGTTGATGACTTCAATGTTAGTAGTTTTTGAACGCAGATACGGTTGGCTTCC TGAAGGTAGCTAA

$>$ Contig1944

ATGGAATCTCATGGAACATCATGGGCTGACCAATGGGAAAATGGCCCAGACCCTGTGAGTGGTTCCAACCAATCCAAAAAGAAGAGC AACAACATTCTAGGCAAGACCAAAACAGTGGCCTCCACTGGCGTCCAGAAGTTGAAAGAGGGTACCTCTGTTAGTCTCAAATGGATC AAAACCAAGTATAACAAAACCACCAACAAACATTAA

$>$ Contig 1950

ATGGCCTCTGTTCAGTGCTATAAAATCTGTGAACAAAGCTGCCAACAACACAAAACCCAGCAGCAACATGGTTCCTTAGGACAGAAG GTAACTGACTTGTTCAAAGGGCACCCCAATGAGGGAACACAAACCCAATACTGTAGCAAGAAAACTGAGGTGATTTCTCAGTCAGGA AATTTAGTTTCCAAAAGTGAAACTAAAAAATGCAACCAAACATTCAACAACAGTGGTGCTTCTAGTACCACCGTGAAATGCCAGGGG 
AGAAATAGGAGGCAGCACAAGAGAAACAACTTGTTCCAGAAGATCAAGGATGGCATATCAGGTCACAGCAGTGACAGTAGCAGTGA TGAGAGTGACAGTGACAATGAACATTGTCACAACAGGAAGAACTGA

$>$ Contig1962

ATGGCGGTTTCAGGTTTAAGAACTGCTACTTTACCATTCTTTCATCATCATCATCATCACCGTTTCTCACTTTTACCTCCTTCCTTCTCC AATCTCAATCTTCATTCCACCTCAAAACCACTCACTCTCTTCGCTCGCTATGCTCAAACTCAGGACAACCTTTTCTCCTCTCGTCGATTC CAAGATAGTATCGAAAAGCTACCGAAGCTTGTGGAGGACATTGTCCAGACATCTGTGGATACAGGTCCTAGGGGTGTTCTAAGGTTG GCTCAAGGTGTTCAAGCTTTCATTGGGGTTGGTCAAGAGTGGTTAACTGATGTATCAAAGTCACCAAATTCATCTGCTGGATTACTGA CAGAAATGCAGCTTGGTCTACTGTCCCCCTTTTATTTGAGGAGGTTGTTTGAACGCTTGGGGGCAACCTACATCAAATTAGGACAGTT CATAGCATCCGCACCGACGTTGTTTCCTTCTGAGTATGTACAAGAATTCCAGAATTGTTTTGATAGAGCTCCTCCAATTCCTTTTGAG $>$ Contig1967

ATGGGGTCTCTAATGGCCTCTATCACTTTAACTCTTCTATTAGCCATACTATCTCTCACCACCTTCCCATCACAAATTTCAGCAAACAA TTACTTGTATTCCTCACCCCCACCACCCAAGGTATACCCCCCAGTCTCACCACCACCTAAGGTATACCCTCCAGTCTCACCACCACCTA AGGTATACCCTCCAGTTTCACCACCTCCTAAGGTGTACCCCCCAGTCTCACCACCACCTAAGGTGTACCCTCCAGTTTCACCACCTTAC CACTACTCCTCACCACCACCACCAGTTCATCAT

$>$ Contig2012

ATGGCTAACTATGCTATACCCTATGTGTTGATACTTCTCTTGAACTTGAGTACTATACTCAATGTCCTTGCTTGTCCTTATTGCCCATAC ССТTCTCCTAAACCCCCAAAACACCATCCTCCTATTGTTAAGCCACCACCAGTTCACAAACCTCCTAAACCAACACCTTGTCCTCCCCC ATCATCATCACCTAAACCACCTCATGTTCCAAAACCACCCATTGTGAAACCACCAATCGTGAAACCACCGATCGTGAAACCACCGATC GTGAAACCACCGATCGTGAAACCACCGATCGTGAAACCACCGGTCGTGAAACCACCGGTCGTGAAACCACCGGTCGTGAAACCACCG GTCGTGAAACCACCAATTGTGAAACCACCAATTGTGAAACCACCAATTGTGAAACCACCCAATGTGAAACCACCCATAGTGAAACCA CCCAATGTGAAACCACCGATCGTGAAACCACCTATAGTGAAACCACCCATTGTGAAACCACCCATTGTGAAACCACCTATTGTGAAA CCACCCATTGTGAAACCACCCATTGTTAAACCACCAATT

$>$ Contig2037

ATGGGTGTGACAAAGGAACAAGTCGAGCCACCCCGGAAATCCAAACTCAACCCGCCCCACAGCCAAGTACTGGATACATCTGGAGG GTGTGGTGCAAGTTTTGTAGTTGAGATTGTGTCTGAACAATTTGAAGGGAAAAGGCTATTGGAAAGGCATAGAATGGTGAATGCTGC CTTAGAGGAGGAAATGAAAGAGATTCATGCACTCTCTGTAAAGAAAGCTGTAACCCCAGAACAGTGGAAAAAACTGCAAGAGTCCA ACCAATCAAATCCTGCTGCTTAG

$>$ Contig2049

ATGGTTTCTGCTCCACTCTCATCGTTAGCAAAACCGGGTCCAGGTGATAACGCATCTTCACTGGGTCCAGGAGCTCCTGTTGGTGGTG CAGGAGGACTACTCAATGTTGGTGATGGTGCTGGAGCACTGTGCTTCTTCTTTCCCTTCTTTGACTTGCTCGGTGCCGGAGCTGGAACT TCCGGTGTGGTCGGCGCCGCCACTGGTGCAGCTGCTGGTGGTGAGCTTACAGGAACAGGGGTTGGTGGTGAGGCGACCGGAACTGGT GCCGGTGGAGCACTAACTGGTGTGATCGCCGGTGGTGTACTGACTGGTGTCGCCGCCGGTGGAGATGATGCTGGAGGTTTGGTCGCC GGAGCAG

$>$ Contig2068

CCATACAAATATTACAAGAAGCCATACAAGTATCCTTCACCACCACCTCCACCAGTTTATACTTCACCTCCTTACATTCCACACCCAGT TTATCACTCACCTCCACCACCAGTTCATACTTCTCCCCCATACATTCCACATCCAGTTTACCACTCTCCACCACCAACTCCAACCAAGA AGCCTTACCTCTATTCCTCACCTCCTCCTCCTTACCACTATTAG

$>$ Contig2089

ATGGCCGATGATACTATTAATAACAGTTGCAAGAAACGAGTTCGTGATGACTCGGACGAGTCGGAACTTGAATCGCCCGAGGCGAAG CGACTCAGAGACGATTTACTCGAGTTTTTGAACGATTCCGATCCAACTCCTTCAACTCAGGAACTTGACTCAGTCATGAAGAGCTTAC AAGAAGAGATCTCTGCATCTTCTTCTCCTCCTTCTTCTCTCCGTTTGACATCAGATTCCGGTGAGTCTCAGGCTCAGATCGGGTACTTA TTGGAAGCTTCCGACGATGAACTCGGGCTTCCCCCGCCGGGAAATCCATCGGTTCAGGAAGGGAAGAAAGAGGAAACTGAGTTGGGA CGAGTTTTGTCTGACTCGTCTGGAATCGGTGAGTTATGGGAGTTTGAGAATCAGATCCTGAGTTATGACTCGTTCGAGTTAGGAACCG GATTTGGAAGTTATGAGAATATTACTGAAAACGCTGCGTTTGATGGAATTTTTAACTATTCCGATGTGTATTATGACACCAACGATTTT TCTGATCCGTGGCGGCACGGAACCTTGCCGGCACAATAG $>$ Contig2171

ATGTTTAGATCAATGAGTACTCGAAGAGGGTTTGGGACTGGAAGGTATGAGAGATTAGGAAAAGAATCAACAACCACAACACTTTTG AATGAAGAATTCAAGAGAAGCACAAGCTTGCCTTCTAAGGCATCCAATAATTCTTCAAGAAAAGTGGCTCTAGGTTCAACTTTTGGTG ACATAAACCTACAAAGAAACCAAACAAAAAAAGTTAGTAATAATAGTAGCAAGAAGAGTCACCCACTTCTTAGCTTTTTGGATCTTC GTAGGAAAAAGAAAACAACAGCTAAGCCTGAATTTGCAAGGTATCTTGAGTATCTCAAGGAAGGAGGCATGTGGGATTTGAATTCCA ATAAACCAGTTATCTATTACAAGTGA

$>$ Contig2 182

TTCTGGCCGAGGATGAAGACAGGTGGCTAGTTCCGGCGCCGGTTGTTTCTGTTGCCTCTGCGTCTTTTCGGACTCGATTTCGTAAGAAG CAGAGGAAGGCTGGCGCGCCGGATTGGAGGCCTTCGCTAGGACCGATCTCAGAGGACGTCGTCGTGCCGCCGAAAGCCGCCACGATT ACCCCCGGAAGGGAGGCGAAGAAGAAGACTTCCGCCCGCGGTGCCACAAAGGTTTATCGCCGTAGCTACAGGGACGTTTATCAAGGG TCATCATTTCCCGTGCCTACAATTATGCCAGCTTTTTTTCCAACGCCATTCATGTTTTGA

$>$ Contig2190

ATGGAAAAGCAAAACTTGAAATATCGAGGGTGGTGTCCCTCCAACACAAACAATTACGAAATCCACGAAGTAGGAGAGCCTGCACTT CGTAAGCGGCTAGATCGTCCTCGACGTGATAATAAGAAGGGTCATGTGGCATTCAAAACAGTGCTAGAGGAGGCCATAACACCACTA GAAGTGGCAGAGACTGAACGTGTAAATTCTTTTGAGAGCGTGGATCGACAAGCTGATGCGTTTATTAAGCTGGAGCACAGGAGGATT GAACTTGCCAGACTCAAGTCTTTGGGGTTTGCTTGA

$>$ Contig2214

ATGTATGCTCAATTGTTGCCTCTTGCTTTGCCTGCACCACCAATGCCTGGAATGGGAGGACCAGGTTTGGGAGGAAGCTATGGGCCTC CGCCTCCAGGAATGGGGATGCCCCAAATGCCTCCTTTTGGGATGCCACCCATGGGTAGCAGTTACTAA

$>$ Contig2245

ATGTCAGGAATCATCAACAAAATTGGAGAAACTCTTCACATTGGAGGACACAAAAAAGAAGAGGAACACAAAGGTGAAAAACATGA TCAACACAAAGGAGAAAAACATGATGAACACAAAGGTGAAAAAAAAGGAGAACACAAGGAAGGTATAGTTGAGAAAATCAAAGAC AAGATCCATGGTGGTGAGAGTCATGCATGGTCATGA $>$ Contig2268 
ATGTCTTCTCATAACATTGCTTTCTTCTTCCTAACCGTTTTACTTGCTACCGGCGCTCTCGGTCAAGCACCTTCTACCTCTCCCACCGCC TCACCCAAATCATCACCGGCTCCGGCGGCATCTACACCTACACCAAAAGCCACTCCTCCTCAAACTCCCACCGCCACTCCTCCTCAAA CTCCCACCGCCACTCCTCCTCCAGTTCCCACCGCTACACCTACTCCCGCTCCAGCCTCACCTCCTCAAGTTTCATCTCCACCTTCTCCAA CACCAACAACAACCACCGTCTCACCTTCCGAATCTCCGGTTGAGTCTCCGATCGATTCTCCTCCCGCTCCCGTCGCTCCTACTATTTCC CCCTCCGCTTCCGAACCTGCTACCGTCGGCGATGGTCCTGCTCTGGCTCCTAGCGGCGAATCCGCTTCCGTTAGAGTCGCCGTCGGTG GATCCGTCGCCGCTATTGTGGCATTTGGTTTGATGATGTAG

$>$ Contig2272

ATGGCTCAAGAGTACTTTGAACCTGCTAAAGGTGGTCTGATCATCGATGTTAGCTGTGGAAGCGGATTATTTTCCCGAAAGTTTGCCA ATTCTGGAACCTATTCTGGAGTCGTTGCTCTAGATTTTTCTGAAAATATGCTTCGCCAATGCAATGATTTCATTAAGAAAGATGACACA CTTTCATCCACTAATATCGCCCTTGTAAGGGCAGATGTTTCTAGGCTTCCCTTTGCATCAGGTTCAGTTGATGCTGTTCATGCTGGTGCT GCTTTGCATTGCTGGCCATCTCCGTCCAATGCTGTTGCTGAAATCACCCGTGTACTAAGAAGTGGTGGAGTATTTGTCGGAACCACTTT TCTTCGTTATACTTCATCAACTCCGTGGATTTTACGCCCCTTCAGAGAGAGGACTTCTCAAGGCTATGGCTTTTTAAAAGAGGAAGAA ATTAAAGACCTTTGCACCTCATGCGGTCTTACAAATTACTCATGCAAAATCCAGAACTCTTTTATTATGTTTACAGCTCAGAAGCCATA A $>$ Contig2284

ATGGAAATAAAGAGGTACTTAAGTAGACGTGGCTACCGGCGTCTCGACGGCAGCATATCCACCGTCCGACAGAAGAAGATGCAAGTC ATACAAATGAAAGGAAGTCCTCATAAGCATTGGAGGATTGGAACAAGCCCAAGGCTAAGATGGATGATGAAATCACCATTGAAATTG TTGACAAAAGTTAAGAATATTTATATGAACTTTATGTTTAAAGTGGCTGGGAATGTAGGAGGGGCTTTGAACACTGATAACAACAACC TTGGTGTGAAACAAATTCCACAAGCTCGTCAAAAGTCTAAAGGTTACTACTCTGGTGATGCGTTTGAGGCTAGGCTTATATTTGAGAT TTCCAAGACCTTGGTTGCTTCTCATGAACTATATTCCATTTAA

$>$ Contig2302

ATGGGCGAAGCATTGTTCGATCTCGAACAACTTCTCATGTCCAAAAAGGGAAAATTGACACCTCACGAGGTAAATATTCTTCAATCAT GCAAATCAAAAGCTACGAGGGATTTCACTGCTAGTTCACTTGTTGGGGGTGCTGCTGTATGGGCAGCAACTGGGAAACTCCGAACAG CACTTCGAGTAAACCTTTCAGCAGGAGCTGGTGGTTTCCTTGGACTGTGGGTATTCAGTAGATCCTTGTATTCTTCTGCTGATCATATT CTTACTCTGGATGGCAGTATATTACAGAAGGAGTTAGCAAATATACTGGTGACGAAGTACCAGAACAATCCTTCGCTAATGCAGCTTA TATCTAAGCATTTTTATTCGGAGTGGATTTTTGATGATTCAACCTCTAATACTCCTAAATTAAGGTGGCGATACCGTAATTTTTTTAGC GATAATGTAATCAATGGAAGCAGCAGCAGGACTCAGGATTATGGTTCATATGATAAATCGCAAGAAGACACTCAAAATGATTCCTAC GAAAAATCCCAAGGATATTCTGAATCCTATGAAAAATCCCAAGGCAAGTCTGAAAATATCACTGACAGCAAAAGAACAACTCTTCAG ACCAAGAATTTTTTTACAAATCCTGGTCCTGATATCGTGTCAGAGGTAGACCCTCTTGATTGTCTTTTTGGCTATGGTGCTCCAGTGGA GGAGATTTTTCATCCTAATACCCCAAACAAACCATCAGGGACGCATCATCGAGGGCATAGAAGATATCGCCGTAGGCGTCGGATGCG TGATCTTGATGACCTTTCCAACTCAGAGCATGCAGCACCTGTTTGA

$>$ Contig 2310

ATGGATTCAAAGAAAAGTGATGAAGAGGTTCATGTGGTTCTTGCTGTTCATAGCCAAGTGATCAAAATTAAGAAAGAAATTGAGAAA ATCAAACACCCATCATTGGAAGCTGAATTGAAGATGAGAAGATTCAGAGATGTTAATCGTATTCGTTCTCGTTCACCACTTGGTTTGG CTGAAAGGATGATCCTTCTTGGTCACTCTTAG

$>$ Contig 2335

GTTCTTCTGGTTGGTAAAGGAAAAATGAATGCATTAGCAGCTACCAACAGGAATTTTAAGTTAGCTTCTAGGCTTTTGGGGTTGGATT CAAAGCTTGAGAAAAGTTTATTAATTCCATTCAGAGAAATCAAGGTTGAATGTACTATACCTAAAGACGACGGCACGTTGCAATCTTA TGTTGGATTCAGAGTTCAACATGATAATGCTAGAGGTCCTATGAAAGGAGGAATCAGATACCATCCTGAGGTTGATCCTGATGAAGT GAATGCATTAGCACAACTAATGACATGGAAAACAGCTGTAGCAAATATACCATATGGTGGTGCCAAAGGAGGGATAGGGTGTAACCC TTCAGAATTAAGTATATCTGAGTTAGAGAGACTTACCAGAGTTTTTACTCAGAAAATTCATGATCTCATTGGAACTCACACCGATGTG CCTGCACCTGATATGGGAACTGGACCACAGACCATGGCATGGATACTAGATGAATATTCAAAATTTCATGGCTACTCTCCTGCAGTAG TGACCGGAAACCTATTGATCTTTGGTGGATCTCTA $>$ Contig2456

GTGGGGTAGGAGATGGTGGAGAAGGTGTGGTTCCAGATGGTGGAGGAGAAGGTGTGGTTCCAGATGGTGGAGGTGGTGGTGATTAA $>$ Contig2459

ATGGCATCAATGACCGCATTCAAGGCTTTCTTCTTTGCCGTTGCTCTACTGTCCGTCGCCGTTTCTGCTCAAGAGACGTCTCCTGCGTC GTCGCCTGCACCGTCTCCTGACGCCGGAGCAGCAGGATCTATGTCCACCTCAGTTGCCATGATCGGCGCCTCGCTCGTGTTGTCCATG CTTGCCGCGTTCAAGTTTTGA

$>$ Contig2462

ATGGCAAACATCGACATCATAATTGAAGCTCCAATAATAGCCACTGAACCTGTAACAGATCCGGCAGCTCCTGCATCGGGACCCGGT GCCGGAGCGGGGGAAAGACCGAGGTCTTGGGCGGAGGCCACCGCGGCGAAAACAAATACAAAAAAGAAGGAAAATAAATTGAAAG TCGTACCAGATGATGCCATTGTTAAAGTATAA

$>$ Contig2495

CTGAAGAGAGTAGTGTTCATCAAACTATCATAAACGATGCAAGACCTCACAGTGAAGTGGACACTTCTCAAGTGAAATCTCATCCTG AAGTATCTACCTGGCTTGGTGGTGACGTAAGCCCTGGTGGATTTGGATCTGGTATTAAGCCAACCAGATTGGGTAACTATGTGATGTC CTTTGACGGTGATACCCTCCAGAGATATGCAACAATAAGGTCTAAGGAAGCTGTGAGCATCATTGAGAAGCACACTGAGGCATTGTT TGGAAGACCTGAAATGGTTATTACACCTGAGGGGGCGATTGATCGTTCTAAGGATGAAAACATCAAAATTAGCTTTGGTGGTTTAAA GAGACTTGTTTTAGAGGCTGTGACTTTTGGTTGTTTTCTCTGGGATGTTGAGAGCTACGTGGAATCAAGGTATCAATTTGTCTTTAATT GA

$>$ Contig2516

TAATTTCATTCACATTTGTTTTCACAAATGCAGAAGATCCTCTTCTTGAAATTGGTGACCTTGATCGGTACAGTTTCCCTAAGGGCTTC GTTTTTGGGGCAGGATCATCAGCATACCAATTTGAAGGTGCATGGAACGAAAGTGGCAAAGGACTAAGTATCTGGGATACTTTCACC CATAATCATCCAGAAAAAATAAGGGATGGAAGTAATGCAGACCTCACTGTTGACCAATATCACCGCTATAAGGAAGATGTGAAAATT ATGAAAGACATAAATCTGGATTCGTACAGATTTTCAATCTCTTGGCCAAGACTCCTCCCACAGGGAAAGTTGAGCGGAGGCATAAATT GGGAAGGAGTACAATATTACAACAATCTCATCAATGAACTGTTGGCTCATG

$>$ Contig2531

ATGGCTGCTGCTCAATTCCTCACTCTTCTTCACTCTTCTTCAAGATCTTCTTTGCTCAAACCCTCTAGACCATTTTTCTACAACCCCATC AAGAATTATGGGGAATCAATCAAAGGAAAACAAAGCAGATTGATGGAAGAAAGGGCACCATCTACAGCTGAAGAATTTCTAAGAGT 
GGCAGAGGAGAAAGAAAAGGAAACTAAAAAAGTGGTGGCAAGTCAAACTATTGATAAAACAATTGATGCTGCTGAAGAAGCTACTA AGGGTAATTCAAGAATTGAAAATGTTAAGAATAGGTACAAGGAGGGGTGA

$>$ Contig2579

GATAGTGGCCTACCACGGTGGTGGTGGTAATGGAGGTTATGGCAGTGGCTCTGGGTATGGTTCGGGATATGGTTCCGGTTCGGGAGG AAATGAGTACCCTTAA

$>$ Contig2614

ATGGCCGCCAACAGATGGATTAGACCCGAGGTATTCCCACTTTTTGCCTCAGTTGGTGTTGCTGTTGGGATCTGCGGTATGCAACTTGT AAGGAATATAACCGGCAACCCTGACGTCAGGGTAACCAAGCAGAACAGAGCTGCAGGAGTTCTTGACAATTTTTCTGAGGGTGAGAA ATATTCACAACATACCCTAAGGAAGTTTGTCCGCAACAAGAGCTCTCAGATTATGCCATCCGTTAACAACTTTTTCTCTGATCCAAAAT AG

$>$ Contig2616

ATGATGAGAGGGAATCAAGATCAACAATCGAAGATGATCTGCGAATTATCGGCTCTGGTTTTCAACCTTCTACAGTTTTCTCCGACGC CGTTGTCTTATTCCGATCGATCTCCGATCGTGCCGGTGCCTCCGTCTGAGTCACCGTTGAGGCGTGCAGGTCAGATTACTCCGGCTGGA TTTGCGTCGTTGCTTCTGGGAATTTCGGTGGCTCTGATACTTTGCGGATCGGTTACTTTCTTCATTGGGTTTATGCTGATGCCGTGGGTT CTTGGATTGGTTATGGTGTTTTACGTTGCTGGTATCGTTTCATCTCTTTCTGTTTTGGGTCGTTCCATTTTTGCTTCGCCTCGCCGCGTAA GGATGTTCCAGAGTGGAAATTGTTATGAGGAGATGAATATGGCATTGAGCAGTATGATACTG

$>$ Contig2626

ATGGAAAACCAATCCACAACAACCCCATCCACGATGCAGTTTCTCATCCTCGAACAAGTTCAGTTCTTGAAAGTAAACGACGATTCTC TTCTTCAATGGGAACTCGTCGATGTCGTTGACGCCGAAGAAGAATTAGAACAACAATCAATCGATGATGATGGGGATTCTTTCCTTTC TCGTGACCCAATTGAAGGTATTCGACATCGCGTTCTTCATCTCGATGCACATGTAGAAGATCATCACCATCACCGTGATGCGGATGCA GATGGGTTTAGTGAAGAACAGGTTAACCGCTTGTTCCATGGGATATTGGGAACAAGTTAG

$>$ Contig2654

ATGGCATCATCAACTACTACAATTAAATTATTATCTTTCTTTTATGTCATCGTCTTTGCCGCCGTAGCCTCAGCACAAGACCTTAGTCCT TCGTCGGCTCCGGCACCAAGTCCTGATGCGGGAGCTGCCGGATCCATTACAAATCCAGTGGCTATGATTGGAGCTTCGATCGTGTTGT CTATGTTCGCCATCTTGAAGAACTGA

$>$ Contig2667

ATGGCGAGGCAATGGAAAGAGATAGGGCTGACAAGACACCAAGGTGGTAGTGGCAACTGGGTAGAAGAGTTTGCAA

$>$ Contig2667 b

ATGGAGTTTCCAAAGAATCAACCAAAGCCTCCAACACCACAACCAACTATGCCAATGGTTCTGCATGCGCTGCTGCAGCGTGTGGAG CAGGCTGTGGCGGAGGCTGTGGGGGTTGA

$>$ Contig2670

ATGTGGCCTCACATAAACAACCGTATACTAGCATCTTTGATATTGTACCAGATAACCATGTTTGGGTACTTTGGGGTGCAGAAATTCT ACTATGCACCATTGTTGATTCCTCTTCCAATACTGTCATTACTCTTTGGTTTCGTCAGCGCAAAGAAATTTTATCCCGCTTTTCAACATC CGGCACTTGAGATCGCAGCTCCAGGGCTCAAGGAAGTTCCTAACATGGAATTGATTTTCAGGTCTTTCATTCCACCAAGCTTGAGTTC TGAGAAGGTTGAAGATGACCAGTTCGAAGATGCACGGTCTCAAGTATCAAGATCAACATCATTTGTTTGA $>$ Contig 2727

CTTTGGGACAGTATAGGATTTGGTTTAGGATTAGATTTCGATGATTACGAGGACGGAGTTTTTTCCTCAAAAAACGACGTCGTTAGCA AACAACACGCGCCGTCAAGTTCCGTGGCTTCCCCAGCTGTAGTTGTTTCTGCGGGTGAAGGCGGGCGTGGTAATCTTGCGGTCGAAAT TTGGGACACGCGCCTACGAAGAAGGAAGCCTTCAGTTGTTGCTGAGTGGGGGCCCCCAGTGGGTACGGTGCGCGTGGAATCTGGCGG CGTACAGAAGGTTTACGTAACTGATAACGGACGTGACGGTTTGACAGTTGGTGACATGAGGAAAGTCAGTATGCCGTTAGGAAACGT AACGGAAGTTGACGCGTATAATACTTGGTGGGATGCGGATGCTGTTGTTGTTTCGGATGAATCTTAA

$>$ Contig2747

ATGGCGGAGAAAGAAAATTTCAACGGCAACGGCGTCTACGTGGAACAACAACAACAAGAAGAAGTGGATAAGTTGAAGCACAGAAA AGGTACGTCAGTAAAACAGACCTTGCCTGAGGTCCTTAACAGTCTCGCTTCCGCCATACTCTTTCCGGAACCGGGAGATGACAATAAC TTACTTCGCCGGATCAAAAATTCCGTCGCCGACAACGCTTCACTTCTTCCAGAAGCTTCTAGAAATTCTGCACGTGATATTCTTCTATG GACTCGTCGCGGTAGCCACCTACGCGCTATCCTCGTTATCTCCGTTGGGGCGGTTACTTTTGTTTCTCTTACAGGATTGCTTGTATTTAC GCTTTTCTTTCTGGCCGCAACCATAAATGCCATTGTCATATCGCTGCTGATGTCATTGGCTGCAGCTGGAGGGTTCTTGGCTCTTTTCTT TGCTTGTGCTACCGCTATATACATTGGAGCACTATCAATAGCCATATTTGCAATTTCAGTTACTACATTTTGGACAATTGTGGCTATTC TGATAACCTCAGGGTGGATTGGATTCTTCTATACTGTGTGGCTGATAACTAGAGCGAGTTTCGGGTTTACTAAACACTCATTGAGCGC AACTGGCTCGGCAATCACAACTTACTCTACTTCTCGGGCATCCCGCCAT

$>$ Contig2761

ATGAGAGTGGATATGGAGGTGGACGCTCTACCTATGGAGAAGAAAACCGTTCGGGTGGTTATGGGGACGGAGGGCGTTCAGGTGGTG GCTATGGTGATGAACATTCTAGAAGTGGTTATGGGAATGAACGCGAAGAACGTTTGGGTGGAGGGTATGGTAGGGATAGTCGTGATA ATCGATCCAGTGGTGGATATGGCTATGGAGATAACTAGATGA

$>$ Contig2782

ATGGCTTCAGATGAAGACTTTGAAGATATTATAGATGATGGATTTGATCATAATCATAATCCTAATCCTAATCATCATCTTCAACATCA CCAAAACTTGTCAAGACTTTCAGTTTGTACAAGCAGCACTTTATGTGATGATATTGATAATCTAACTTCCATGTGTATCTCACACTTGT CCATTGAAAGTATGGAAGGTGAAGATGCAGATGCTGAATTCTCAGATGGAAAAGACTTATTATTACAAACAGGGTTATCCTCTGCTTC TGAAAATGACTCCGGTAGTTGTTATTCGTTACCGGCGACACCACCACGACGAAGGAACAGGACTCAAATATCGCCGATTGTAGTGAA AGAGTATGCAA

$>$ Contig2840

ATGGCAGACATCCAGAGAAACCGTCCCGGCGTCACCAGCCACCGCAAGCCAAGTCGATTGCAAAGCCGCGCTCCGTCGTCACTTCAG ATTAACCGTACCGTCGATTGGAACGCTGCGATTCCCCTTCTCTCGCCGCTGGCTTCGTCACCGCCGCCGCCTCCACTACCACAAGCAA AAAGGGAAGAGTCTACGCCGCCGCAGCAGCGCCGGCAGATCGCGGAGGCGGAAAAGATAGTTTTCAAGAAGTGGCAGCACCCTGCG GCACCGTTCTGCTACGGGACTGCTTCAGTGGTTCCGCGCTTCTTTTAA

$>$ Contig2841

ATGGCGGCGAGTTCTAAAGGCTTCTCAACAACGCAGTTCGATTTCAAGCTAAGAGTAAACCGACTCAATGTTGCACTGTTACCTAACA ATCACTTGCCGGACCTTTTCCGGAGAAAGAGAAGAAAGACGTGTATCGATTTTGAACGAGTTCGACTCTGTAATATCAAATTTCGATG CTGTTGTTCTGGTTCGGTTACTCCGATTCTTAGAACGAGTGGACCTG 
ATGGCGAGCGCGTCCTTTGTCCGGAGACAGGCAACTAGCACAAGCCTAAGGGCATTCCCTAACTCGGGTCAGGCTCTATTCGGTGTGA AAGCTGGACGTGGAAGTCGTGTAACTGCGATGGCCGCTTACAAGATAAAACTCATCACTCCAGAGGGAACTAAAGAGTTTCAATGCC CAGATGAAGAAATCATTCTTGATAAAGCAGAGGAAGAAAACATTGACCTTCCTTACTCATGCAGGGCTGGTGCTTGCTCTTCATGTGT TGGAAAAGTTATAGAAGGGAAAGTTGATCAAACAGATAATAGTTTCCTTGATGATGATCAAATAAATGCTGGGTTTATTCTCACATGT GTTGCTTACCCTCGTTCCGATGTTGTTATTGAGACACACAAGGAGGAAGAACTCTCTTCTTAA

$>$ Contig2863

ATGGCCACTTCTTTCGCACCATTATCCATTTCGGCAGGTGGATCTCATCTGAAGTCACCGGAGCTGTTTTTAACAAAGCGTAATTCATC TGTGGTTGGATCTAAGCTTGCTGTTCAAAGGAAATCAAACCTTGTAACCAGGAGAAGGAACCTTACTTCACCTGTTCGCGCCGAATAT AGTGATAGCAGAGGAGGTGGAGGTGGGGATTTTGTTGCTGGATTTCTTCTGGGTGGTGCTGTTTTCGGAACTCTGGCCTATGTTTTTGC TCCCCAGATCAGAAGATCTCTCCTAAATGAAAATGAATATGGGTTTCGGAAGGCCAGAAGACCAATCTATTACGACGAAGGGCTAGA GGGGACCAGGCAGACCTTGAACGAAAAGATAGGGCAACTAAATTCTGCCATTGATAATATATCTTCACGGCTGAGAGGTAAGAATAA TGTGCCTGCTGCCCCTATAGAAAGTGATCCTGAAGTTGAAGCTACCATGTGA

$>$ Contig2882

ATGGTGCCGCTACTTGTTTTGATCGTTGCACTTTTGATTCCAAACGGAGTGTTGTCAATTCCCTCCACAGTCCCTGCATTCCTTTGGTCA TCTCATTATAACTTGGTCTCAGACAATGGATTGAAGGAGTCCGTTAATTATCAGGTCATTTCTCCAAAAGACCTAGCAAAGTCTGTTTT ATCTGAAGCAGGCTGGTCAAATTTTCTGTGCAAAGGGAAGAAATTTCAGGATCCTCTTGATCTGGCACTTCTTTTTGTTGGTGGAGAG TTACAATCTTCAGATTTAAGCTTGAACAAGCATGCAAACTCAGCTCTTTCAGACTTGCTCAAGGACTCTTTTGTCAGATCCAACACTTC CATGGCATTTCCCTATGTGGCAGCATCAGAGGATGTGAATTTGGAAGACTCATTGGTTTCAGGATTTGCTGAAGCCTGTGGAGACGAT TTAGGAATTGGCAACGTTGCTTTCCTTGGATCTTGCTCCATGG

$>$ Contig2888

ATGGACGCAGCATCGCAGCTTGTCTACCGTGGAATCGATCCCTTACTTTGTTCCTCTTATTCCAACCGCAACAACAACCTCCCTCTCCG TCGCCGTTCAAACCGTGTTTTCGCTGTAGCAACCGATCCCAAACCTGCTCCAGTGACAACCGTCAACGGTTCGTCGTCGAGGTCTCCG CCTATCAAACCTGCCAACGGTGTCTCCCAGAGAATTGGTGATGTTTCGAAGGAGATTAAAAGAGTGAGGGCGCAAATGGAAGAAGAC GAACAATTAGCAACTCTCATGAGAGGACTTCGAGGTCAGAATTTGAAGGATTCGCTCTTTGCTGAGGATGATGTTCAGCTACGTCTTG TTGAG

$>$ Contig2892

ATGGCACCTTCCGTCGTCACCACCAGAGTTGCTCCCGCCGTCATTGTCGGCGGTGGAAGAGTTGGTAAAGCTTTACAAGGTATGGGTG ATGGTCAAGATATTCTTCTTCGACGTGGTGATTCTGTTCCTATTGATTTTCAAGGTCCGATTTTGGTTTGTACTCGTAATGATGATCTTG ATTCTGTTCTTAATTCCACTCCTCTTTCCAGATGGAACGATTTGGTGTTTTTCCAAAATGGAATGTTGGAACCATGGCTTGAGAGTAAA GGTTTGAAGGAAGGGAACCAAGTGTTGGCATATTTTGCTGTGTCAAAACTTGGAGAGTCACCTATTGATG

$>$ Contig2969

ATGAATACAACGACCAAGACCACCGAAGTATCCACAACTATATCGATCAATATATACAGATTTCTCCCTGAGAACATATGGTCCAAC AAGATCGTCGCTTCGGCAACAG

$>$ Contig2969_b

ACAAGGCGGCGGAAACACTTAGGAATGACATTGAATGGTTACGGATAACGACGGGTGTGAGCATGGAGAAAGTTGCTTATGAATAA $>$ Contig2984

ATGGCAAATGCTACTATAAGGCAGCAGAGTGTGTGTTTGAACGTAAGGCTGAGAGGATTGAATTTCCATGTTCCTCCTCAGCCTTCTC AGTACTCCATCATTCCTATTTACCACAACACCCACCATTCTTCAATGCTTTGCACCCGCCTGCATCTCACGGTTGTACCTAAAGCTTCG GCCAGAAGCTTAGGCCCCGCTTCTCCTCCCACCGATGACGATGGAGTCTCTTTGGGTACCATGAAGCTTCCTGTCAACATAGACCTTC AGAGATTCGATTCCCTCCTGTTTCAGTGGGCAAACAGCCTTTGCCAGGGAGCCAATCTCCCACTTCCTATGCCTCTCAAGGTGGACAA AATAGCAGGTGGAGCAAGATTAGGATTTATCACTATTGGAGATGGGGAAACAGAAGTTCTTGTGTATATTGATTGTTTGGTTTTTAAA GCCACTGAAAATTCTGCTCCAATATTCCGTGCCTCTAGACATGGACCCTTAAAAGATAAGGTGCCACCTGGTGAGCCCAGAATCATGA GGAGCCTTATGCAAGCTCTTCAGAAATCAGTTCAAATCTCCACATTGTGA

$>$ Contig2989

ATGGGAGGTAGTTTGGATATTCTTGATGAATCTGATAAAGGGGTCTTTTCAAGCCTTGCTCATGGTGTAGCTAATGCTGCTACACATG GAGGAGGACATGGTTATCCACCTGGGGCATACCCTCCACAACAAGGATACCCTCCACAACAAGGTTATCCACCATCTGGGTATCCTCC ACAACAAGGATACCCTCCACAAGGCTACCCACCTGCTGGTTATCCTGCTTCATCTGGTCATAATGCTCAAGGGTCTCATGGACATGGC GGTCCCGGTATGGGAGCAATGCTTGCCGGGGGTGCAGCTGCCGCCGCCGCTGCTTATGGTGCTCACCATATCTCCCACGGCTCTCAAG GTCACTATCCACAAGGTGGATACGCACCAGGTGGCTATGCACAAGGTGGATATGCACATGGTGGTGGTCACATGCCTCACGGAAAAT TCAAGCAGCATGGCAAGTTCAAGCAGGGAAAGCATGGCAAGTTCAAGCATGGAAAGTTTGGCAAGCATGGTGGAGGCAAGCATGGG TTCAAGAAGTGGAAGTGA

$>$ Contig3039

ATGATGCCAAGGAGAGCGGGGAATCCTTCTAGAAGGTTCGGTGATAGTAAATCGAAGACTTCGCCAGTTTTGTCAATTGGGCTTATAG TTGTGGGAGGTTTGTTTCTCATTGGTTACTTGTATAGGGGCTCAGGTGGACATGGAAGCCGTATAGATTCTGTTAGCAAGGTTGAAGG TGATTATTTGTGCAGTGGAGAGGTCCAACGTGCAATTCCTACTTTACAGAAAACATATGGAGATAGCATGCATAAAGTTTTGCATGTT GGTCCTGATACTTGCTATGTGGTCTCTAAATTGAAAAGGGAGGATGAAACTGAAGCCTGGGGTGTAGAACCATATGATATTGAGGAT GTCGATAGTAGTTGCAAATCACTAATCCGTAGAGGCAGTGTGCGTGTGGCTGATGTCAAGTTTCCTCTTCCATATAGGCCAAAATCTT TCTCTCTTGTAATTGTTTCAGATACTCTGGATTACCTATCCCCCAGATACCTCAATAAAACTCTTCCAGATTTGGTGAGGGTATCATCT GATGGTTTAGTGATATTCACTGGTATTCCAACCAATCAAAAGGCCAAGGTAGCAGATGTTTCTAAATTTGGAAGAGCGGCCAAGATG AGGAGTTCATCCTGGTGGGTCAAGTTTTTCCTTCAAAATAACTTAGAGGAGAATGAAGCTGTTAATAAGAAGTTTGAGGAGGCTTCAA CAAAGAGTTCATATGTTCCACGATGCCAGATATTCCACTTGAAGTCACTCCACTGA

$>$ Contig3 103

ATGGGAGGTGGTAAGGATAAGCATGATGAATCTGACAAAGGGGTTTTTTCAAGCCTTGCTCATGGTGTAGCTAATGCTGCTACACATG GAGGAGGACATGGTTATCCACCTGGGGCATACCCTCCACAACATGGGTATCCTCCACAACAAGGATACCCTCCACAACAAGGTTATC CACCATCTGGGTATCCTCCACAACAAGGATACCCTCCACAAGGCTACCCACCTGCTGGTTATCCTGCTTCATCTGGTCATAATGCTCA AGGGTCTCATGGACATGGCGGTCCCGGTATGGGAGCAATGCTTGCCGGGGGTGCAGCTGCCGCCGCCGCTGCTTATGGTGCTCACCAT ATCTCCCACGGCTCTCAAGGTCACTATCCACAAGGTGGATACGCACCAGGTGGCTATGCACAAGGTGGATATGCACATGGTGGTGGT 
CACATGCCTCACGGAAAATTCAAGCAGCATGGCAAGTTCAAGCAGGGAGAGCATGGCAAGTTCAACCATGGAAAGTTTGGCAAGCAT GGTGGAGGCAAGCATGG

$>$ Contig3115

GATTCGAATCCACTCTCACATGTCCCTCCTATCATCACTTATCCACCTAAACTCACTTCCAAATTCTCCTCATCTTCAACACCACAAAA CTTGCATGGCATCTTCACCAAAAGTAAAAACAAGTTCTTGTTGAATGCTGGTTTCAATTTAGTTGAGCCTGACCTCAATGAAGATCCT AGAGATCAATTTAGAACTAATGGCATTGAACCTGAAGAATTTGAGTATGGAATATTTGATGGTCACCACACTTTCAATGAAGGAGAA GAAAAGAAAGGAACATTTTGGGGTGCAATTAGGGATGAGATAGCAGCAGCAGAACCCCCCACTGGATTTCAAGGGCTTATTTCATGG CTCTTCCCACCAGCCATTGCTGCTGGGGTATTCTTCAATGTTCCGGGGGAGTACTTGTACATTGGAGCAGGAATATTTACAATCGTATT TTGCATAATTGAAATGGATAAACCTGATCAACCTCACCACTTTGAACCTCAAATATACAATATGGAAAGAGGAGCTAGAGACAAGTT GATAAATGAATATAATACCATGAGCATATGGGATTTCAATGAAAAATATGGAGACGTTTGGGAATTTACAATCAAGAAGGATGACAT AACCAAAAGATAA

$>$ Contig3121

CTCAGTGATTTTCATGGCGACGCGCAAGATTTGCAGATCCACTGCGTTTCGAAGCACGAGAGGTTCTCTAACTTTAGCAAGCAGAAGT CACACCTTTATCTTTACTGCCGTTCTCTCTCATCCTTGTCTTCTCATAATACTCACCATTCTCTCCTCTACACACTCAACAAAGTCGCCA TTCTCTCTGCACTGTGGTTAACCACTTTGAGCAGTCCTCAAACAAAAAATGACCGCAGGAATCTGATAGAGCCATAG

Supplementary Table S3. Contig wise transcription factors for biotic stresses (wilt, blight) and abiotic stresses (salinity and drought)

\section{Wilt Disease}

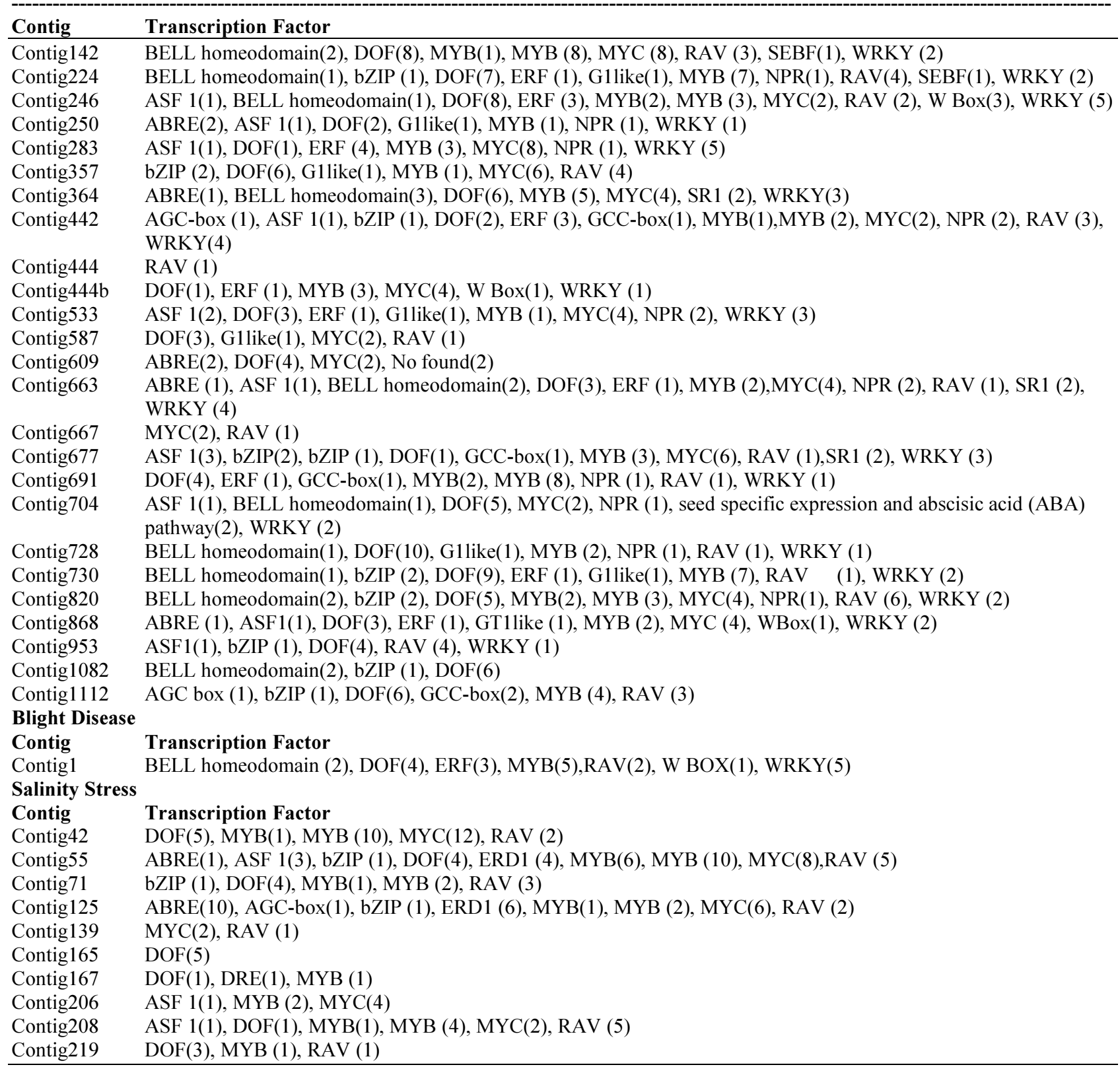




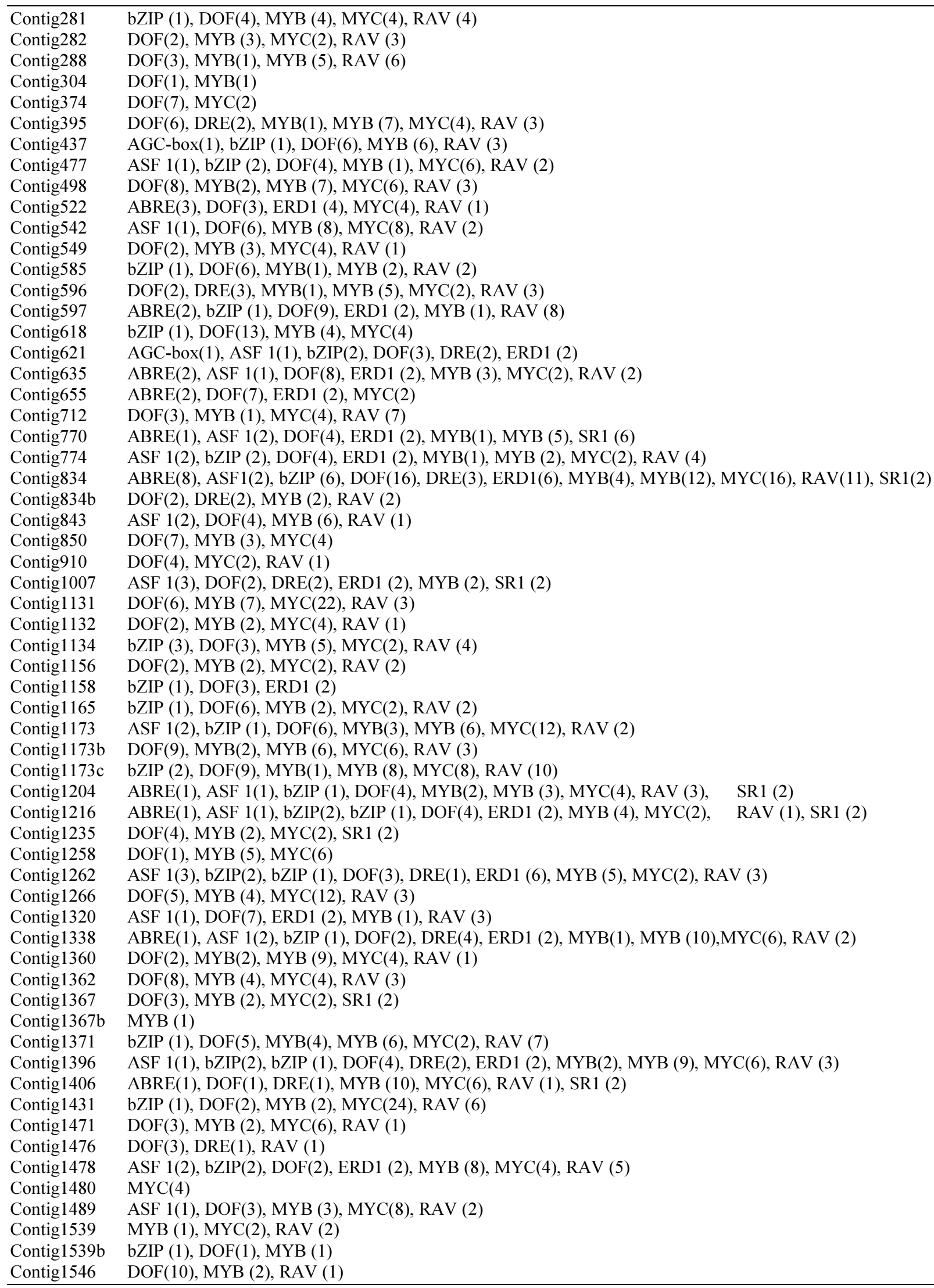

Contig281

Contig282

Contig288

Contig304

Contig374

Contig395

Contig437

Contig477

Contig498

Contig522

Contig542

Contig549

Contig585

Contig596

Contig597

Contig618

Contig621

Contig635

Contig655

Contig712

Contig770

Contig774

Contig834

Contig834b

Contig843

Contig850

Contig910

Contig1007

Contig1131

Contig1132

Contig1134

Contig1156

Contig1158

Contig1165

Contig1173

Contig1173b

Contig1173c

Contig1204

Contig1216

Contig1235

Contig1258

Contig1262

Contig1266

Contig1320

Contig1338

Contig1360

Contig1362

Contig1367

Contig1367b

Contig1371

Contig1396

Contig1406

Contig1431

Contig1471

Contig1476

Contig1478

Contig1480

Contig1489

Contig1539

Contig1539b

Contig1546

bZIP (1), DOF(4), MYB (4), MYC(4), RAV (4)

$\operatorname{DOF}(2), \operatorname{MYB}(3), \operatorname{MYC}(2), \mathrm{RAV}(3)$

DOF(3), MYB(1), MYB (5), RAV (6)

$\operatorname{DOF}(1), \operatorname{MYB}(1)$

$\operatorname{DOF}(7), \operatorname{MYC}(2)$

DOF(6), DRE(2), MYB(1), MYB (7), MYC(4), RAV (3)

AGC-box(1), bZIP (1), DOF(6), MYB (6), RAV (3)

ASF 1(1), bZIP (2), DOF(4), MYB (1), MYC(6), RAV (2)

$\operatorname{DOF}(8), \operatorname{MYB}(2), \operatorname{MYB}(7), \operatorname{MYC}(6), \operatorname{RAV}(3)$

ABRE(3), DOF(3), ERD1 (4), MYC(4), RAV (1)

ASF 1(1), DOF(6), MYB (8), MYC(8), RAV (2)

$\operatorname{DOF}(2), \operatorname{MYB}(3), \operatorname{MYC}(4), \operatorname{RAV}(1)$

bZIP (1), DOF(6), MYB(1), MYB (2), RAV (2)

$\operatorname{DOF}(2), \operatorname{DRE}(3), \operatorname{MYB}(1), \operatorname{MYB}(5), \operatorname{MYC}(2), \operatorname{RAV}(3)$

ABRE(2), bZIP (1), DOF(9), ERD1 (2), MYB (1), RAV (8)

bZIP (1), DOF(13), MYB (4), MYC(4)

AGC-box(1), ASF 1(1), bZIP(2), DOF(3), DRE(2), ERD1 (2)

ABRE(2), ASF 1(1), DOF(8), ERD1 (2), MYB (3), MYC(2), RAV (2)

$\operatorname{ABRE}(2), \operatorname{DOF}(7), \operatorname{ERD} 1(2), \operatorname{MYC}(2)$

$\operatorname{DOF}(3), \operatorname{MYB}(1), \operatorname{MYC}(4), \operatorname{RAV}(7)$

ABRE(1), ASF 1(2), DOF(4), ERD1 (2), MYB(1), MYB (5), SR1 (6)

ASF 1(2), bZIP (2), DOF(4), ERD1 (2), MYB(1), MYB (2), MYC(2), RAV (4)

ABRE(8), ASF1(2), bZIP (6), DOF(16), DRE(3), ERD1(6), MYB(4), MYB(12), MYC(16), RAV(11), SR1(2)

DOF(2), DRE(2), MYB (2), RAV (2)

ASF 1(2), DOF(4), MYB (6), RAV (1)

$\operatorname{DOF}(7)$, MYB (3), MYC(4)

$\operatorname{DOF}(4), \operatorname{MYC}(2)$, RAV (1)

ASF 1(3), DOF(2), DRE(2), ERD1 (2), MYB (2), SR1 (2)

DOF(6), MYB (7), MYC(22), RAV (3)

$\operatorname{DOF}(2), \operatorname{MYB}(2), \operatorname{MYC}(4), \operatorname{RAV}(1)$

bZIP (3), DOF(3), MYB (5), MYC(2), RAV (4)

$\operatorname{DOF}(2), \operatorname{MYB}(2), \operatorname{MYC}(2), \operatorname{RAV}(2)$

bZIP (1), DOF(3), ERD1 (2)

bZIP (1), DOF(6), MYB (2), MYC(2), RAV (2)

ASF 1(2), bZIP (1), DOF(6), MYB(3), MYB (6), MYC(12), RAV (2)

$\operatorname{DOF}(9), \operatorname{MYB}(2), \operatorname{MYB}(6), \operatorname{MYC}(6), \operatorname{RAV}(3)$

bZIP (2), DOF(9), MYB(1), MYB (8), MYC(8), RAV (10)

ABRE(1), ASF 1(1), bZIP (1), DOF(4), MYB(2), MYB (3), MYC(4), RAV (3), $\quad$ SR1 (2)

ABRE(1), ASF 1(1), bZIP(2), bZIP (1), DOF(4), ERD1 (2), MYB (4), MYC(2), RAV (1), SR1 (2)

$\operatorname{DOF}(4)$, MYB (2), MYC(2), SR1 (2)

$\operatorname{DOF}(1)$, MYB (5), MYC(6)

ASF 1(3), bZIP(2), bZIP (1), DOF(3), DRE(1), ERD1 (6), MYB (5), MYC(2), RAV (3)

$\operatorname{DOF}(5)$, MYB (4), MYC(12), RAV (3)

ASF 1(1), DOF(7), ERD1 (2), MYB (1), RAV (3)

ABRE(1), ASF 1(2), bZIP (1), DOF(2), DRE(4), ERD1 (2), MYB(1), MYB (10),MYC(6), RAV (2)

$\operatorname{DOF}(2), \operatorname{MYB}(2), \operatorname{MYB}(9), \operatorname{MYC}(4), \operatorname{RAV}(1)$

$\operatorname{DOF}(8)$, MYB (4), MYC(4), RAV (3)

$\operatorname{DOF}(3)$, MYB (2), MYC(2), SR1 (2)

MYB (1)

bZIP (1), DOF(5), MYB(4), MYB (6), MYC(2), RAV (7)

ASF 1(1), bZIP(2), bZIP (1), DOF(4), DRE(2), ERD1 (2), MYB(2), MYB (9), MYC(6), RAV (3)

ABRE(1), DOF(1), DRE(1), MYB (10), MYC(6), RAV (1), SR1 (2)

bZIP (1), DOF(2), MYB (2), MYC(24), RAV (6)

DOF(3), MYB (2), MYC(6), RAV (1)

DOF(3), DRE(1), RAV (1)

ASF 1(2), bZIP(2), DOF(2), ERD1 (2), MYB (8), MYC(4), RAV (5)

MYC(4)

$\operatorname{ASF} 1(1), \operatorname{DOF}(3), \operatorname{MYB}(3), \operatorname{MYC}(8), \operatorname{RAV}(2)$

MYB (1), MYC(2), RAV (2)

bZIP (1), DOF(1), MYB (1)

DOF(10), MYB (2), RAV (1) 


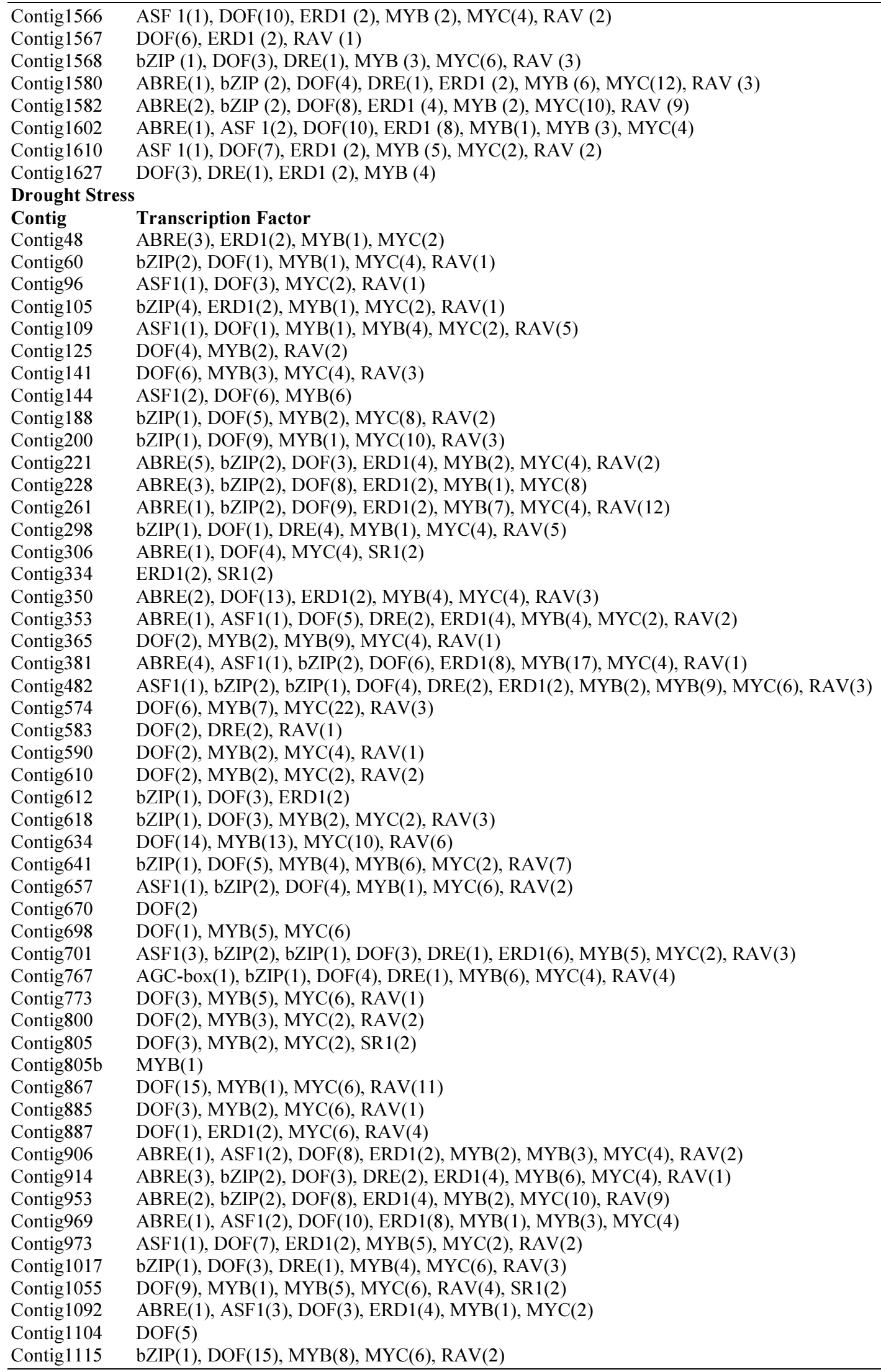

Transcription Factor

ABRE(3), ERD1(2), MYB(1), MYC(2)

bZIP(2), DOF(1), MYB(1), MYC(4), RAV(1)

$\operatorname{ASF} 1(1), \operatorname{DOF}(3), \operatorname{MYC}(2), \operatorname{RAV}(1)$

bZIP(4), ERD1(2), MYB(1), MYC(2), RAV(1)

ASF1(1), DOF(1), MYB(1), MYB(4), MYC(2), RAV(5)

$\operatorname{DOF}(4), \operatorname{MYB}(2), \operatorname{RAV}(2)$

$\operatorname{DOF}(6), \operatorname{MYB}(3), \operatorname{MYC}(4), \operatorname{RAV}(3)$

$\operatorname{ASF} 1(2), \operatorname{DOF}(6), \operatorname{MYB}(6)$

bZIP(1), DOF(5), MYB(2), MYC(8), RAV(2)

bZIP(1), DOF(9), MYB(1), MYC(10), RAV(3)

ABRE(5), bZIP(2), DOF(3), ERD1(4), MYB(2), MYC(4), RAV(2)

ABRE(3), bZIP(2), DOF(8), ERD1(2), MYB(1), MYC(8)

$\operatorname{ABRE}(1), \operatorname{bZIP}(2), \operatorname{DOF}(9), \operatorname{ERD} 1(2), \operatorname{MYB}(7), \operatorname{MYC}(4), \operatorname{RAV}(12)$

bZIP(1), DOF(1), DRE(4), MYB(1), MYC(4), RAV(5)

ABRE(1), DOF(4), MYC(4), SR1(2)

ERD1(2), SR1(2)

ABRE(2), DOF(13), ERD1(2), MYB(4), MYC(4), RAV(3)

$\operatorname{ABRE}(1), \operatorname{ASF} 1(1), \operatorname{DOF}(5), \operatorname{DRE}(2), \operatorname{ERD1}(4), \operatorname{MYB}(4), \operatorname{MYC}(2), \operatorname{RAV}(2)$

$\operatorname{DOF}(2), \operatorname{MYB}(2), \operatorname{MYB}(9), \operatorname{MYC}(4), \operatorname{RAV}(1)$

ABRE(4), ASF1(1), bZIP(2), DOF(6), ERD1(8), MYB(17), MYC(4), RAV(1)

ASF1(1), bZIP(2), bZIP(1), DOF(4), DRE(2), ERD1(2), MYB(2), MYB(9), MYC(6), RAV(3)

$\operatorname{DOF}(6), \operatorname{MYB}(7), \operatorname{MYC}(22), \operatorname{RAV}(3)$

$\operatorname{DOF}(2), \operatorname{DRE}(2), \operatorname{RAV}(1)$

$\operatorname{DOF}(2), \operatorname{MYB}(2), \operatorname{MYC}(4), \operatorname{RAV}(1)$

$\operatorname{DOF}(2), \operatorname{MYB}(2), \operatorname{MYC}(2), \operatorname{RAV}(2)$

bZIP(1), DOF(3), ERD1(2)

bZIP(1), DOF(3), MYB(2), MYC(2), RAV(3)

$\operatorname{DOF}(14), \operatorname{MYB}(13), \operatorname{MYC}(10), \operatorname{RAV}(6)$

bZIP(1), DOF(5), MYB(4), MYB(6), MYC(2), RAV(7)

$\operatorname{ASF} 1(1), \operatorname{bZIP}(2), \operatorname{DOF}(4), \operatorname{MYB}(1), \operatorname{MYC}(6), \operatorname{RAV}(2)$

$\operatorname{DOF}(2)$

$\operatorname{DOF}(1), \operatorname{MYB}(5), \operatorname{MYC}(6)$

ASF1(3), bZIP(2), bZIP(1), DOF(3), DRE(1), ERD1(6), MYB(5), MYC(2), RAV(3)

AGC-box(1), bZIP(1), DOF(4), DRE(1), MYB(6), MYC(4), RAV(4)

$\operatorname{DOF}(3), \operatorname{MYB}(5), \operatorname{MYC}(6), \operatorname{RAV}(1)$

$\operatorname{DOF}(2), \operatorname{MYB}(3), \operatorname{MYC}(2), \operatorname{RAV}(2)$

$\operatorname{DOF}(3), \operatorname{MYB}(2), \operatorname{MYC}(2), \operatorname{SR} 1(2)$

$\operatorname{MYB}(1)$

$\operatorname{DOF}(15), \operatorname{MYB}(1), \operatorname{MYC}(6), \operatorname{RAV}(11)$

$\operatorname{DOF}(3), \operatorname{MYB}(2), \operatorname{MYC}(6), \operatorname{RAV}(1)$

$\operatorname{DOF}(1), \operatorname{ERD1}(2), \operatorname{MYC}(6), \operatorname{RAV}(4)$

ABRE(1), ASF1(2), DOF(8), ERD1(2), MYB(2), MYB(3), MYC(4), RAV(2)

$\operatorname{ABRE}(3), b Z I P(2), \operatorname{DOF}(3), \operatorname{DRE}(2), \operatorname{ERD1}(4), \operatorname{MYB}(6), \operatorname{MYC}(4), \operatorname{RAV}(1)$

$\operatorname{ABRE}(2), \operatorname{bZIP}(2), \operatorname{DOF}(8), \operatorname{ERD} 1(4), \operatorname{MYB}(2), \operatorname{MYC}(10), \operatorname{RAV}(9)$

ABRE(1), ASF1(2), DOF(10), ERD1(8), MYB(1), MYB(3), MYC(4)

$\operatorname{ASF} 1(1), \operatorname{DOF}(7), \operatorname{ERD} 1(2), \operatorname{MYB}(5), \operatorname{MYC}(2), \operatorname{RAV}(2)$

bZIP(1), DOF(3), DRE(1), MYB(4), MYC(6), RAV(3)

$\operatorname{DOF}(9), \operatorname{MYB}(1), \operatorname{MYB}(5), \operatorname{MYC}(6), \operatorname{RAV}(4), \operatorname{SR} 1(2)$

ABRE(1), ASF1(3), DOF(3), ERD1(4), MYB(1), MYC(2)

$\operatorname{DOF}(5)$

bZIP(1), DOF(15), MYB(8), MYC(6), RAV(2) 


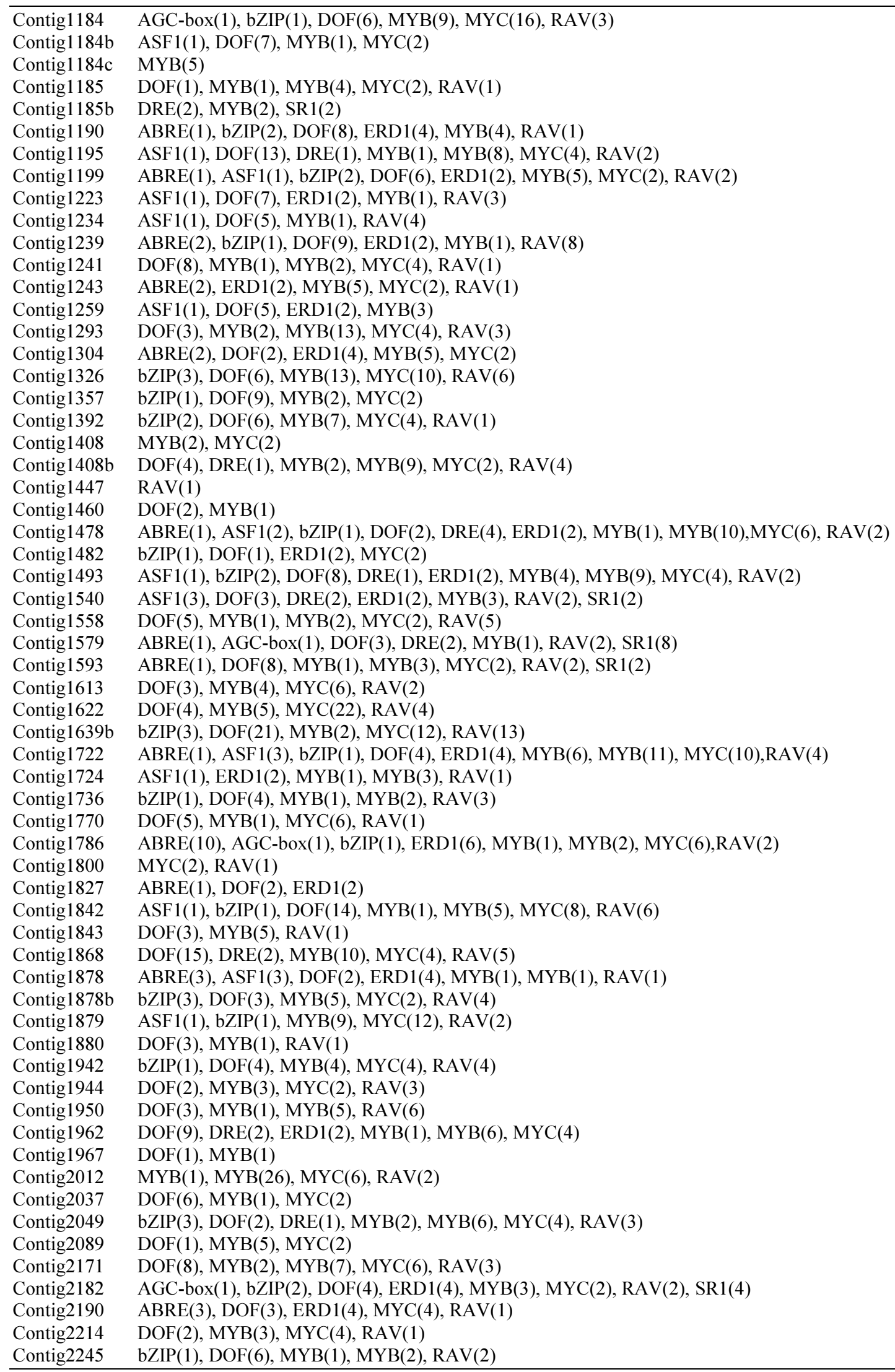

Contig1184

Contig1184b

Contig1184c

Contig1185

Contig1185b

Contig1190

Contig1195

Contig1199

Contig1223

Contig1234

Contig1239

Contig1241

Contig1243

Contig1259

Contig1293

Contig1304

Contig1326

Contig1357

Contig1392

Contig1408

Contig1408b

Contig1447

Contig1460

Contig1478

Contig1482

Contig1493

Contig1540

Contig1558

Contig1579

Contig1593

Contig1613

Contig1622

Contig1639b

Contig1722

Contig1724

Contig1736

Contig1770

Contig1786

Contig1800

Contig1827

Contig1842

Contig1843

Contig1868

Contig1878

Contig1878b

Contig1879

Contig1880

Contig1942

Contig1944

Contig1950

Contig1962

Contig1967

Contig2012

Contig2037

Contig2049

Contig2089

Contig2171

Contig2182

Contig2190

Contig2214

Contig2245

AGC-box(1), bZIP(1), DOF(6), MYB(9), MYC(16), RAV(3)

$\operatorname{ASF} 1(1), \operatorname{DOF}(7), \operatorname{MYB}(1), \operatorname{MYC}(2)$

MYB(5)

$\operatorname{DOF}(1), \operatorname{MYB}(1), \operatorname{MYB}(4), \operatorname{MYC}(2), \operatorname{RAV}(1)$

$\operatorname{DRE}(2), \operatorname{MYB}(2), \operatorname{SR} 1(2)$

ABRE(1), bZIP(2), DOF(8), ERD1(4), MYB(4), RAV(1)

$\operatorname{ASF} 1(1), \operatorname{DOF}(13), \operatorname{DRE}(1), \operatorname{MYB}(1), \operatorname{MYB}(8), \operatorname{MYC}(4), \operatorname{RAV}(2)$

ABRE(1), ASF1(1), bZIP(2), DOF(6), ERD1(2), MYB(5), MYC(2), RAV(2)

ASF1(1), DOF(7), ERD1(2), MYB(1), RAV(3)

$\operatorname{ASF} 1(1), \operatorname{DOF}(5), \operatorname{MYB}(1), \operatorname{RAV}(4)$

ABRE(2), bZIP(1), DOF(9), ERD1(2), MYB(1), RAV(8)

$\operatorname{DOF}(8), \operatorname{MYB}(1), \operatorname{MYB}(2), \operatorname{MYC}(4), \operatorname{RAV}(1)$

ABRE(2), ERD1(2), MYB(5), MYC(2), RAV(1)

$\operatorname{ASF} 1(1), \operatorname{DOF}(5), \operatorname{ERD1}(2), \operatorname{MYB}(3)$

$\operatorname{DOF}(3), \operatorname{MYB}(2), \operatorname{MYB}(13), \operatorname{MYC}(4), \operatorname{RAV}(3)$

$\operatorname{ABRE}(2), \operatorname{DOF}(2), \operatorname{ERD} 1(4), \operatorname{MYB}(5), \operatorname{MYC}(2)$

bZIP(3), DOF(6), MYB(13), MYC(10), RAV(6)

bZIP(1), DOF(9), MYB(2), MYC(2)

bZIP(2), DOF(6), MYB(7), MYC(4), RAV(1)

$\operatorname{MYB}(2), \operatorname{MYC}(2)$

$\operatorname{DOF}(4), \operatorname{DRE}(1), \operatorname{MYB}(2), \operatorname{MYB}(9), \operatorname{MYC}(2), \operatorname{RAV}(4)$

RAV(1)

$\operatorname{DOF}(2), \operatorname{MYB}(1)$

ABRE(1), ASF1(2), bZIP(1), DOF(2), DRE(4), ERD1(2), MYB(1), MYB(10),MYC(6), RAV(2)

bZIP(1), DOF(1), ERD1(2), MYC(2)

ASF1(1), bZIP(2), DOF(8), DRE(1), ERD1(2), MYB(4), MYB(9), MYC(4), RAV(2)

ASF1(3), DOF(3), DRE(2), ERD1(2), MYB(3), RAV(2), SR1(2)

$\operatorname{DOF}(5), \operatorname{MYB}(1), \operatorname{MYB}(2), \operatorname{MYC}(2), \operatorname{RAV}(5)$

ABRE(1), AGC-box(1), DOF(3), DRE(2), MYB(1), RAV(2), SR1(8)

ABRE(1), DOF(8), MYB(1), MYB(3), MYC(2), RAV(2), SR1(2)

$\operatorname{DOF}(3), \operatorname{MYB}(4), \operatorname{MYC}(6), \operatorname{RAV}(2)$

$\operatorname{DOF}(4), \operatorname{MYB}(5), \operatorname{MYC}(22), \operatorname{RAV}(4)$

bZIP(3), DOF(21), MYB(2), MYC(12), RAV(13)

ABRE(1), ASF1(3), bZIP(1), DOF(4), ERD1(4), MYB(6), MYB(11), MYC(10), RAV(4)

$\operatorname{ASF} 1(1), \operatorname{ERD1}(2), \operatorname{MYB}(1), \operatorname{MYB}(3), \operatorname{RAV}(1)$

bZIP(1), DOF(4), MYB(1), MYB(2), RAV(3)

$\operatorname{DOF}(5), \operatorname{MYB}(1), \operatorname{MYC}(6), \operatorname{RAV}(1)$

ABRE(10), AGC-box(1), bZIP(1), ERD1(6), MYB(1), MYB(2), MYC(6),RAV(2)

$\operatorname{MYC}(2), \operatorname{RAV}(1)$

$\operatorname{ABRE}(1), \operatorname{DOF}(2), \operatorname{ERD1}(2)$

ASF1(1), bZIP(1), DOF(14), MYB(1), MYB(5), MYC(8), RAV(6)

$\operatorname{DOF}(3), \operatorname{MYB}(5), \operatorname{RAV}(1)$

$\operatorname{DOF}(15), \operatorname{DRE}(2), \operatorname{MYB}(10), \operatorname{MYC}(4), \operatorname{RAV}(5)$

$\operatorname{ABRE}(3), \operatorname{ASF} 1(3), \operatorname{DOF}(2), \operatorname{ERD1}(4), \operatorname{MYB}(1), \operatorname{MYB}(1), \operatorname{RAV}(1)$

bZIP(3), DOF(3), MYB(5), MYC(2), RAV(4)

$\operatorname{ASF} 1(1), \operatorname{bZIP}(1), \operatorname{MYB}(9), \operatorname{MYC}(12), \operatorname{RAV}(2)$

$\operatorname{DOF}(3), \operatorname{MYB}(1), \operatorname{RAV}(1)$

bZIP(1), DOF(4), MYB(4), MYC(4), RAV(4)

$\operatorname{DOF}(2), \operatorname{MYB}(3), \operatorname{MYC}(2), \operatorname{RAV}(3)$

$\operatorname{DOF}(3), \operatorname{MYB}(1), \operatorname{MYB}(5), \operatorname{RAV}(6)$

$\operatorname{DOF}(9), \operatorname{DRE}(2), \operatorname{ERD1}(2), \operatorname{MYB}(1), \operatorname{MYB}(6), \operatorname{MYC}(4)$

$\operatorname{DOF}(1), \operatorname{MYB}(1)$

$\operatorname{MYB}(1), \operatorname{MYB}(26), \operatorname{MYC}(6), \operatorname{RAV}(2)$

$\operatorname{DOF}(6), \operatorname{MYB}(1), \operatorname{MYC}(2)$

bZIP(3), DOF(2), DRE(1), MYB(2), MYB(6), MYC(4), RAV(3)

$\operatorname{DOF}(1), \operatorname{MYB}(5), \operatorname{MYC}(2)$

$\operatorname{DOF}(8), \operatorname{MYB}(2), \operatorname{MYB}(7), \operatorname{MYC}(6), \operatorname{RAV}(3)$

AGC-box(1), bZIP(2), DOF(4), ERD1(4), MYB(3), MYC(2), RAV(2), SR1(4)

$\operatorname{ABRE}(3), \operatorname{DOF}(3), \operatorname{ERD1}(4), \operatorname{MYC}(4), \operatorname{RAV}(1)$

$\operatorname{DOF}(2), \operatorname{MYB}(3), \operatorname{MYC}(4), \operatorname{RAV}(1)$

bZIP(1), DOF(6), MYB(1), MYB(2), RAV(2) 


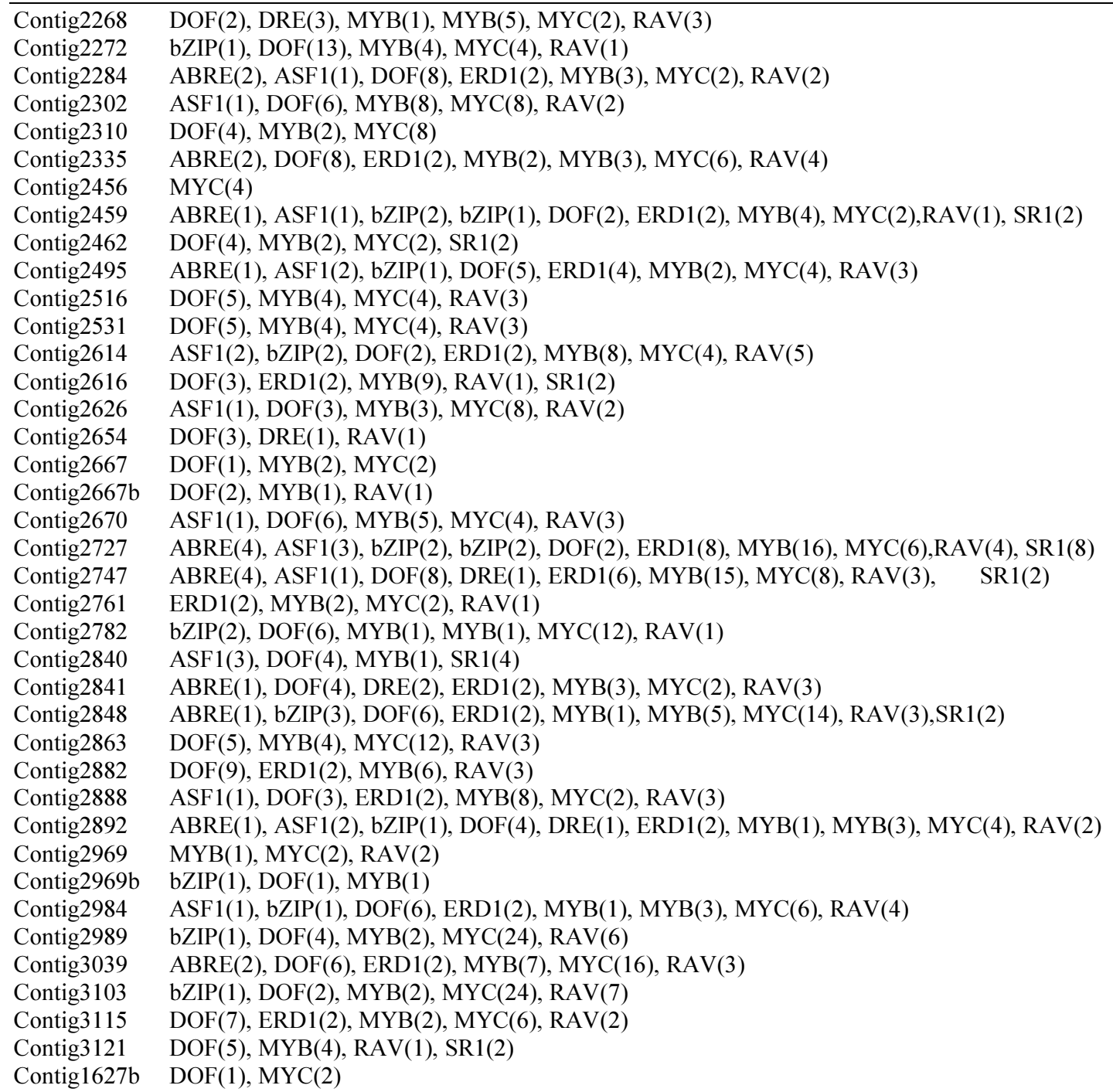

